\title{
SYNTHETIC APERTURE RADAR RAPID DETECTION OF RANGE AND AZIMUTH VELOCITIES IMPLEMENTED IN MATLAB
}

\author{
A Thesis \\ presented to \\ the Faculty of the California Polytechnic State University,
}

San Luis Obispo

In Partial Fulfillment

of the Requirements for the Degree

Master of Science in Electrical Engineering

by

Cheuk Yu David So

June 2013 
(C) 2013

Cheuk Yu David So

ALL RIGHTS RESERVED 


\section{COMMITTEE MEMBERSHIP}

TITLE:

AUTHOR:

DATE SUBMITTED: June 2013

COMMITTEE CHAIR: Dr. John A. Saghri, Professor, Electrical Engineering

COMMITTEE MEMBER: Dr. Jane Zhang, Associate Professor, Electrical Engineering

COMMITTEE MEMBER: Dr. John Y. Oliver, Associate Professor, Electrical Engineering 


\begin{abstract}
Synthetic Aperture Radar Rapid Detection of Range and Azimuth Velocities Implemented in MATLAB

Cheuk Yu David So
\end{abstract}

The Synthetic Aperture Radar (SAR) algorithm processes multiple radar returns from the target space to generate a single high-resolution image. Targets moving through the target space during the capture sequence will appear distorted on the final image. In addition, there is no velocity information that is calculated as part of the processing. The objective of this thesis is to develop techniques to determine the azimuth and range velocities of moving objects in the target space in the early stages of SAR processing. The typical SAR processing steps are Range Compressed, Range Doppler, and final image generation. The range velocity of a target can be determined after the Range Compression stage, and the azimuth velocity can be determined after the Range Doppler image is created. Calculating the velocity of a target without performing all the steps of the SAR process allows such information can be obtained quicker than the final image.

This work is done as part of Cal Poly's SAR Automatic Target Recognition (ATR) project, sponsored by Raytheon Space and Airborne Systems Division and headed by Professor John Saghri. The simulations performed as part of this thesis are done in a MATLAB simulation environment implementing a two-dimension SAR target space, first introduced in Brian Zaharris' thesis. This work has expanded on this environment by introducing point target azimuth and range velocity detection. 


\section{ACKNOWLEDGMENTS}

I would like to thank my faculty advisor, Dr. John Saghri, for his continual support and guidance during the research and preparation of this thesis. His guidance proved instrumental in my thesis work. I would also like to thank Raytheon for its sponsorship of my thesis along with the rest of the Synthetic Aperture Radar research at Cal Poly. Their support has offered many students the opportunity to explore this exciting field, as the subject of their theses and senior projects. I would like to thank Brian Zaharris and Cindy Romero for their research in this field. Their work proved very helpful during my own research in Synthetic Aperture Radar, and provided the background for my thesis. I am also grateful to my parents for their unwavering support and encouragement while I was working on my thesis. Their support and guidance have allowed me to pursue a Bachelor's and Master's degree in Electrical Engineering. 


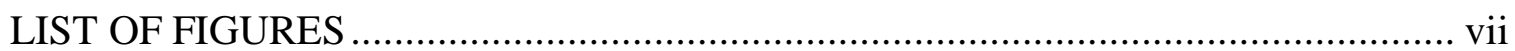

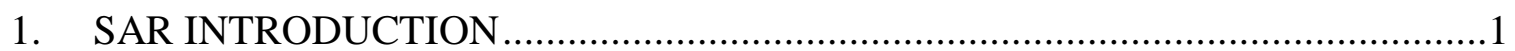

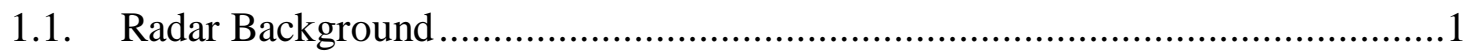

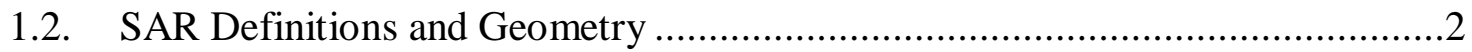

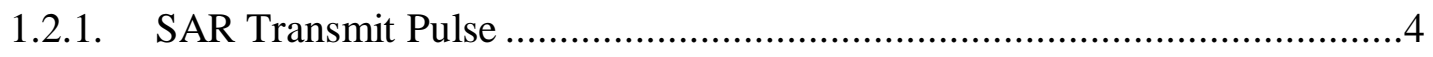

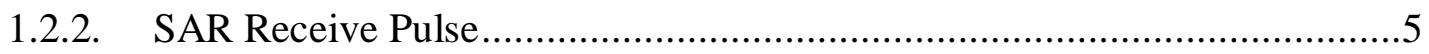

1.3. The Range Doppler Algorithm.............................................................11

1.3.1. Range Compressed Signal ................................................................12

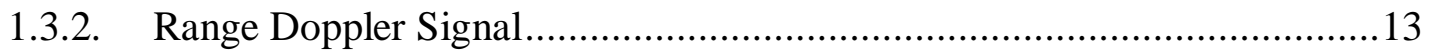

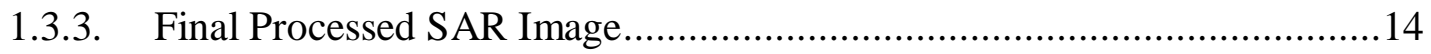

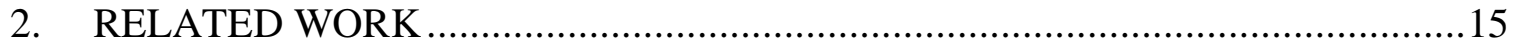

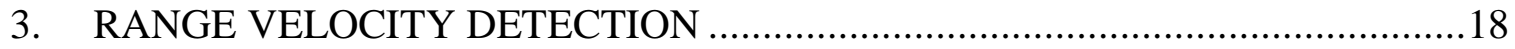

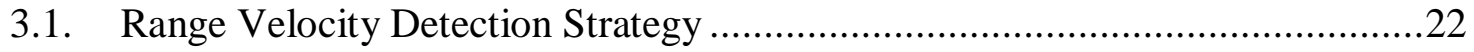

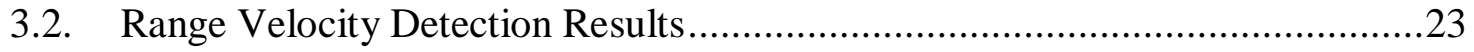

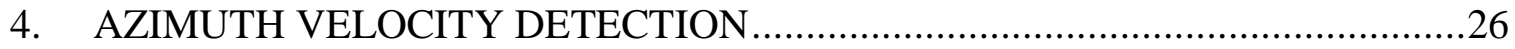

4.1. Azimuth Velocity Detection in the Range-Compressed Space ........................29

4.2. Azimuth Velocity Detection in the Range-Doppler Space ..................................36

4.3. Range-Compressed Azimuth Velocity Detection Results ..................................40

4.4. Range-Doppler Azimuth Velocity Detection Results .......................................49

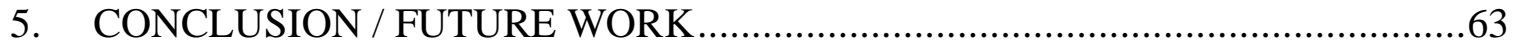

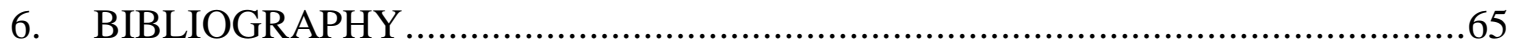

APPENDIX A: MATLAB CODE USER GUIDE...................................................67 


\section{TABLE OF FIGURES}

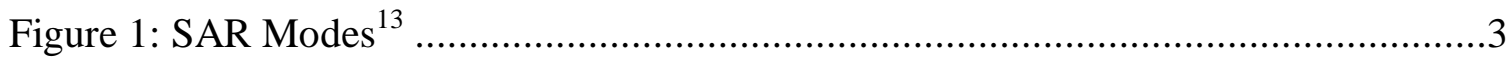

Figure 2: Effect of Pulse Repetition Interval on Transmit and Receive Pulse ${ }^{13}$.................5

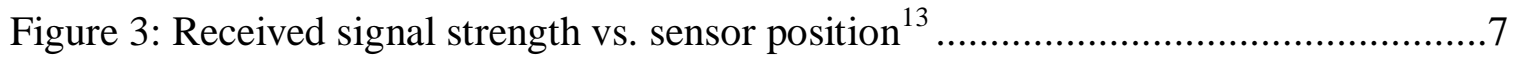

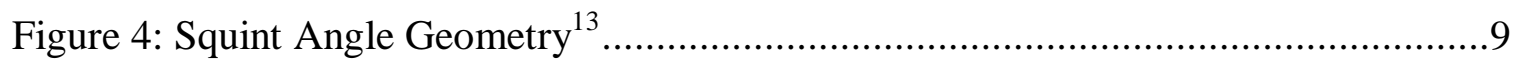

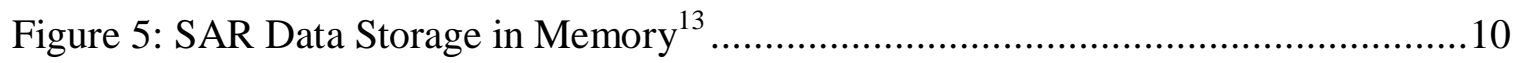

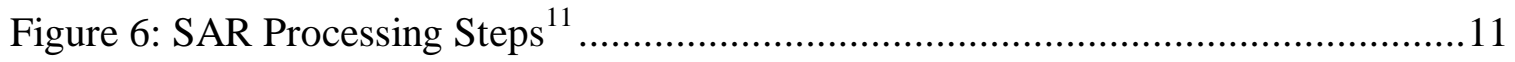

Figure 7: Matched Filter Output ${ }^{12}$.....................................................................12

Figure 8: Range Compressed Signal at Varying Range Velocities ..................................18

Figure 9: Azimuth Fourier Transform Signal at Varying Range Velocities.....................19

Figure 10: Final Target Signal at Varying Range Velocities ........................................21

Figure 11: Measured vs. Actual Range Velocity of Target .............................................23

Figure 12: Percent Error of Range Velocity Calculations ...........................................24

Figure 13: Range Compressed Signal at Varying Azimuth Velocities ...........................26

Figure 14: Azimuth Fourier Transform Signal at Varying Azimuth Velocities ...............27

Figure 15: Final Target Imagining Signal at Varying Azimuth Velocities .....................28

Figure 16: Expanded View of Range Compressed Signal.............................................30

Figure 17: Range Compressed Signal vs. Azimuth Bin ................................................31

Figure 18: Range Compressed Signal vs. Azimuth Bin, With Positive Velocity ..............32

Figure 19: Range Compressed Signal vs. Azimuth Bin, With Negative Velocity.............33

Figure 20: Measured Peak Distance vs. Target Azimuth Velocity ...................................34

Figure 21: Target Azimuth Velocity vs. Measured Peak Distance ....................................35 
Figure 22: Range-Doppler Plot of Targets With Azimuth Velocities .38

Figure 23: Magnified Range-Doppler Plot of Targets With Azimuth Velocities .39

Figure 24: Measured vs. Actual Azimuth Velocities of Targets.................................41

Figure 25: Absolute Error of Range-Compressed Azimuth Velocity Calculations ..........42

Figure 26: Percent Error of Range-Compressed Azimuth Velocity Calculations ............43

Figure 27: Range Compressed Signal vs. Azimuth Bin, no AWGN............................44

Figure 28: Range Compressed Signal vs. Azimuth Bin, AWGN added .......................45

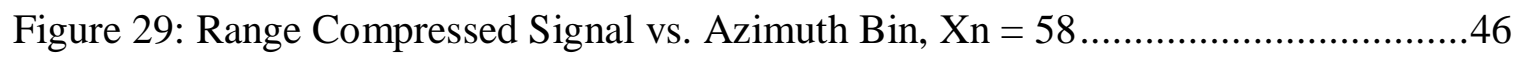

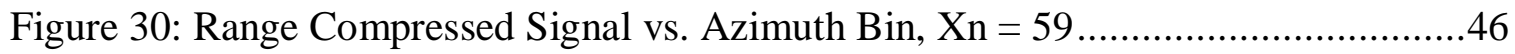

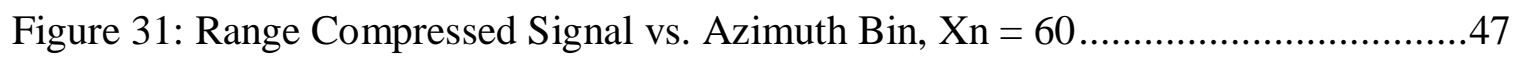

Figure 32: Measured Peak Distances vs. Starting Range Positions of Target .................48

Figure 33: Range-Doppler Peak Values vs. Target Azimuth Velocities .........................50

Figure 34: Target Azimuth Velocities vs. Range-Doppler Peak Values ........................51

Figure 35: Range-Doppler Peak Values at Various Starting Range Positions ................52

Figure 36: Signal Variance of Target With Azimuth Velocity of $-30 \mathrm{~m} / \mathrm{s}$....................53

Figure 37: Signal Variance of Target With Azimuth Velocity of $0 \mathrm{~m} / \mathrm{s}$.......................54

Figure 38: Signal Variance of Target With Azimuth Velocity of $+30 \mathrm{~m} / \mathrm{s}$....................54

Figure 39: Signal Variance vs. Azimuth Velocity of Target Located At Xn $=0$...........55

Figure 40: Azimuth Velocity vs. Signal Variance of Target Located At Xn $=0$..........56

Figure 41: Signal Variance vs. Azimuth Velocity of Target Located At Xn $=1 \ldots \ldots \ldots . .57$

Figure 42: Azimuth Velocity vs. Signal Variance of Target Located At Xn $=1 \ldots \ldots \ldots . .57$

Figure 43: Signal Variance vs. Azimuth Velocity of Target Located At Xn $=2 \ldots \ldots \ldots . .58$

Figure 44: Azimuth Velocity vs. Signal Variance of Target Located At Xn $=2$...........59 
Figure 45: Absolute Error of Azimuth Velocities of Target Located at $\mathrm{Xn}=0 \ldots \ldots \ldots \ldots . .60$

Figure 46: Percent Error of Azimuth Velocities of Target Located at Xn $=0$..............60

Figure 47: Absolute Error of Azimuth Velocities of Target Located at Xn $=1 \ldots \ldots \ldots \ldots . .61$

Figure 48: Percent Error of Azimuth Velocities of Target Located at Xn $=1 \ldots \ldots \ldots \ldots \ldots . .61$

Figure 49: Absolute Error of Azimuth Velocities of Target Located at Xn $=2 \ldots \ldots \ldots \ldots . .62$

Figure 50: Percent Error of Azimuth Velocities of Target Located at Xn $=2$.............62 


\section{SAR INTRODUCTION}

\subsection{Radar Background}

Radio Detection and Ranging (RADAR or radar) is a detection system that is used in many civilian and military applications. The radar system composed of a transmitter and receiver. It operates by periodically transmitting electromagnetic waves towards an area or object of interest. The reflected waves are captured by the receiver, which then analyzes the returning waveform to determine properties of the object, such as distance, heading, and velocity. The distance to the object is most simply calculated by determining the time between the wave's propagation from the transmitter to its detection at the receiver. The transmitter in a radar system allows for detection without relying on an external source to illuminate the target space. This is an advantage over other detection systems, since illumination from the sun or other light source is not necessary to identify or track the target [8].

Due to these advantages, radar is used in many remote sensing applications in the civilian and military sectors [3]. The Synthetic Aperture Radar (SAR) technique is often employed to increase the resolution of radar systems. When mounted on a moving platform, the limited length of the transmitter antenna that can be mounted on an aircraft reduces the performance of these systems. Under the SAR process, multiple pulse returns are collected and stored as the platform moves across the target space. SAR exploits the known location of each pulse, assuming the radar platform is moving at a constant velocity. By extracting the range and azimuth spectral information from the collected 
returned pulses, a much higher resolution can be achieved compared with traditional processing algorithms.

\subsection{SAR Definitions and Geometry}

There are two directions of interest when discussing the behavior of Synthetic Aperture Radar: azimuth and range. The azimuth direction is parallel with the motion of the radar platform, and is also known as the "slow time" direction. This is due to the relatively slow velocity of the platform relative to the radar pulses. The range direction is oriented orthogonal to the platform motion, and parallel to the beam direction. This direction is also referred to as the "fast time", as this is the direction that the radar pulses are transmitted.

As the platforms moves in the azimuth direction, multiple radar pulses are sent along the range direction. For each radar pulse sent out, a certain area in the target space is illuminated and contributes to the radar return. This area is known as the footprint, and is the part of the target space that radiates signal back to the receiver. In the "stripmap" mode of SAR, the pulses are always orthogonal to the direction of travel. Subsequently, the radar pulses cover each area in the target space for an equal amount of time.

Alternatively, the radar can be configured to point towards a certain area continuously as the platform moves across the target space. This technique is called "spotlight mode", analogous to a spotlight being focused at a target [1]. Since the radar is continuously focused on one location instead of sweeping a target area orthogonally, spotlight mode results in more radar returns from the target in question, but at the expense of the total area covered by the radar returns. The simulation environment used for this thesis uses the stripmap SAR mode. The two modes are shown in Figure 1. 


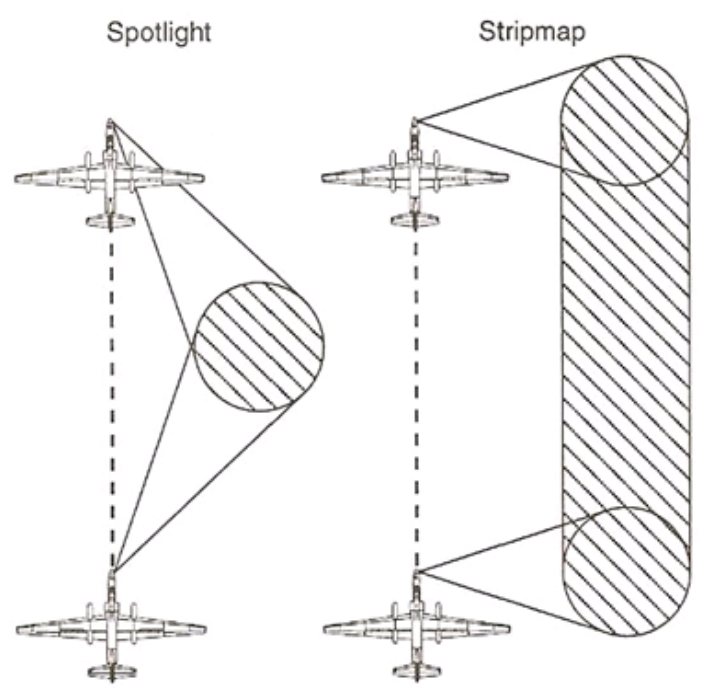

Figure 1: SAR Modes ${ }^{13}$

As the platform moves across the target space, the distance between the radar transmitter and the target of interest continually changes. This distance is called the slant range, and is an important parameter for calculating the Doppler shift the radar pulses experience at each transmission location. The squint angle is closely related, which is the angle that the slant range makes with the zero Doppler shift plane; that is, the range that results in no Doppler shift. The squint angle and slant range are important parameters in the calculations for the Doppler shift of the platform that are used to combine the multiple radar returns into a single image.

In addition to distance variables, there are time variables that are used in SAR calculations. "Fast time" is the time variable for the speed of the radar pulses. "Slow time" is the time variable used for the velocity of the platform. These variables will be used later in the derivation of the SAR signal. 
The SAR pulsed radar operates on a series of transmit and receive cycles. The echoes received by the radar are aggregated and used to compute the SAR signal space. Each of the two stages will be discussed at length.

\subsubsection{SAR Transmit Pulse}

During SAR radar operation, a frequency-modulated signal is transmitted by the radar. The signal is characterized by Equation 1.

$$
s_{p u l}(t)=w_{r}(t) \cos \left(2 \pi f_{c} t+\pi K_{r} t^{2}\right)
$$

\section{Equation 1}

$w_{r}(t)=$ Rectangular function representing pulse duration in fast time

$f_{c}=$ Carrier frequency

$K_{r}=$ Chirp Rate

The $\pi K_{r} t^{2}$ term introduces a chirp in the transmitted signal, which causes a linear change in frequency as a function of fast time. A positive value of $\mathrm{K}_{\mathrm{r}}$ is referred to an "up chirp", while a negative value is referred to as a "down chirp". The inclusion of an up chirp or a down chirp improves the accuracy of the range information, since the received frequency can be used to determine exactly when the pulse reached the target. The individual parameters will be discussed further in subsequent sections.

Other important values used in SAR are the signal bandwidth, $B_{0}$, chirp pulse duration, $t_{r}$, and the range resolution, $\rho_{\mathrm{r}}$. Equations 2 and 3 describe the relationships between the variables. 


$$
B o=\left|K_{r}\right| t_{r}
$$

\section{Equation 2}

$$
\rho_{r}=\frac{\mathrm{c}}{2 B_{o}}
$$

\section{Equation 3}

$c=$ speed of light

Each frequency-modulated signal is transmitted at a specified frequency, known as the Pulse Repetition Frequency (PRF). The inverse of the PRF is called the Pulse Repetition Interval. Figure 2 illustrates the transmit and receive sequence of a SAR signal.

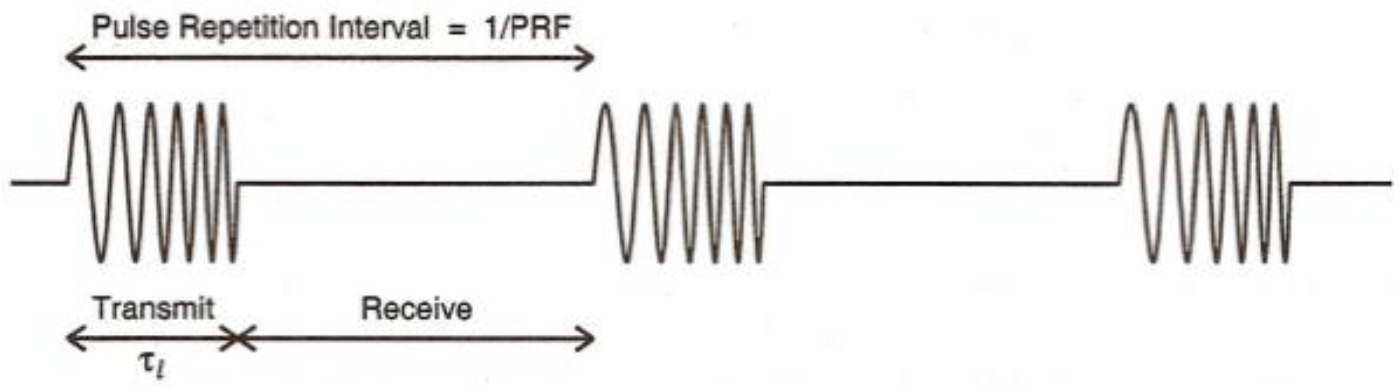

Figure 2: Effect of Pulse Repetition Interval on Transmit and Receive Pulse ${ }^{13}$

As can be seen, there is a linear increase in frequency of the transmit pulse. This is due to an up chirp added to the signal.

\subsubsection{SAR Receive Pulse}

Once the SAR signal has been transmitted, the system listens for the received signal. A single received signal can be represented as shown in Equation 4.

$$
s_{r}(t)=F_{n} * w_{r}\left(t-\frac{2 R_{o}}{c}\right) e^{\left.-j 4 \pi \frac{f_{c} R_{o}}{c}+\pi K_{r}\left(t-\frac{2 R_{o}}{c}\right)^{2}\right)}
$$


$R_{0}=$ range at zero slant angle

\section{Equation 4}

$F_{n}=$ reflectivity of target

$c=$ speed of light

$f_{c}=$ carrier frequency

The received signal is proportional to the reflectivity of the target, as well as the flight time of the signal from the transmitter and then to the receiver. The total time is twice the instantaneous slant range to the target, divided by the speed of light. The received signal is complex and with both a baseband and high frequency carrier terms since the signal has been quadrature modulated. The high frequency carrier term will be removed.

As the radar platform moves through the target space, there are additional factors that need to be considered to characterize the received signal. The movement in the azimuth direction, or slow time, affects both the slant range and the amplitude of the signal.

In this paper, the platform is assumed to be moving at a constant velocity in the azimuth direction, and in azimuth and range distances small enough that there is a clear line of sight from the platform to all points in the target space. The equation for the instantaneous slant range as a function of slow time is shown as Equation 5.

$$
R(\eta)=\sqrt{R_{0}{ }^{2}+V_{r}{ }^{2} \eta^{2}}
$$

$R_{0}=$ range at zero slant angle

\section{Equation 5}

$V_{r}=$ velocity of radar platform

$\eta=$ slow time 
The slow time, $\eta$, is considered to be negative before the platform reaches zero slant angle and positive as the platform moves away. As Figure 3 shows, the distance of closest approach is achieved when the slant angle is zero.

In addition to the slant range, the amplitude of the received signal is also affected by the slow time. As shown in Figure 3, the strongest return occurs when the center of the beam pattern is on the target. The small side lobes also produce small peaks where the return is strong.

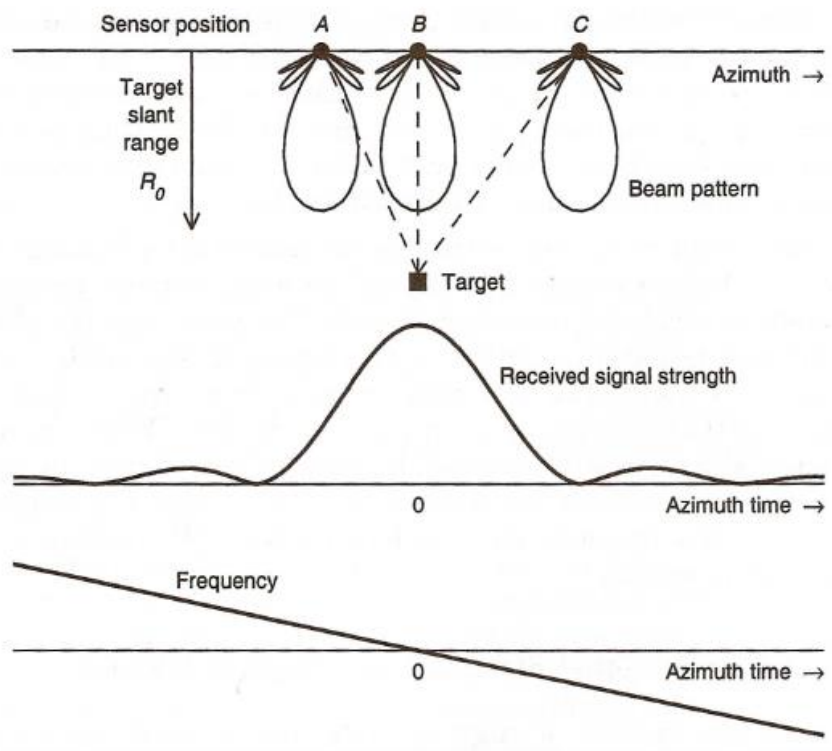

Figure 3: Received signal strength vs. sensor position $^{13}$

When the target first enters the target space, the platform is moving towards the target, and so the Doppler frequency shift is positive. This case is shown in sensor position A, when the target is entering the main lobe of the signal. When the target is situated in the middle of the bin (sensor position B), the relative velocity between the platform and the 
target is zero, so there is no frequency shift. As the platform continues moving, the Doppler shift becomes increasingly negative. This situation is shown in position $\mathrm{C}$. The target has passed from the main lobe of the system. However, the transmitted signal has small side lobes that contribute some energy, so the received signal has small side lobes as well.

Equation 6 shows the amplitude of the received signal as a function of slow time.

$$
\omega_{a}(\eta) \approx \rho_{a}^{2}\{\theta(\eta)\} \approx \operatorname{sinc}^{2}\left(\frac{0.886 \theta(\eta)}{\beta_{b w}}\right)
$$

\section{Equation 6}

Due to the propagation of the signal in both directions, the returning beam pattern is approximated by a $\operatorname{sinc}^{2}$ function, and is expressed as a function of $\eta$.

The variations in the instantaneous slant range and amplitude result in the modified received signal equation, which is shown as Equation 7.

$$
s_{r}(t, \eta)=F_{n} * w_{r}\left(t-\frac{2 R(\eta)}{c}\right) w_{a}\left(\eta-\eta_{c}\right) e^{\left.-j 4 \pi \frac{f_{c} R_{\eta}}{c}+\pi K_{r}\left(t-\frac{2 R_{\eta}}{c}\right)^{2}\right)}
$$

\section{Equation 7}

The equation for the Doppler bandwidth of the SAR system is shown as Equation 8.

$$
\Delta f_{\text {dop }}=\frac{2 V_{s} \cos \theta_{s q}}{\lambda} \theta_{b w}
$$

\section{Equation 8}

The Doppler bandwidth is an important quantity, as is affects the minimum sampling rate needed in the azimuth resolution. According to the Nyquist theorem, the SAR sampling 
rate in the azimuth resolution must meet or exceed the Doppler bandwidth to avoid aliasing of the signal.

The squint angle is represented by $\theta_{s q}$, and is the angle between the zero Doppler plane and the vector from the platform to the target. The squint angle is shown in Figure 4.

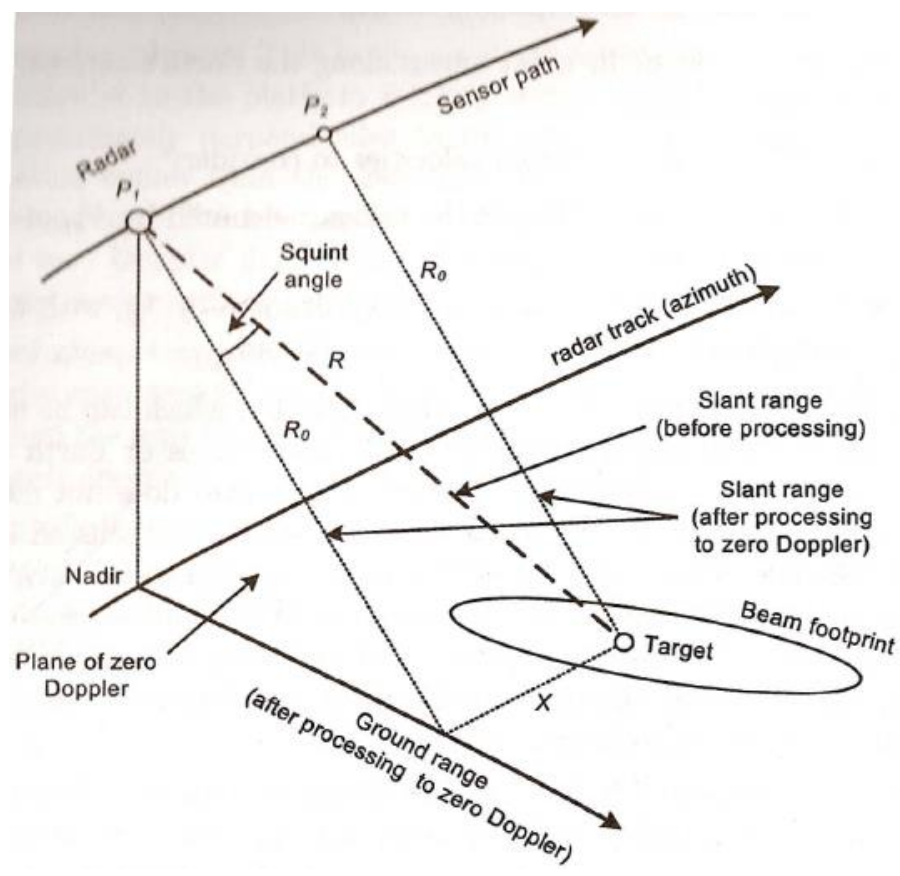

Figure 4: Squint Angle Geometry ${ }^{13}$

The squint angle is a function of the location of the platform, and subsequently is dependent on the azimuth time of the system. The equation for the squint angle is shown as Equation 9.

$$
\theta_{s q}=\arccos \left(\frac{R_{o_{m}}}{R_{m}(\eta)}\right)
$$

\section{Equation 9}

It decreases as the platform approaches the target, reaches zero when the platform is at its closest position to the target, and increases as the platform continues past the target. 
Each of the received signals is stored in a two-dimensional array, as shown in Figure 5.

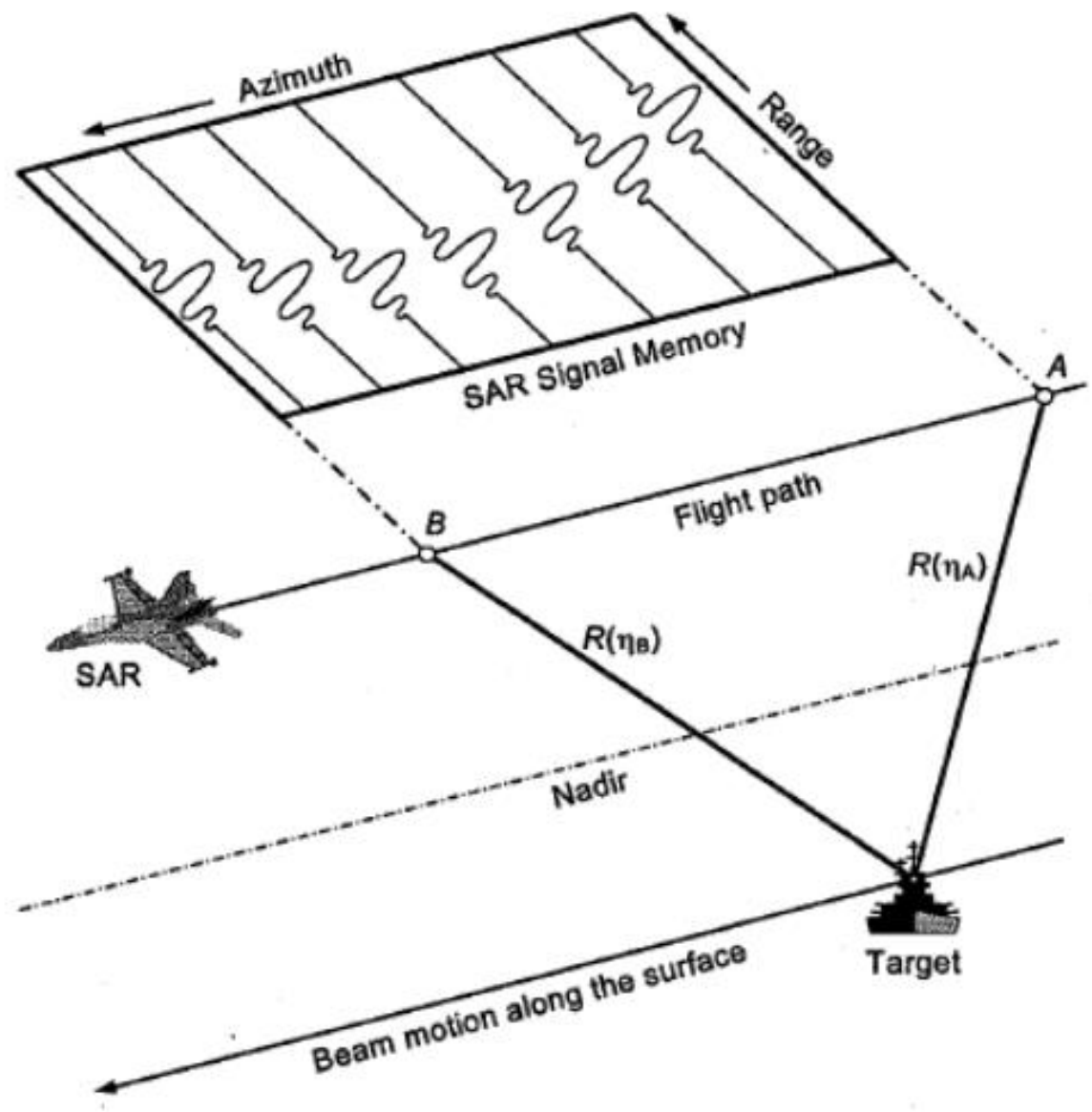

Figure 5: SAR Data Storage in Memory $^{13}$

Each signal is added into the array as it is received, and each point of the signal is stored horizontally. This orders the data such that each row represents the entirety of a single received signal, and each column is the data received from a particular range distance. 


\subsection{The Range Doppler Algorithm}

The Range Doppler algorithm is a widely used Synthetic Aperture Radar processing technique that utilizes match filtering to generate high-resolution images of targets. Range Doppler processing is very efficient because the match filtering process can be done entirely in the frequency domain. The convolution operations that would be necessary in the time domain can therefore be replaced by multiplication operations, greatly increasing processing speed. In addition, the entire target space is processed at once, allowing all targets to be processed simultaneously with a single set of operations. The name "Range Doppler" stems from a particular step in the process, when the target space data in the azimuth, or Doppler, direction is represented in the frequency domain, and the range data has already been compressed. The steps involved in the Range Doppler are detailed in the following sections.

The block diagram for the Range Doppler algorithm is shown as Figure 6.
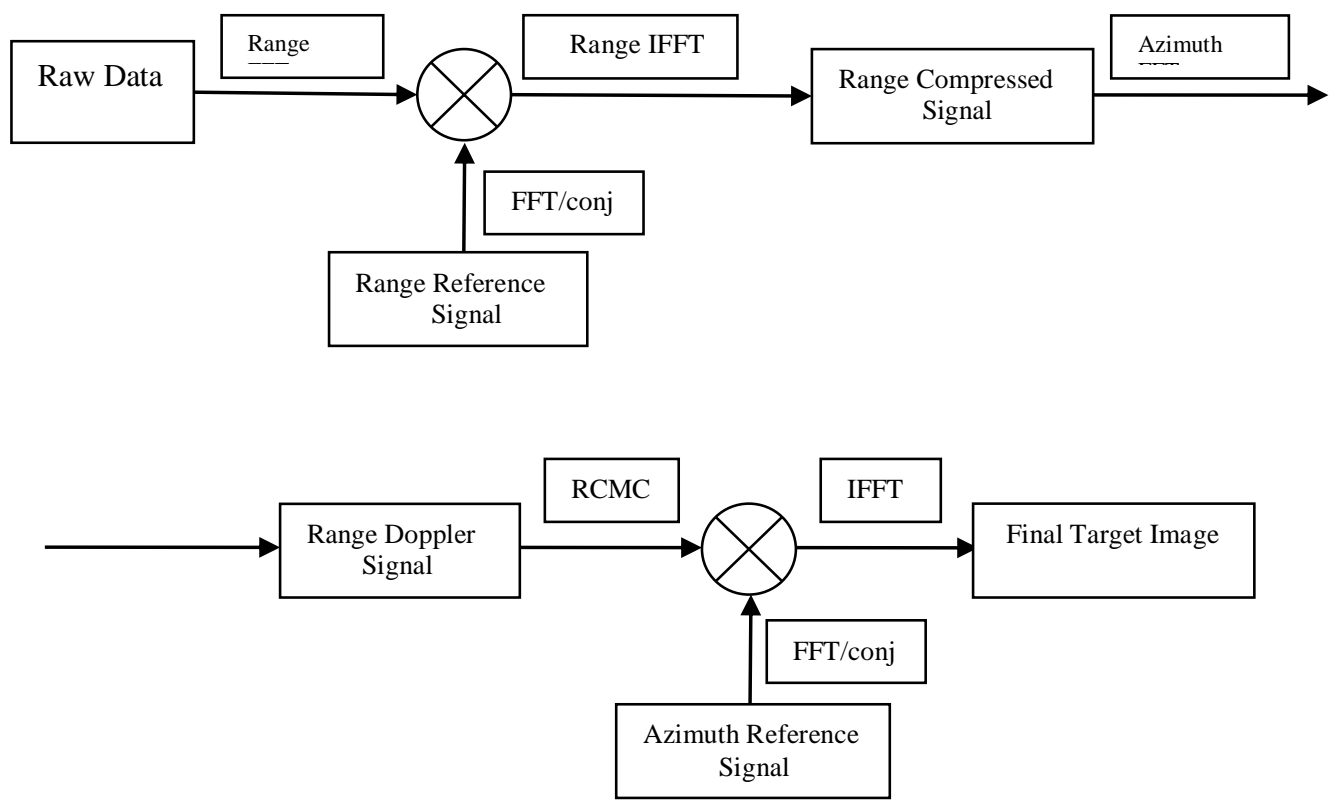

Figure 6: SAR Processing Steps ${ }^{11}$ 
The signals that are fed into the algorithm are the raw SAR signal space, Range

Reference Signal, and Azimuth Reference Signal. The raw SAR signal space is the array of data which is captured by the radar platform as it traverses the target space. The Range and Azimuth Reference Signals are the ideal returns of a single point source. It is used as part of the "matched filtering" process. The operations involved in the process are FFTs, IFFTs, and multiplications of signals.

\subsubsection{Range Compressed Signal}

The first step in the algorithm is to compress the raw data in the range direction. This is done with a process called matched filtering. In the matched filtering process, a reference signal is compared with the incoming raw data to find the location where the received data matches a certain reference signal. In this context, the reference signal refers to the noiseless, ideal return signal of a theoretical point target. The matched filtering process is shown in Figure 7.

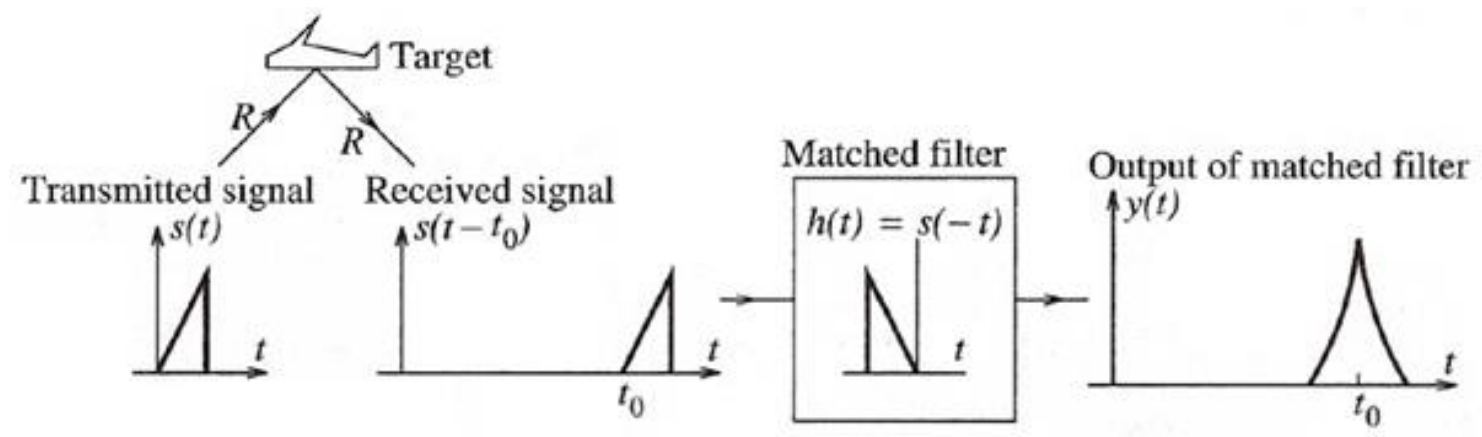

Figure 7: Matched Filter Output ${ }^{12}$

If the matched filtering is done in the time domain, the reference signal would be convolved with the complex conjugate of the received signal, and the peak location of the result would indicate where the raw SAR signal matched the reference signal. In this Range Doppler implementation, a FFT is performed along each range bin of the raw SAR 
signal, as well as to the reference signal. This places both signals in the frequency domain. Once that is done, the match filtering is done with a simple multiplication of the raw SAR signal and reference signal. An inverse FFT is then performed along each range bin of the signal to bring it back to the time domain. The resulting signal is referred to as the "Range Compressed signal".

\subsubsection{Range Doppler Signal}

The Range Doppler signal is obtained from the Range Compressed signal described in section 1.3.1. Once the Range Compressed signal is generated, a second set of FFTs are performed. This time, the FFTs are run with respect to the azimuth direction. The output of the transform will be in the time domain in the range direction, and the frequency domain in the azimuth direction. This signal is known as the "Range Doppler" signal, since the azimuth frequency, or Doppler frequency, is obtained.

The Range Cell Migration Correction (RCMC) is typically applied at this stage. The instantaneous slant range changes as the platform moves through the target space. Because of this, the target has a different Doppler frequency at different times throughout the simulation, and subsequently appears at multiple range bins in the Range Doppler signal. If the signal is compressed, the final image would show a washed out return instead of a point target. To correct for this, Range Cell Migration Correction is applied. The amount of shift, or migration, in meters can be calculated for each point on the Range Doppler signal by Equations 10 and 11. 


$$
R_{r d\left(f_{\eta}\right)}=\frac{R_{o_{m}}}{\sqrt{1-\frac{c^{2} f_{\eta}^{2}}{4 V_{r}^{2} f_{O}^{2}}}} \approx \frac{\lambda^{2} R_{o_{m}} f_{\eta}^{2}}{8 V_{r}^{2}}
$$

\section{Equation 10}

$$
f_{\eta} \approx-K_{a} \eta \approx \frac{2 V_{r}{ }^{2} \eta}{\lambda R_{o_{m}}}
$$

\section{Equation 11}

Since the Range Doppler signal data is quantized into discrete range and azimuth bins, the exact shift may not be able to be represented. Therefore, there will still be a slight blurring of the target in the final image.

\subsubsection{Final Processed SAR Image}

Once the RCMC has been applied to the Range Doppler signal, it is processed into the final image. Similar to the Range Compressed process, the signal is convolved with a reference azimuth signal using matched filtering. Since the Range Doppler signal is already in the frequency domain in the azimuth direction, it is simply multiplied with a reference azimuth signal in the frequency domain as well. The signal is then fed through an inverse FFT in the azimuth direction, resulting in the final target image. 


\section{RELATED WORK}

Determining a moving target's velocity in a SAR target space is a subject of considerable attention. There has been significant research into being able to reliably velocity information of targets in a SAR application, and in SAR target tracking in general.

The standard SAR algorithm was designed for imaging stationary targets in a target space. It relies on known velocity and distances between the platform and the target so that the matched filtering in the azimuth and range directions are appropriate. If the target begins to move while the platform is sampling the target space, the resulting image will show a blurry image of the target spread over multiple range and azimuth bins [1] [4]. The change in Doppler frequency of the received signals causes the target to be distorted [2]. The target's velocity during the sampling sequence is not readily obvious from the SAR final target image. There have been many papers published detailing research towards determining information about the target's velocity and direction.

In Brian Zaharris' thesis, he explored using a Kalman filter to track the changing slant range of a moving target to determine the velocity of the target. The implementation of the Kalman filter that Zaharris used is one-dimensional. For each azimuth and range sample, the amount of movement by the target is calculated by comparing the expected slant range against the measured slant range. This movement distance is fed into the Kalman filter to predict the subsequent movement distances. There is no information on the direction of the target velocity, and the simulation assumes that the point target moves only in the azimuth or range direction. In addition, the Kalman filter requires a certain time for its output to accurately track the target velocity. 
A strategy to detect the movement of targets is presented in "Detecting Moving Targets in SAR Imagery by Focusing" [6]. In this approach, the SAR algorithm is performed in its entirety, resulting in the final SAR image. The image is then segmented into small image patches. Each patch undergoes a FFT in the azimuth direction, and a phase error is calculated. A moving target is considered to be detected when this phase error exceeds a predefined threshold. This approach is very computationally intensive, due to the number of FFTs that need to be performed on the final image. The SAR final image itself is generated after multiple FFTs in both the azimuth and range directions, and there will be a time delay from when the raw data is obtained to when the final image in constructed. Because of this large computational overhead, the target space that can be analyzed with this method is reduced [4]. In addition, no information of the target velocity can be determined.

Another approach, as described in Multiple Target Tracking, is to generate a "Range Time" plot, showing the range of the target as function of time [9]. This presents a visual representation of the movement of the target. The generation of the "Range Time" plot requires additional computation time. Compared to the approach taken in this paper, the Range Time plot is able to display the state of the target for each sample. This allows changes of velocity to be illustrated. However, the azimuth velocity of the target is not calculated.

A technique to calculate the range and azimuth velocities of a target is discussed in "Estimation of Target Movement Parameters in Synthetic Aperture Radar" [6]. The distance from the target to the sensor platform is expressed as a function of the target's azimuth and range velocity, as well as the platform velocity. The received SAR data, 
along with the target distance, is then used to approximate the values of the target velocities by solving the 3D non-linear least squares problem. This technique requires significant computational time to compute a solution. In addition, the implementation laid out in the paper requires multiple antennas and receivers to calculate the target distance at each SAR data point, which is used to solve the least squares problem. The approach taken here utilizes a single transmitter/receiver pair to calculate the target range and azimuth velocities.

The MATLAB experiments performed as part of the thesis is largely based on the target space simulation framework created by Brian Zaharris. His MATLAB model for SAR signals creates a 900x1024 grid that point targets can be placed in. The radar returns from a SAR transmitter/receiver moving at a constant velocity are simulated, and a final target image of the scene is generated by using the SAR algorithm. Additional routines were added onto the module to implement the various velocity detection techniques. The specific parameters used in the target space simulation are detailed in Appendix A. 


\section{RANGE VELOCITY DETECTION}

To determine the behavior of the SAR signal, five targets are placed in the simulation space, with each target moving at a different range velocity. The velocities that were used are $-60,-30,0,+30$, and $+60 \mathrm{~m} / \mathrm{s}$. Each target was spaced evenly, 100 bins apart, on the azimuth axis, so each target's returns will not interfere with the neighboring target. The resulting waveforms were then analyzed at each step of the SAR processing algorithm, to identify any distinguishing behavior that could help determine the velocity for each target.

The first step of the process results in the Range Compressed signal, and the waveform at that stage is shown in Figure 8.

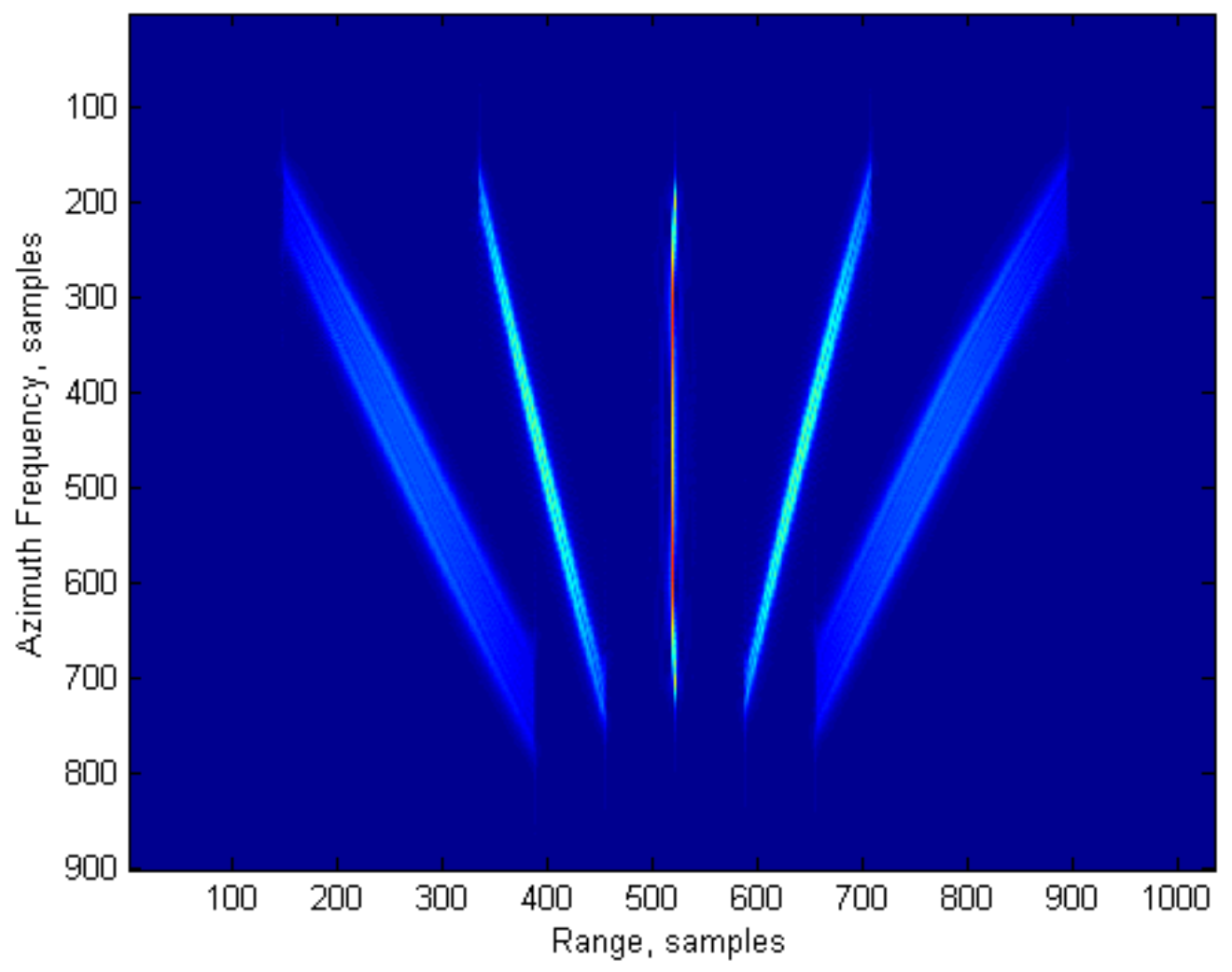

Figure 8: Range Compressed Signal at Varying Range Velocities 
At positive velocities of the target, the range increases as the azimuth increases. Since the targets are moving away from the azimuth axis, the targets are farther and farther away as the platform moves across the target space. Conversely, when the velocity is negative, the range values decrease at increasing azimuth values. At constant velocities, the range values change linearly as expected. The next stage of the process applies a Fourier transform in the azimuth direction, and results in Figure 9.

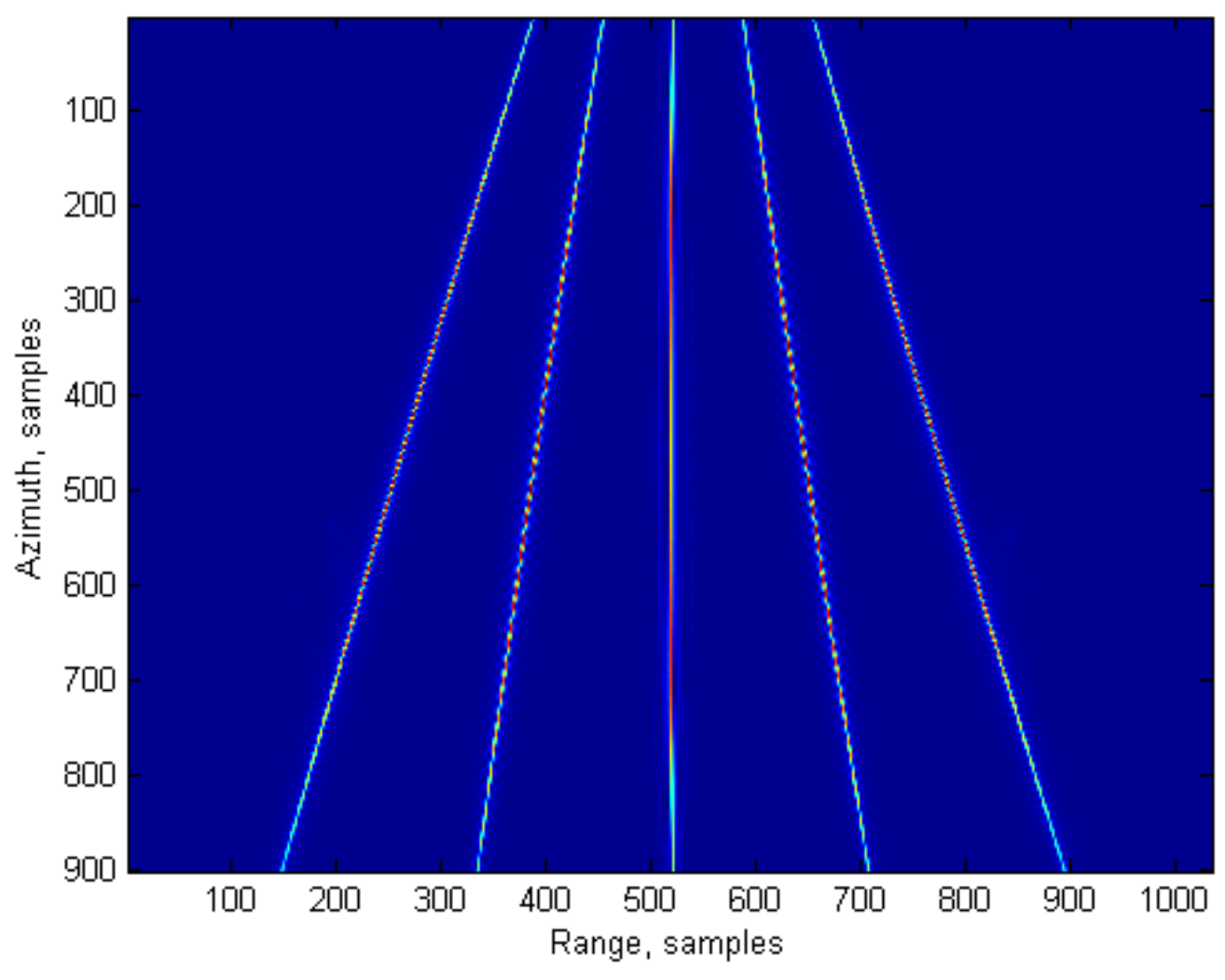

Figure 9: Azimuth Fourier Transform Signal at Varying Range Velocities

The signal at this stage of processing is known as the Range Doppler signal. There is a linear relationship between the velocity of the target and the range values. The difference is that the vertical axis is Azimuth frequency instead of azimuth bins that are filled as the platform transverses the target space. Small azimuth values correspond to negative Doppler shifts, which occur when the radar platform is moving away from the target as it 
leaves the target space. Large azimuth values correspond to positive Doppler shifts at the beginning of the simulation as the platform first enters the target space. Therefore targets with a positive range velocity will have large range values at small azimuth frequencies, and small range values at large frequencies. This behavior is reversed for negative velocities.

Unlike the Range Compressed signals, the waveforms are not a straight line, but with multiple range values occurring at each azimuth frequency. This is due to shape of the return signal - as shown in Figure 3, there are side lobes present that cause a point target to appear over a spread of azimuth values. At a zero range velocity, the spread of azimuth values always present themselves across the same range value, causing the response to look like a single line. As the target moves in the range direction, each spread of azimuth values show up at slightly different ranges each time. A larger absolute velocity causes the signature to appear over more range values, which is reflected in the graph above.

The final target image is shown in Figure 10. In this image, only the three targets with range velocities of $-30,0$, and $+30 \mathrm{~m} / \mathrm{s}$ are shown, to prevent the signatures from overlapping. 


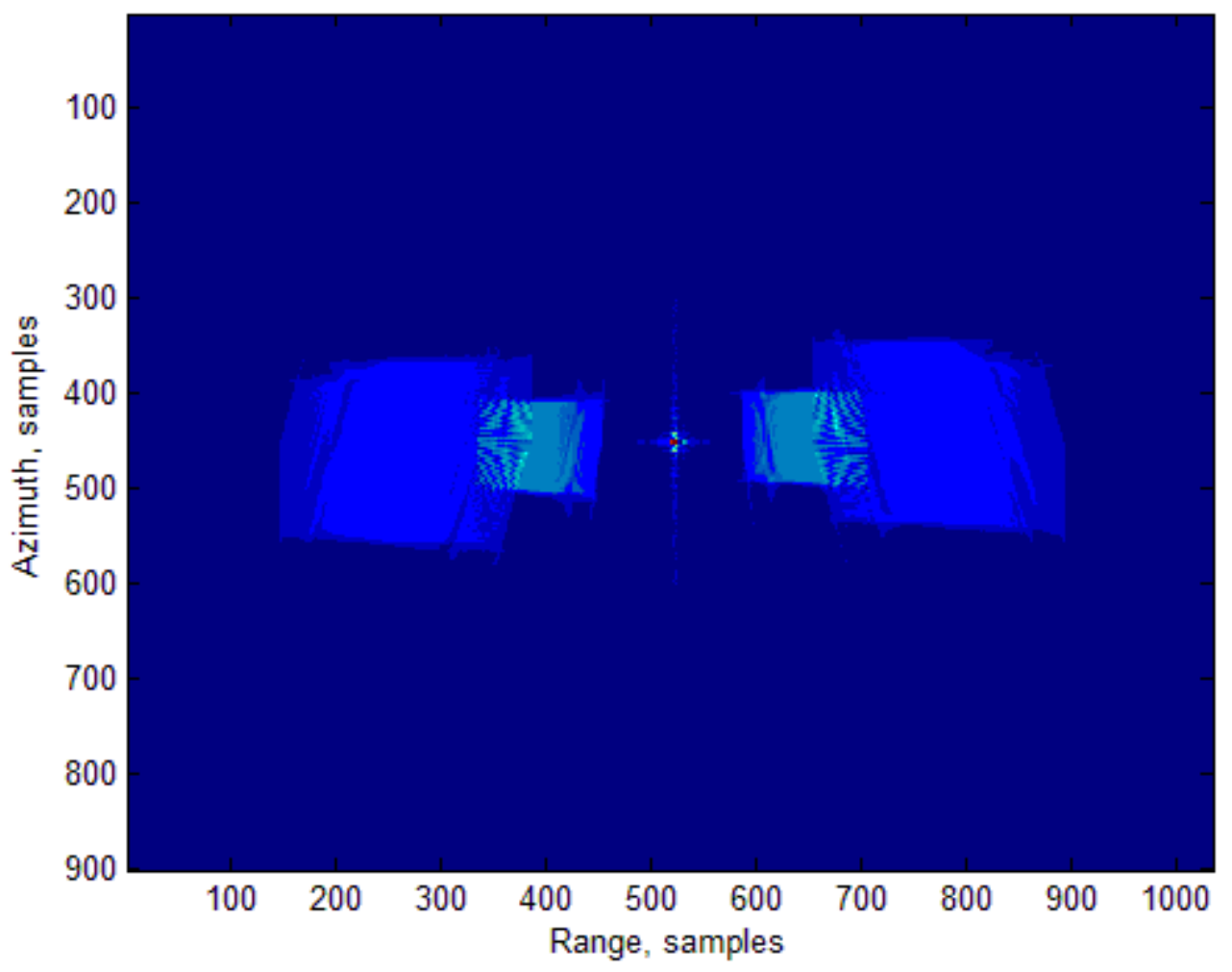

Figure 10: Final Target Signal at Varying Range Velocities

The final image convolves the signals with the azimuth reference signal, and causes the $\mathrm{X}$ and $\mathrm{Y}$ axes to be in units of samples. As expected, a target that is not moving in the range direction appears as the single point. The target location is successfully reconstructed in the target space by implementing the SAR procedure. The targets moving in the range direction appear as large rectangles, showing returns over a large area. This is consistent with the results at the previous stages of the SAR process; the targets appear at multiple range bins. Subsequently, the signals convolved with the azimuth reference signal are not an exact match at a particular azimuth bin, but instead weak signals are produced throughout multiple bins. 


\subsection{Range Velocity Detection Strategy}

Compared to the azimuth velocity, the range velocity is relatively straight-forward to detect by analyzing the returned signal. Differences become readily apparent in the first stage of the SAR process: the range compressed signal. The inverse of the slope of the traces are proportional to the velocity of the object. Therefore, the velocity of the targets can be quickly determined by calculating the inverse slopes of the traces.

The inverse slope is calculated by first determining five points on the traces; the range and azimuth pairs are found by choosing the range bin with the largest intensity value at the $250,350,450,550$, and 650 azimuth bins. By calculating the change in range per change in azimuth between the five points, the average inverse slope throughout the line can be determined. The inverse slope represents the rate that the target is moving in the range direction as platform moves across the target space.

The equation to calculate the range velocity from the inverse slope is shown as Equation 12.

$$
\text { Range velocity }=\text { inverse slope } * d x /(v p / P R F) * 100
$$

\section{Equation 12}

Since the inverse slope is given as a ratio of range bins to azimuth bins, it must be converted to the appropriate units to properly represent the range velocity. To do this, the range bin resolution needs to be stated in distance (meters), and the azimuth bin resolution stated in time (seconds). The $d x$ variable is the range resolution, and is defined as the speed of light divided by twice the baseband bandwidth $(c /(2 * B o)$. In this simulation, the baseband bandwidth is $100 \mathrm{MHz}$, so $d x$ is $1.5 \mathrm{~m}$. The azimuth resolution is 
the product of the platform velocity and the Pulse Repetition Frequency. The values used in the simulation are $200 \mathrm{~m} / \mathrm{s}$ and $300 \mathrm{~Hz}$, respectively.

\subsection{Range Velocity Detection Results}

To determine the accuracy of the equation, a point target was placed in the middle of the target space. The target was moved at range velocities from $-50 \mathrm{~m} / \mathrm{s}$ to $+50 \mathrm{~m} / \mathrm{s}$, at $10 \mathrm{~m} / \mathrm{s}$ steps. The platform was moved through the target space each time, and the Range Compressed signal was recorded and analyzed. The results of the experiment are shown in Figure 11.

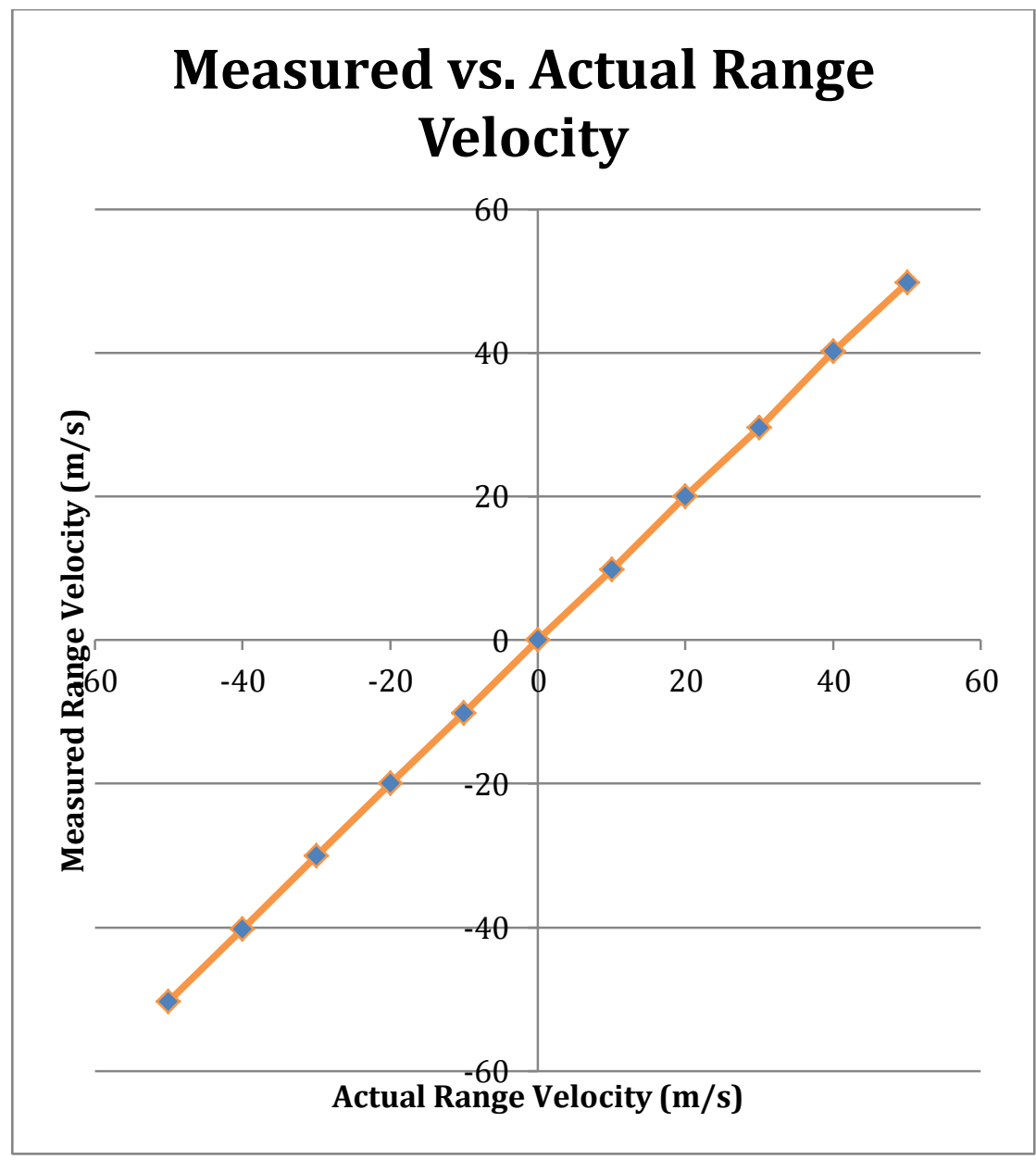

Figure 11: Measured vs. Actual Range Velocity of Target 
The percent error of each calculated velocity was also plotted against the actual range velocity of the target, and is shown as Figure 12.

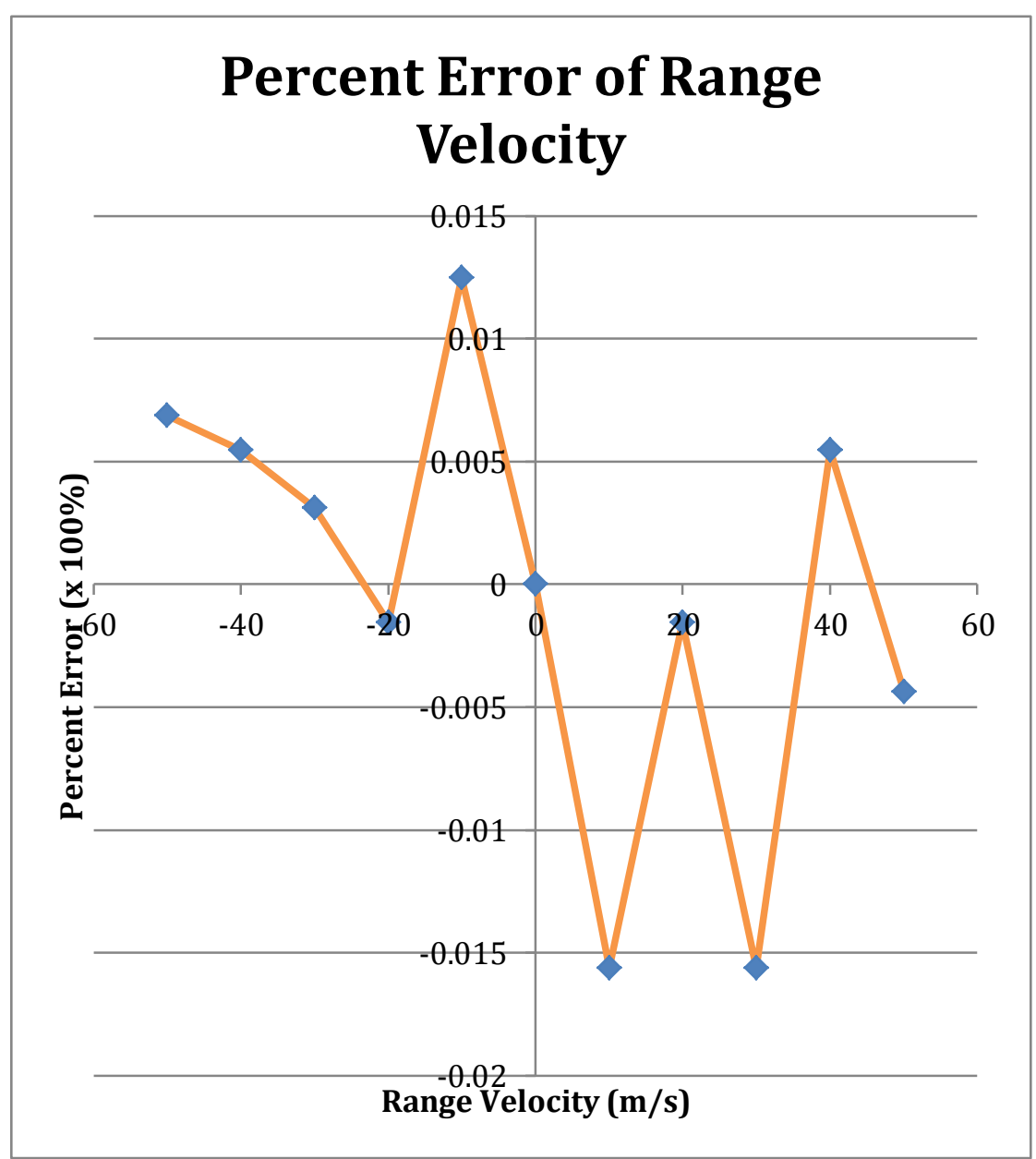

Figure 12: Percent Error of Range Velocity Calculations

The percent error of the algorithm as tested between $-50 \mathrm{~m} / \mathrm{s}$ to $50 \mathrm{~m} / \mathrm{s}$ does not exceed $2.0 \%$. The possible sources of error above could be due to the fact that the Range Compressed signal return of a target does not occur solely within a single signal range bin; some of the energy appears in bins around the actual target location. Since the algorithm determines the streak's location at each azimuth distance by looking for the 
largest value in the range bins, this spread could cause the algorithm to erroneously choose an adjacent point that also has a significant magnitude at that location. By choosing an incorrect point, the slope used in determining the range velocity will be different from the streak itself. This issue could be exacerbated in the presence of heavy noise, since this will increase the possibility that an adjacent range bin could have a larger signal magnitude than the expected bin. 


\section{AZIMUTH VELOCITY DETECTION}

An experiment was also performed to determine the behavior of targets moving in the azimuth direction. Five point targets are placed in the target space separated by 50 range bins, to prevent the signal returns from interfering with each other. The targets are configured to move in the azimuth direction at $-60,-30,0,+30$, and $+60 \mathrm{~m} / \mathrm{s}$, respectively. The signals at each step of the SAR process are analyzed to understand if varying azimuth velocities will manifest themselves in the target signals.

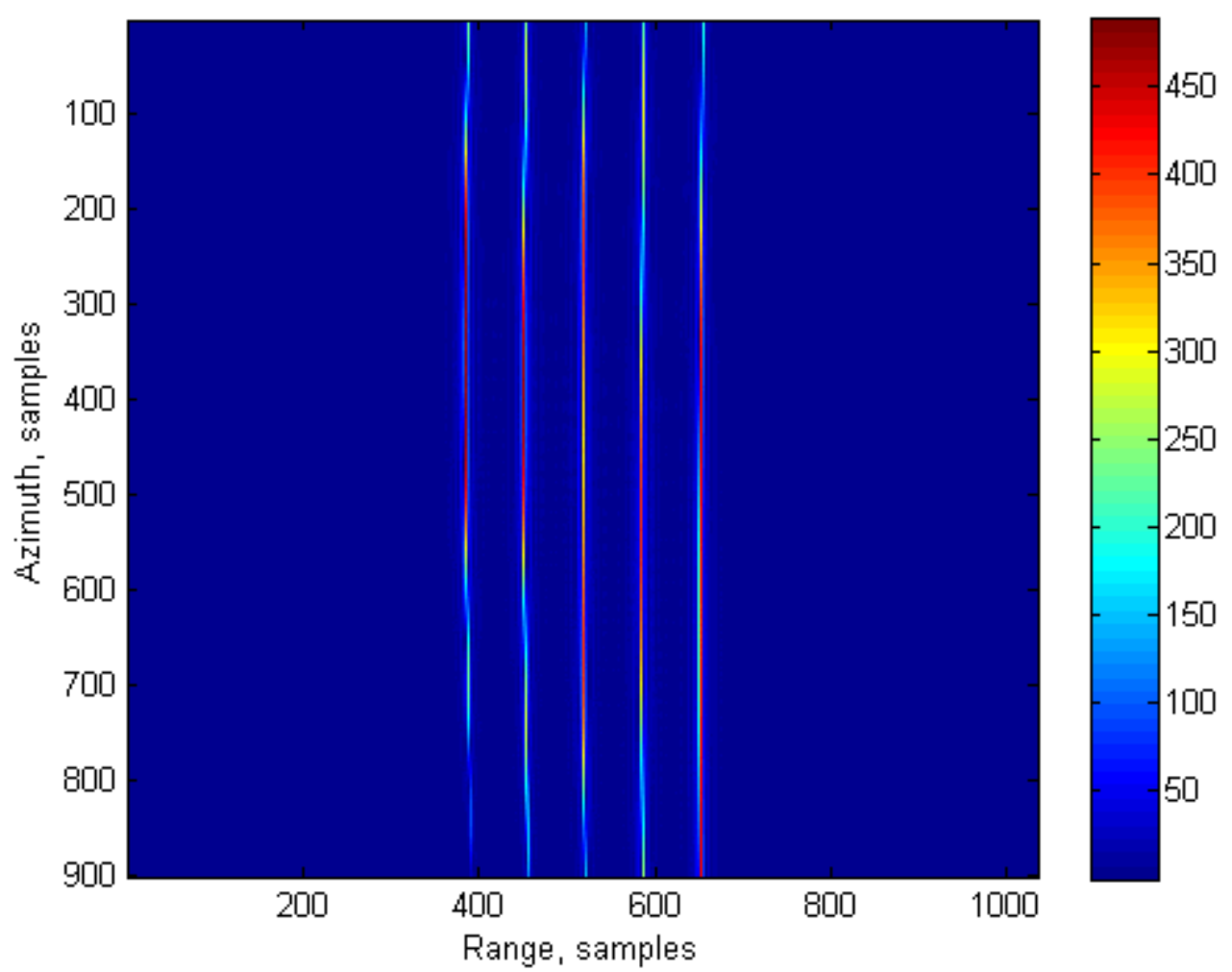

Figure 13: Range Compressed Signal at Varying Azimuth Velocities

Figure 13 is the Range Compressed signal. The targets that are moving at a negative azimuth velocity, e.g. towards the platform, have returns that taper off as the azimuth samples increase. This is due to the targets moving out of the target space sooner, and 
therefore being illuminated by the radar for a shorter period of time. Other than that, there is no obvious distinction between each point target. Since there is no change in the range distance, the returns always accumulate in the same range bin regardless of azimuth velocity.

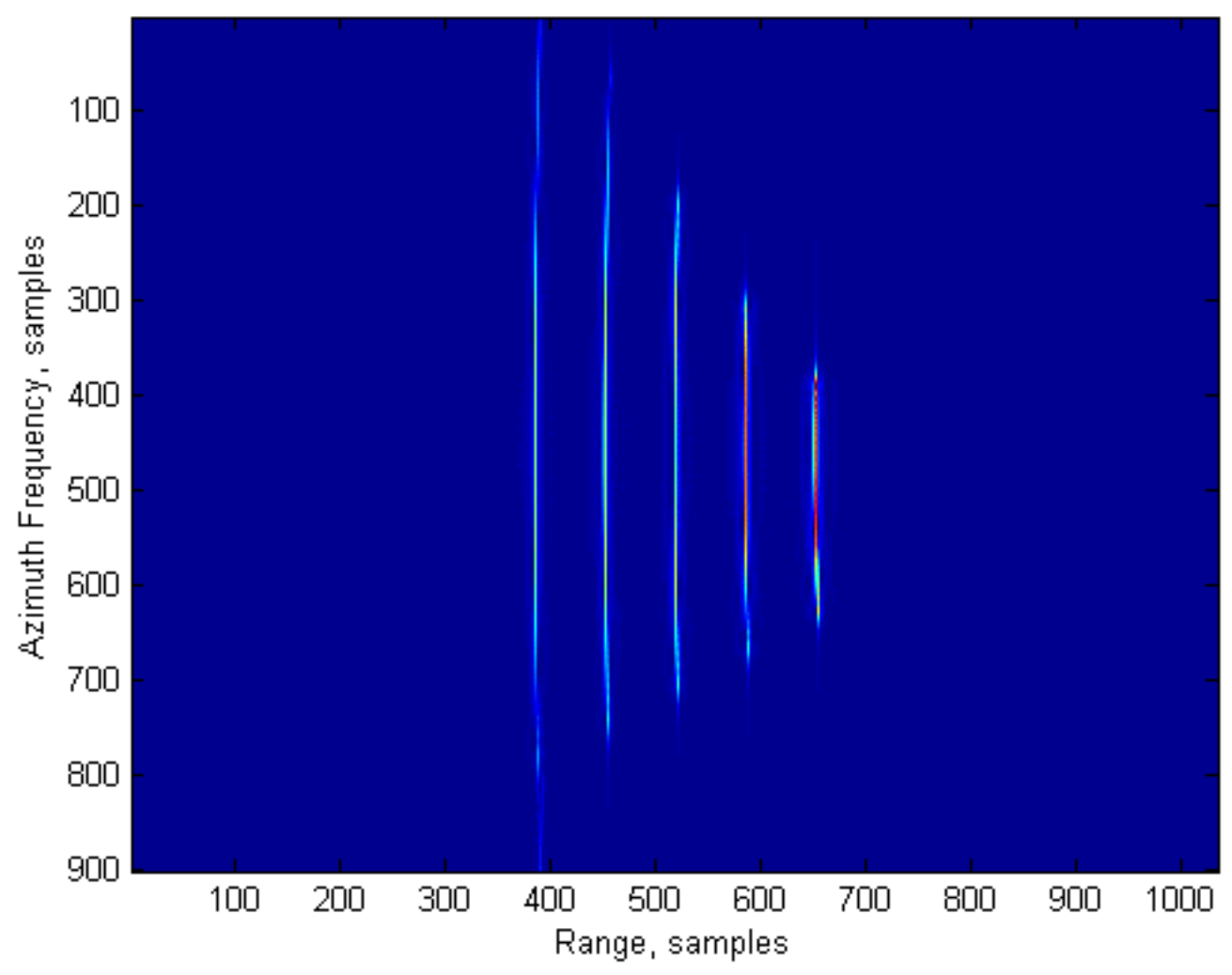

Figure 14: Azimuth Fourier Transform Signal at Varying Azimuth Velocities

The Azimuth Fourier Transform figure, or the Range-Doppler signal, is shown as Figure 14. The azimuth axis is now represented in frequency, instead of samples. In this figure, the differences between the targets are much more apparent. Compared to the stationary target, the targets with negative azimuth velocities have much longer tracks, while the targets with positive azimuth velocities have shorter tracks. Targets that are moving in the opposite direction of the platform will experience larger Doppler shifts, since there is a larger difference in velocity between the two. Conversely, targets moving in the same 
direction of the platform will see smaller Doppler shifts. This causes a larger and smaller range of azimuth frequencies to be received by the platform, respectively.

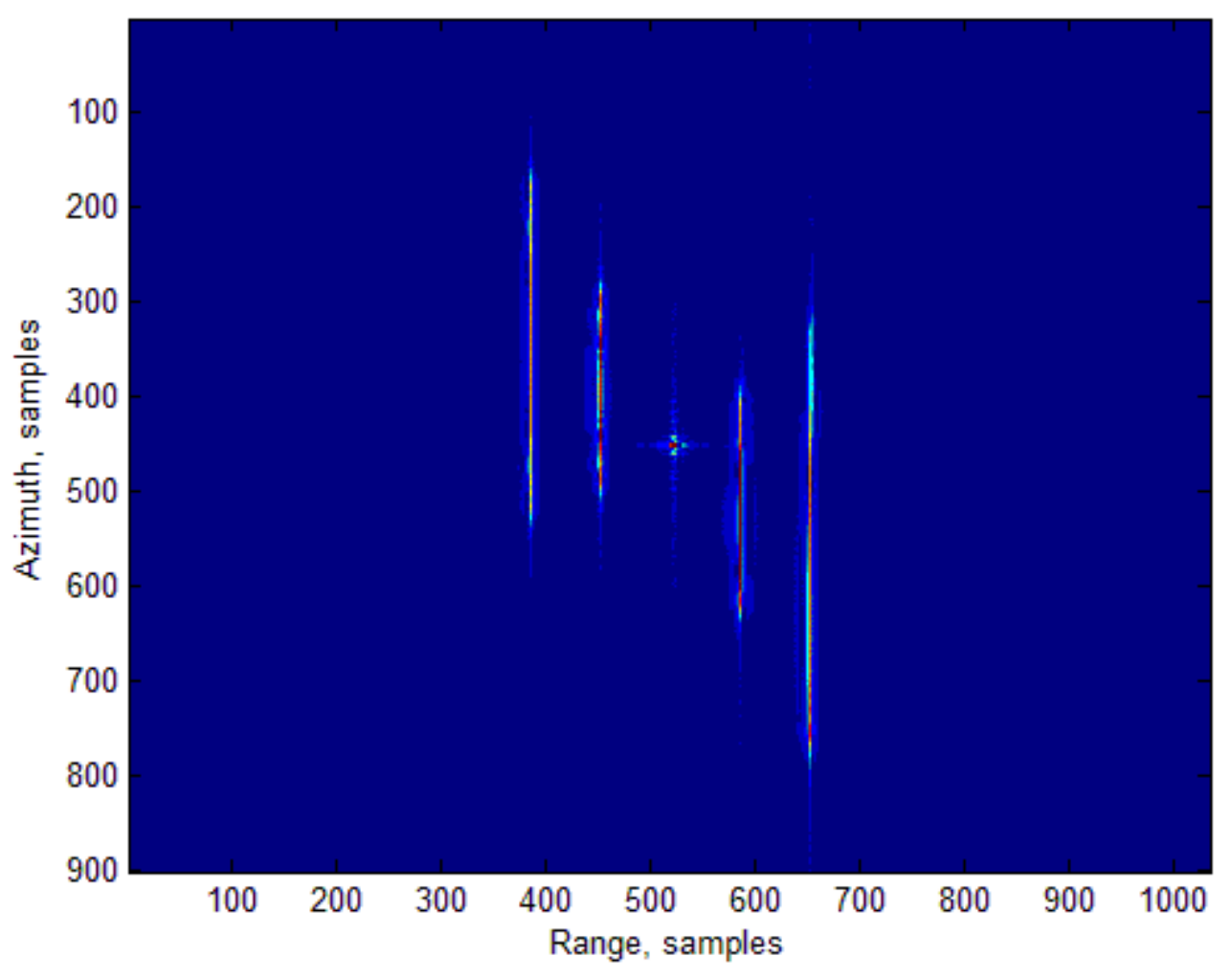

Figure 15: Final Target Imagining Signal at Varying Azimuth Velocities

The final target image is shown as Figure 15. As expected, the target not moving in the azimuth direction appears as a dot and is fully represented by a single azimuth and range bin. Targets that had a nonzero azimuth velocity appear as a line spanning multiple azimuth bins, with the length roughly proportional to the target's azimuth velocity. Each target's trace shows up in a single range bin, since the targets maintain the same range distance while the platform moves through the target space. 


\subsection{Azimuth Velocity Detection in the Range-Compressed Space}

As shown in Section 1.2.2, the received beam pattern from the target normally

approximates a sinc ${ }^{2}$ function. The signal is strongest when the platform is directly orthogonal to the target, but the side lobes of the signal results in some energy being received when the target is a certain azimuth distances away. Since the side lobe locations are fixed, they can be used to determine the azimuth velocity of the target.

The strongest return occurs when the slant range is zero, and the platform is directly over the target. As the platform moves through the target space, the azimuth distance between it and the target increases. Certain slant ranges cause local maxima of the received signal, since that is where the side lobes are located. The platform moves parallel to the azimuth plane, so the local maxima appear at fixed positions in the azimuth direction. When the target moves at a positive azimuth velocity, these local maxima show up closer together since the absolute velocity between the target and the platform is increased. Conversely, when the target moves at a negative azimuth velocity, the local maxima show up farther away from one another, since the absolute velocity has decreased.

Targets moving in the azimuth direction appear on the range compressed signal stage as vertical streaks, as shown in Figure 16. 


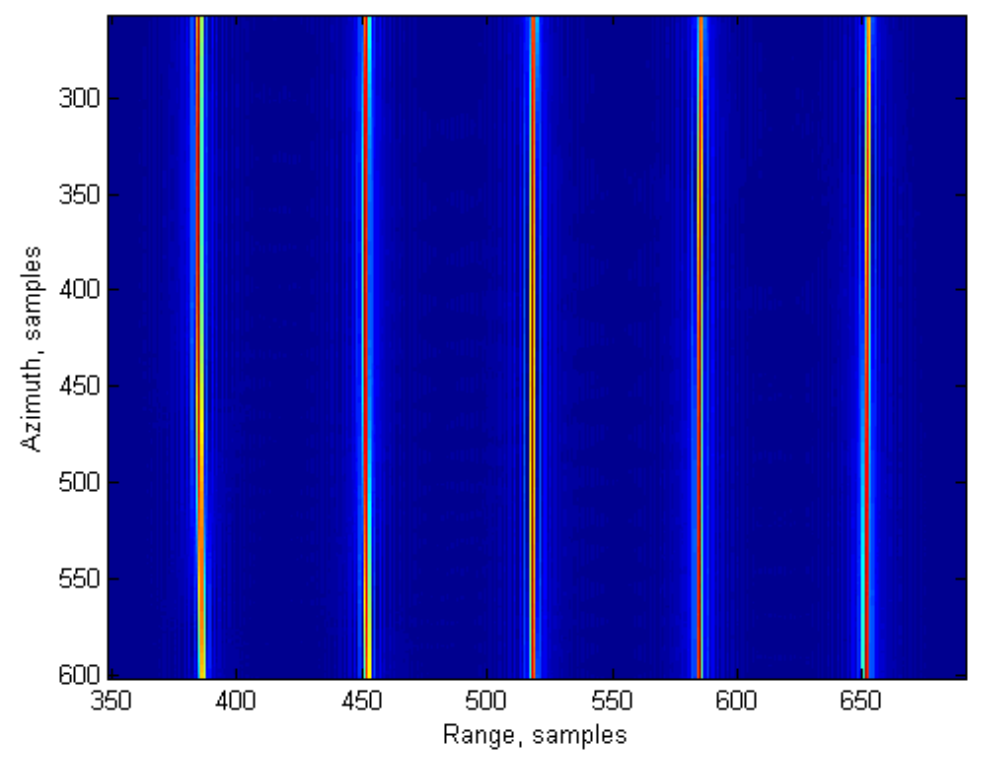

Figure 16: Expanded View of Range Compressed Signal

At first glance, there is no discernable difference between the targets moving at different velocities. However, when the magnitude of the trace is plotted against the azimuth sample, the effect of azimuth velocity becomes much more apparent.

The baseline example is when the target does not have an azimuth velocity. The range compressed signal magnitude versus the azimuth bin is shown in Figure 17. 


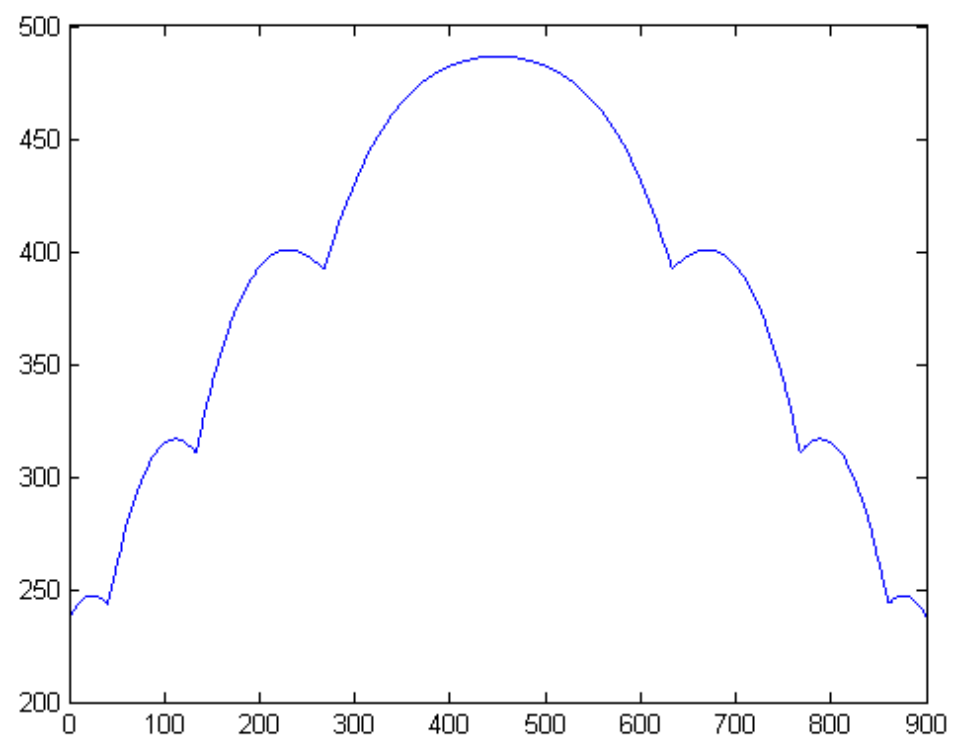

\section{Figure 17: Range Compressed Signal vs. Azimuth Bin}

The waveform is symmetric about the middle of the graph, at the $450^{\text {th }}$ azimuth bin. The distance between each side lobe to the main lobe is about 230 azimuth bins. In comparison, the waveform for a target with a positive azimuth velocity is more stretched out and the maximum is also shifted to the right. The waveform of a target moving at +30 $\mathrm{m} / \mathrm{s}$ in the azimuth direction is shown in Figure 18. 


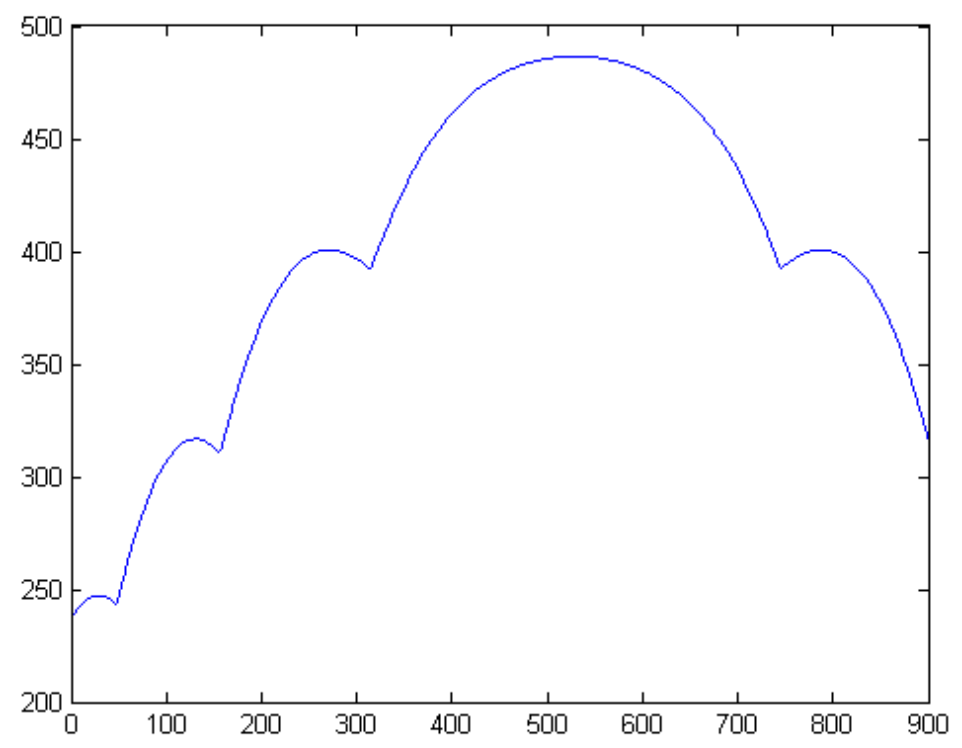

\section{Figure 18: Range Compressed Signal vs. Azimuth Bin, With Positive Velocity}

In this waveform, the maximum occurs at around 530, which is larger when compared to 450 when the target is not moving. This behavior is consistent with our expected results; since the target is moving in the same direction as the target platform, a larger number of azimuth bins need to be traversed before the platform catches up to the target and the SAR is directly orthogonal to the target. In addition, the distance between the main lobe and the side lobes is about 250 , which is larger than the stationary case. This is due to the reduced relative velocity between the SAR platform and the target. The lower relative velocity causes the target to appear at the side lobe locations later. 


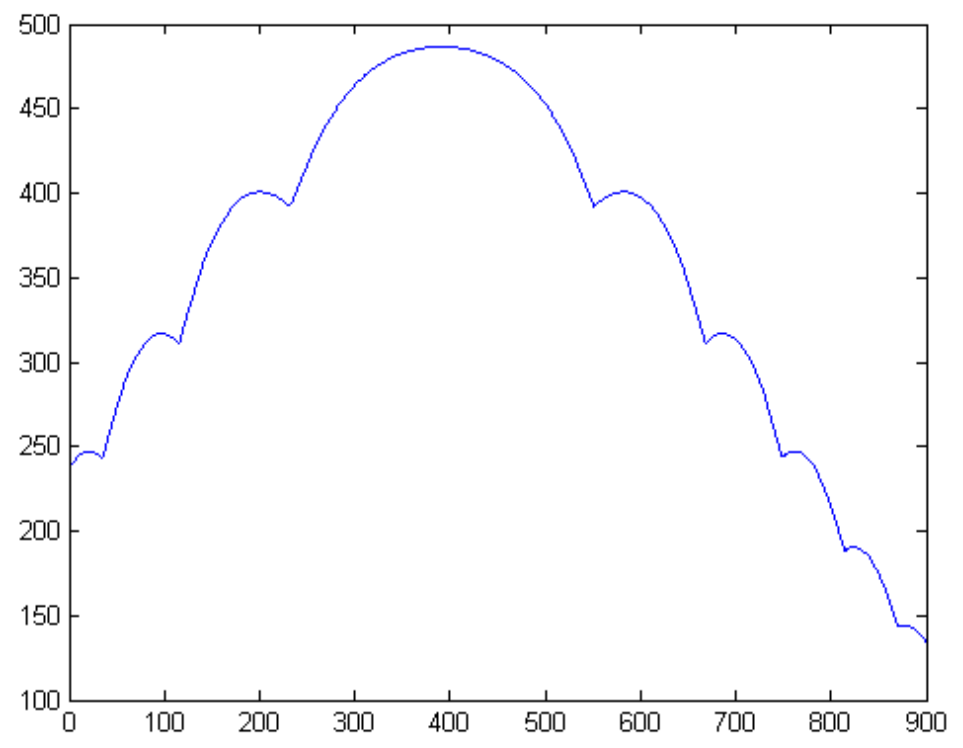

\section{Figure 19: Range Compressed Signal vs. Azimuth Bin, With Negative Velocity}

Conversely, Figure 19 is of the same target moving in the negative azimuth velocity. Due to the increased relative velocity between the target and the SAR platform, the target reaches a particular distance away from the platform earlier than if it was stationary. The main lobe of the graph is positioned at 390. Likewise, the two side lobes are positioned at 200 and 580. As a result, the distance between the side lobe and main lobe is 190.

Based on the above findings, the side lobe to main lobe distance is positively correlated to the target's azimuth velocity. The absolute position of the sensor is also affected by the target's velocity, but it is also dependent on other factors, like the starting position of the target. Therefore, the usage of the lobe distance to determine the target's azimuth velocity was explored.

In this implementation, the distance between the left side-lobe and the main lobe was considered as the lobe distance. A point target was placed in the center of the target space. The target was then moved at varying azimuth velocities, and the resulting 
waveforms analyzed and the lobe distances recorded. The azimuth velocities were chosen to be $-50 \mathrm{~m} / \mathrm{s}$ to $+50 \mathrm{~m} / \mathrm{s}$, at $5 \mathrm{~m} / \mathrm{s}$ intervals. The graph of the recorded lobe distances plotted against the azimuth velocity is shown in Figure 20.

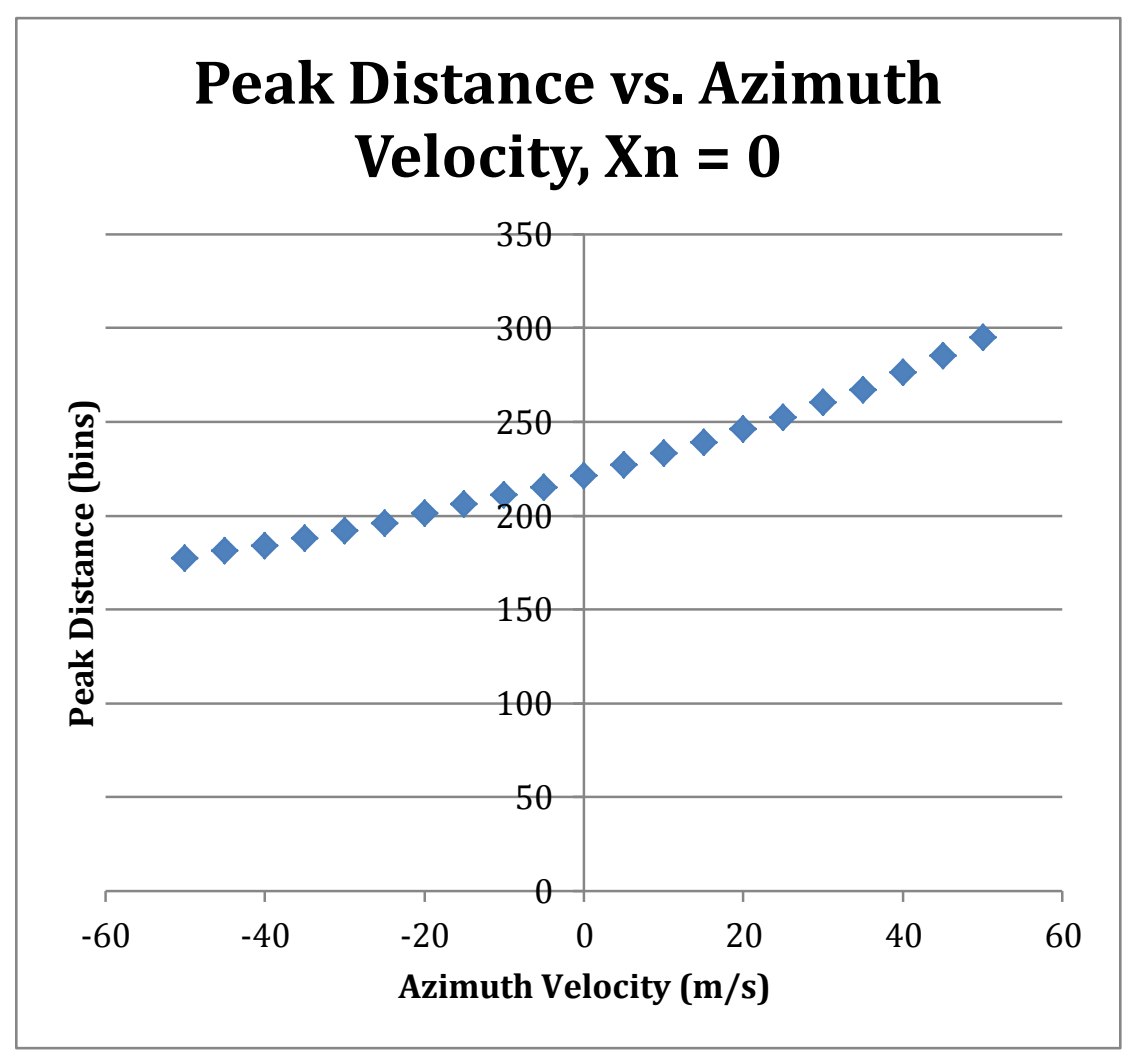

Figure 20: Measured Peak Distance vs. Target Azimuth Velocity

Alternatively, the target's azimuth velocity versus the resulting lobe distance is shown in Figure 21. 


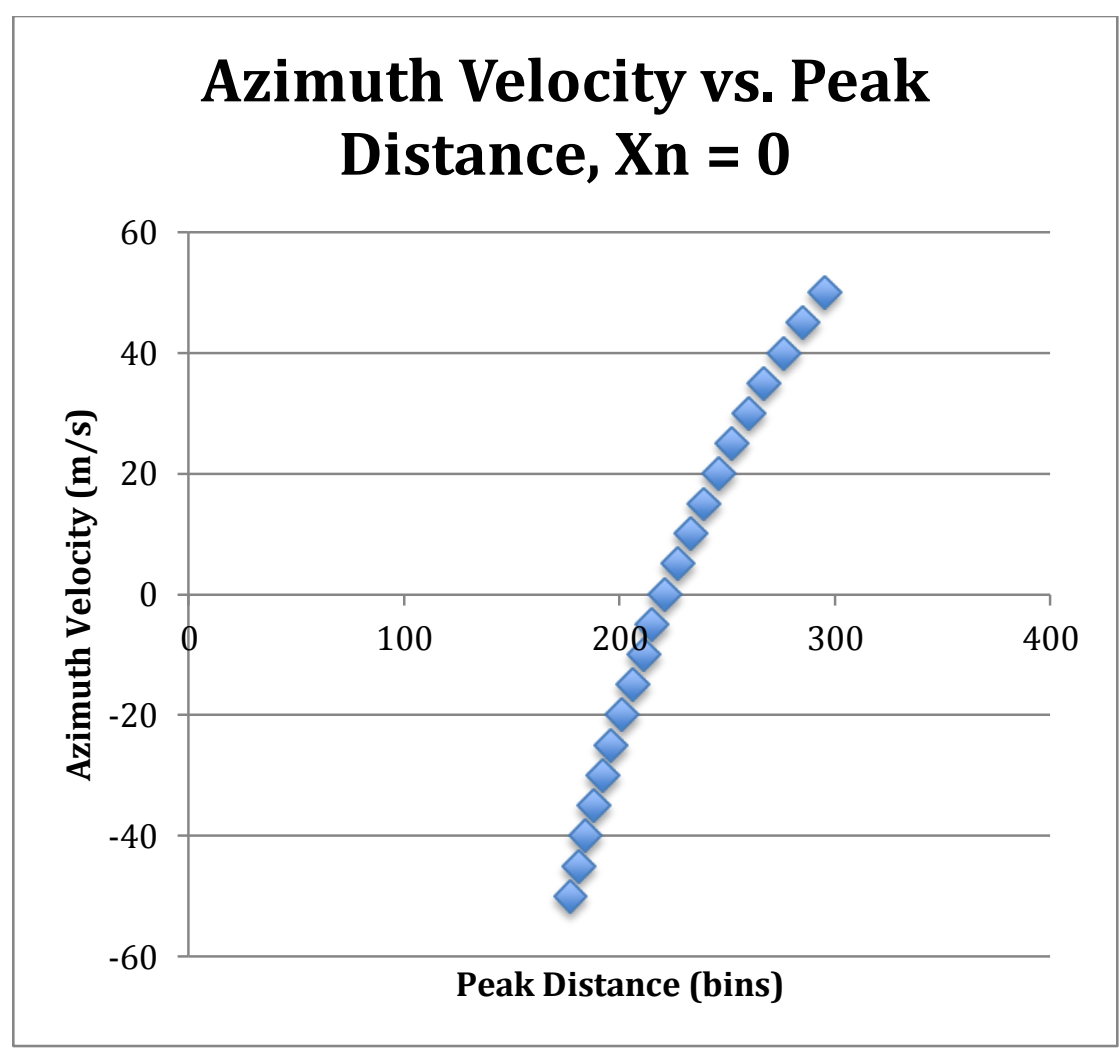

Figure 21: Target Azimuth Velocity vs. Measured Peak Distance

The relationship between the lobe peak distance and the target's azimuth velocity can be approximated by a second-order equation. The polyfit function in MATLAB was used to calculate the best-fit equation, which is shown as Equation 13.

$$
\begin{gathered}
v=-0.0036581 d^{2}+2.55643347 d-386.6495116 \\
\text { Equation } 13
\end{gathered}
$$

$v=$ estimated azimuth velocity of the target

$d=$ lobe peak distance 


\subsection{Azimuth Velocity Detection in the Range-Doppler Space}

In addition to the Range-Compressed space, a target's azimuth velocity causes noticeable effects in the Range-Doppler space. Although generating the Range-Doppler image will require additional processing time and is more computationally intensive, analysis on the Range-Doppler may still be an effective strategy to determine the target's azimuth velocity.

Due to the nature of the SAR process, the signal received by the platform will experience a Doppler shift. The equation for the apparent frequency of the incoming wave, as seen by the platform, is shown below as Equation 14 .

$$
f=\left(\frac{c+v_{r}}{c+v_{s}}\right) f_{0}
$$

\section{Equation 14: Doppler Shift Experienced By a Moving Receiver}

$v r=$ receiver velocity

$v s=$ source velocity

Since the SAR platform is moving sideways alongside the target at a constant azimuth velocity, the component of the velocity that is in the direction of the target varies according to the position of the platform. When the platform first enters the target space, it is far away from a target positioned in the middle of the space. This causes the platform to be moving roughly as fast towards the target as it is in the azimuth direction. However, as the platform approaches the target, the angle between the platform to the target and the azimuth direction increases. The relative velocity between the platform and the target decreases, since the platform is no longer headed directly towards it. There is a 
corresponding decrease in the signal frequency received by the SAR platform.

Eventually, the vector from the target to the platform becomes orthogonal to the azimuth direction, and the apparent velocity between the target and the platform becomes zero. The Doppler shift also becomes zero as a result.

As the platform moves past the target, the angle between the target and the azimuth direction begins to decrease. When this happens the relative velocity between the target and the platform becomes more and more negative, resulting in a decreased signal frequency at the SAR platform. The frequency continues to decrease in this fashion until the SAR platform leaves the target space.

As a consequence, the SAR platform will receive a range of frequencies as the platform moves through the target space. The Range-Doppler plot is generated by applying the Fast Fourier Transform, and displays the frequency spectrum of the azimuth signal in the vertical axis. Therefore, the streak that appears for each target represents the range of signal frequencies associated with that target as the platform moves through the target space.

Varying target azimuth velocities therefore affect the range of frequencies seen by the platform. When the target has a positive azimuth velocity, there is a small range of frequencies that the target experiences as it moves through the target space. As the target is moving in the same direction as the platform, so the relative velocity between them is reduced by the target's azimuth velocity. Conversely, the range of frequencies seen by the platform increases if the target has a negative azimuth velocity, since the relative velocity between the platform and the target is increased. Figure 22 is the Range-Doppler 
waveform of 5 targets moving with various azimuth velocities. From left to right, the targets are moving at $-60,-30,0,+30$, and $+60 \mathrm{~m} / \mathrm{s}$ in the azimuth direction, respectively.

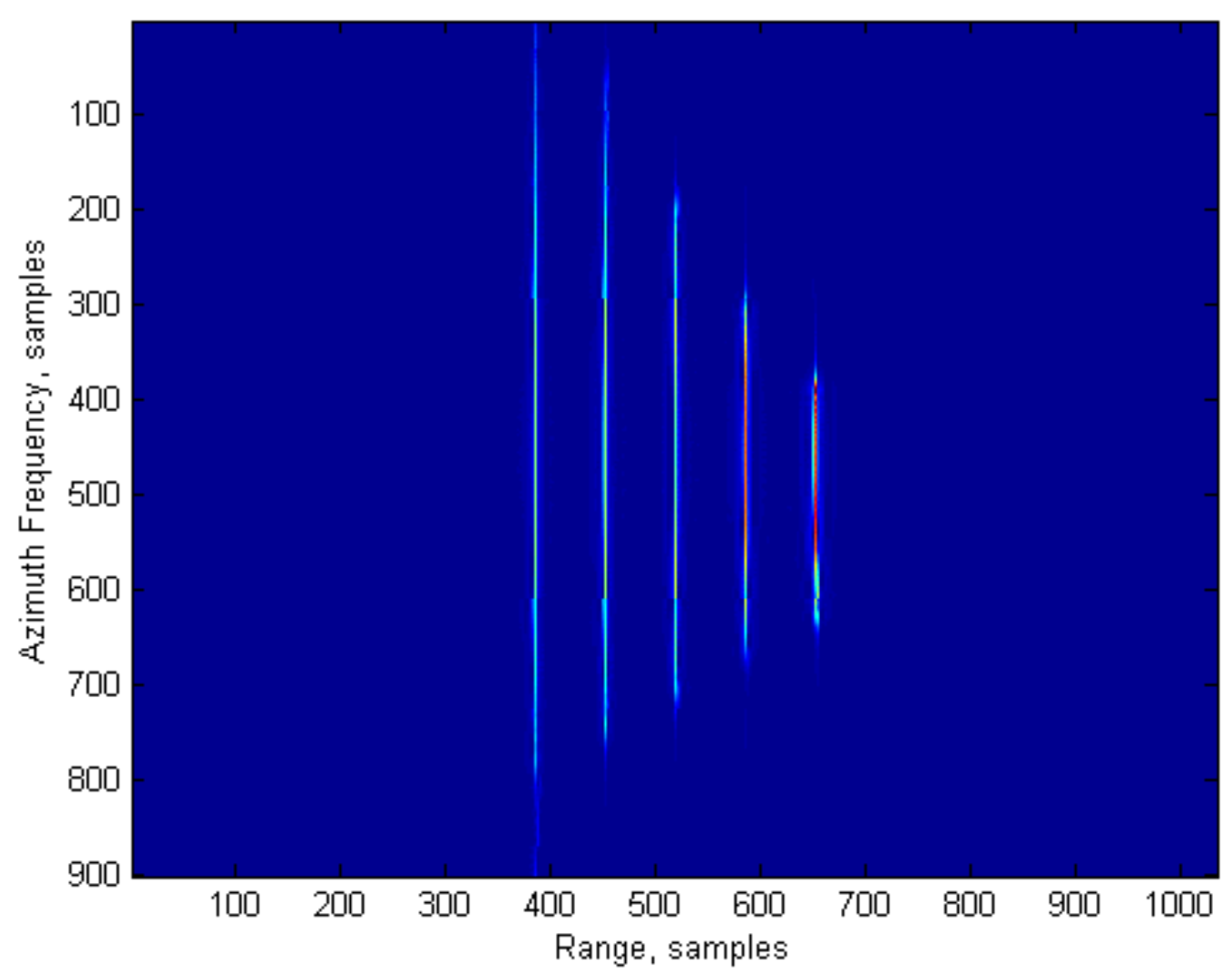

Figure 22: Range-Doppler Plot of Targets With Azimuth Velocities

The range of frequencies shown on the Range-Doppler plot for each target is as expected. Compared to the stationary target (center streak), the target with a negative azimuth velocity has a longer streak, while the one with a positive velocity has a shorter streak.

From the results, there are a couple characteristics that may be used to differentiate each streak from each other. In this paper, two characteristics were analyzed to attempt to reliably correlate the target's azimuth velocity with the resulting Range-Doppler signature: the streak's peak value and its variance. 
As the azimuth velocity of the target changes, the peak value of the Range-Doppler signal increases too. The same Range-Doppler data shown previously is shown as Figure 23, this time with tighter axes around the origin.

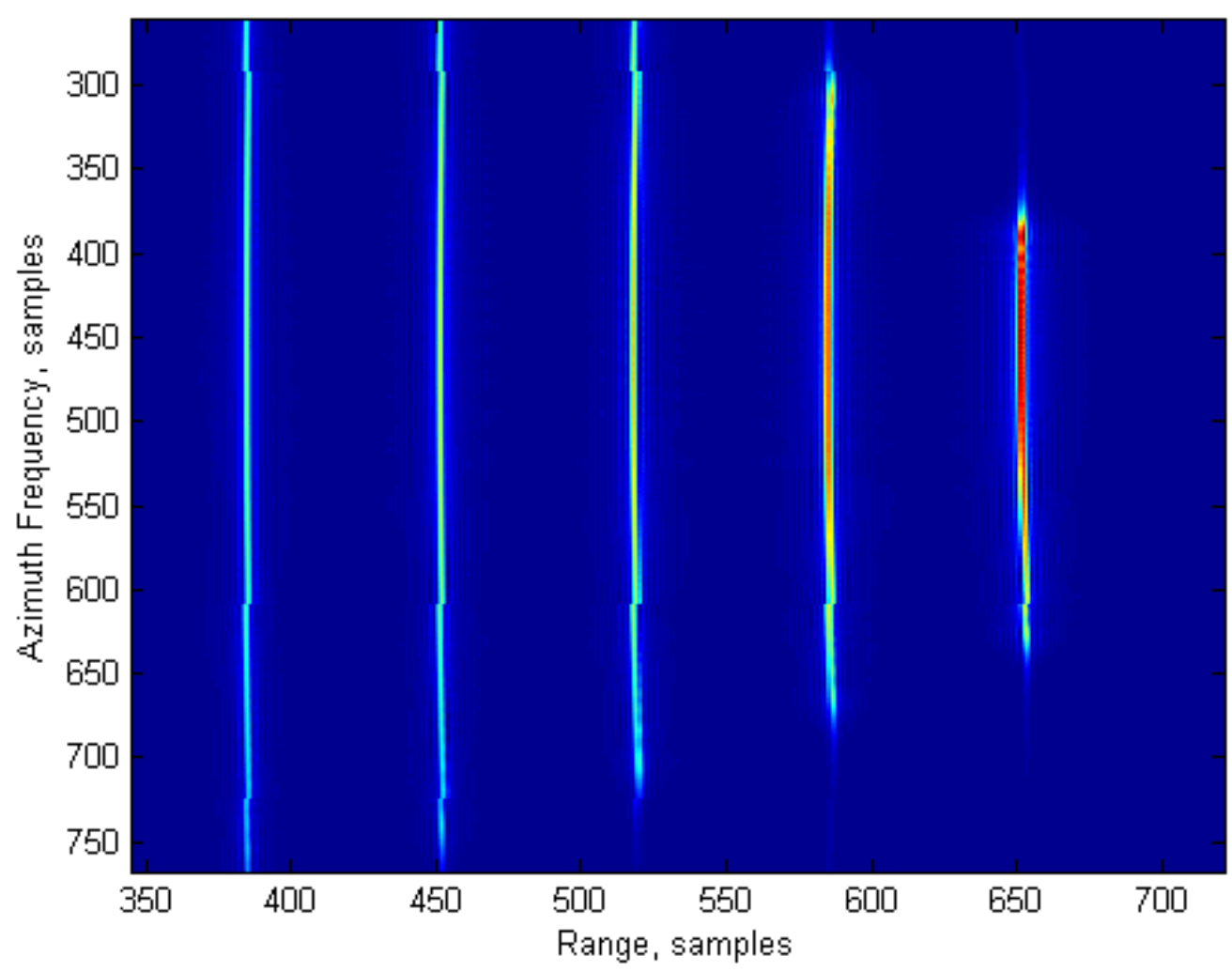

Figure 23: Magnified Range-Doppler Plot of Targets With Azimuth Velocities

The shorter streaks that appear at higher azimuth velocities, such as the two streaks on the right, exhibit higher peak values. This is due to the compression of the frequency range of the received signals, causing the peak value to increase. The opposite behavior is shown by the two streaks on the left, which are generated by targets with negative azimuth velocities. In those cases, the energy of the received signals is spread across a larger 
bandwidth, reducing the peak intensity. Analysis of the peak value can therefore be used to determine the azimuth velocity of the target.

\subsection{Range-Compressed Azimuth Velocity Detection Results}

Analyzing the behavior of the Range Compressed signal as the azimuth distance of the platform varies can approximate the target's azimuth velocity. The waveform of the signal has a main lobe and side lobes on either side, due to the $\operatorname{sinc}^{2}()$ behavior of the signal. The distance between the main lobe and the side lobes can be correlated to the target's azimuth velocity, since movement in the azimuth direction results in a compression or elongation of the waveform.

The MATLAB implementation of determining the location of the side lobes and main lobes uses a system of flags to keep track of the status of the signal at the azimuth bin currently being evaluated. The peak of the main lobe is first found by finding the maximum value of the streak. Once the index of the maximum value is found, the algorithm will step through backwards from when the maximum value occurred to the first element of the array. At each index, if the flag has not been set, the value of that index is compared to the value of the previous index. If the current value is lower, it means that the index is not the local minimum. However, if the current value is higher than the previous value, then the local minimum has been found and the flag is set. The algorithm continues traversing the array; however, with the flag set, the algorithm begins to check if the current index is lower than the previous value instead. Once a value is found to be lower than the previous, the current index is identified to be the peak of the left side lobe. 
To characterize the effectiveness of this approach, a point target was placed in the middle of the target space and moved at azimuth velocities from $-50 \mathrm{~m} / \mathrm{s}$ to $+50 \mathrm{~m} / \mathrm{s}$, at $5 \mathrm{~m} / \mathrm{s}$ steps. At each target azimuth velocity, the platform was moved across the target space and the Range Compressed signal of the target was analyzed. The azimuth velocity of the target was then calculated using the curve-fit equation above. The comparison between the calculated values and the actual velocities are shown in Figure 24.

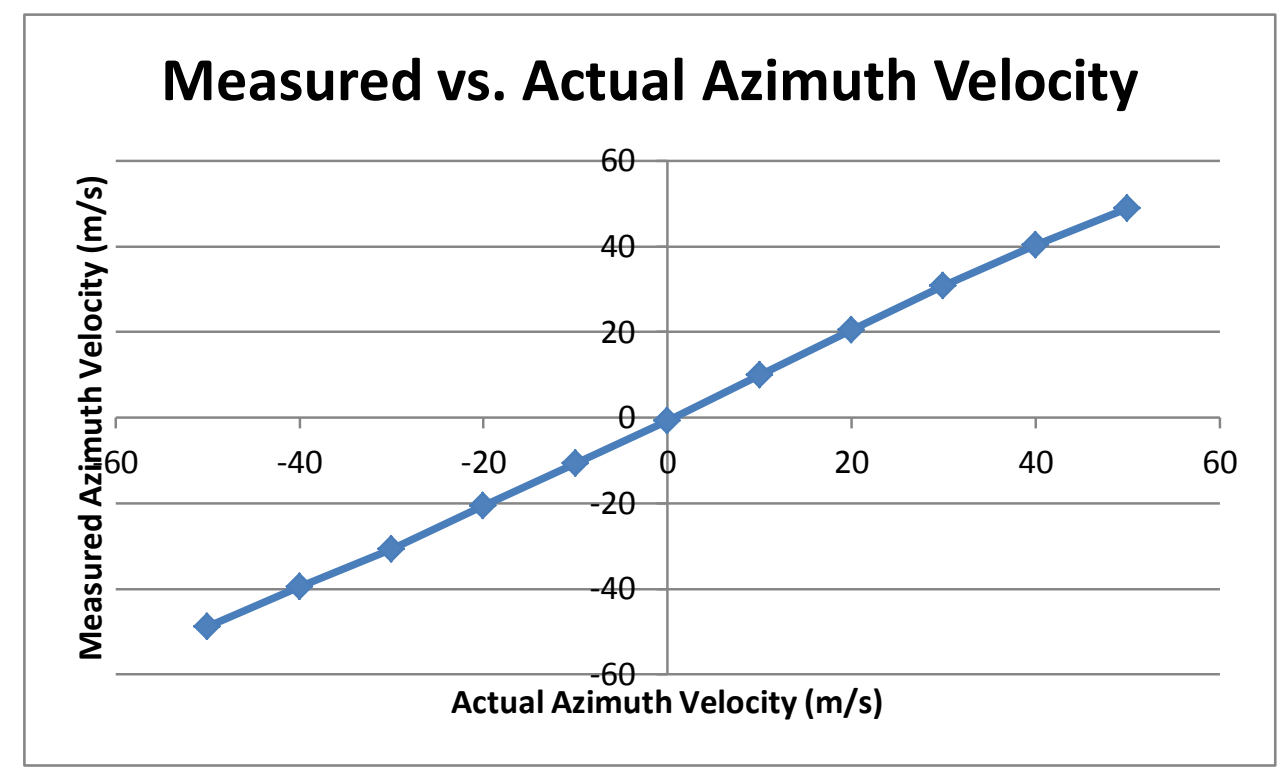

Figure 24: Measured vs. Actual Azimuth Velocities of Targets 
Figure 25 shows the absolute error in the azimuth velocity calculation.

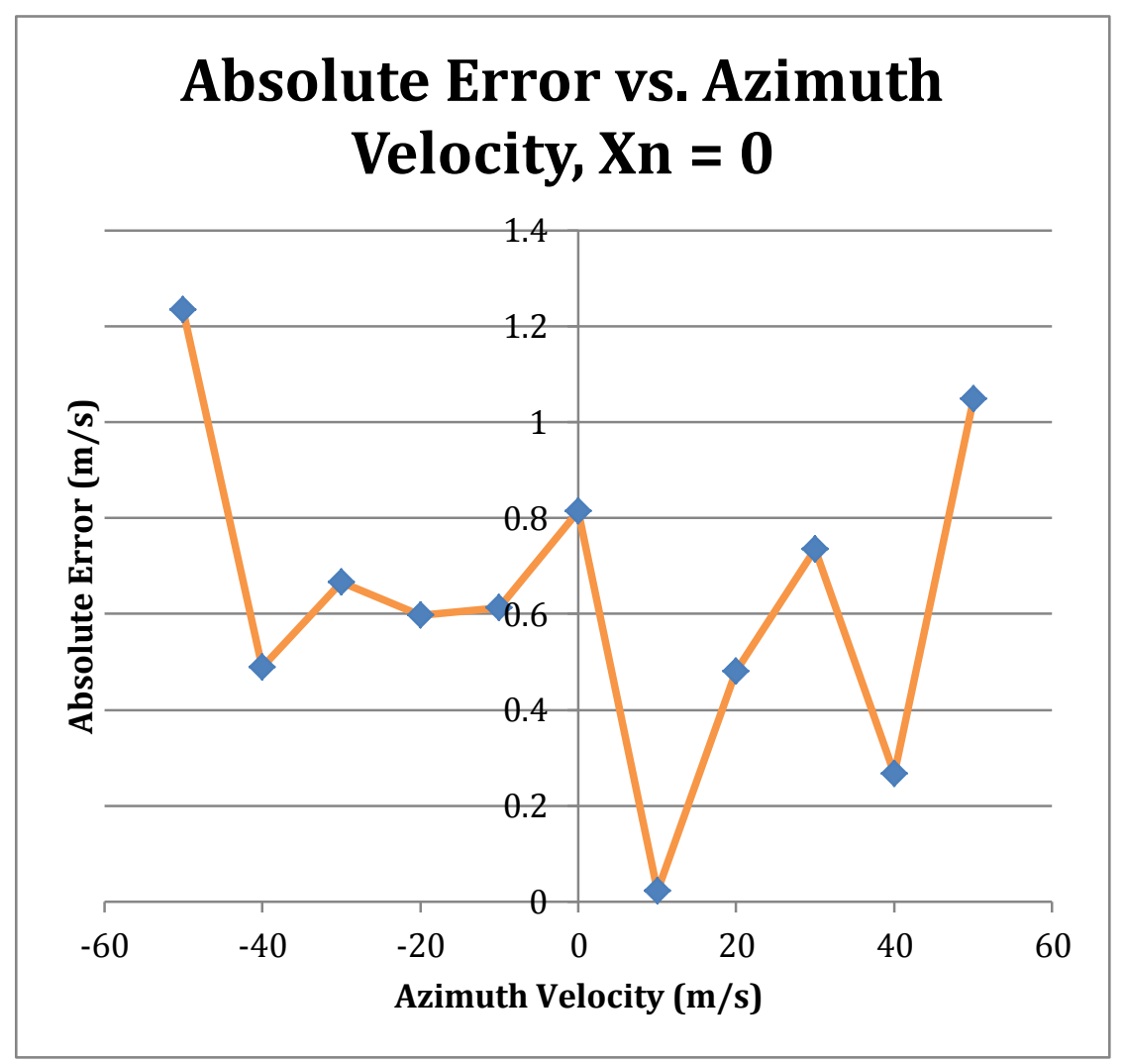

Figure 25: Absolute Error of Range-Compressed Azimuth Velocity Calculations 
The graph of the percent error at each azimuth velocity is shown as Figure 26.

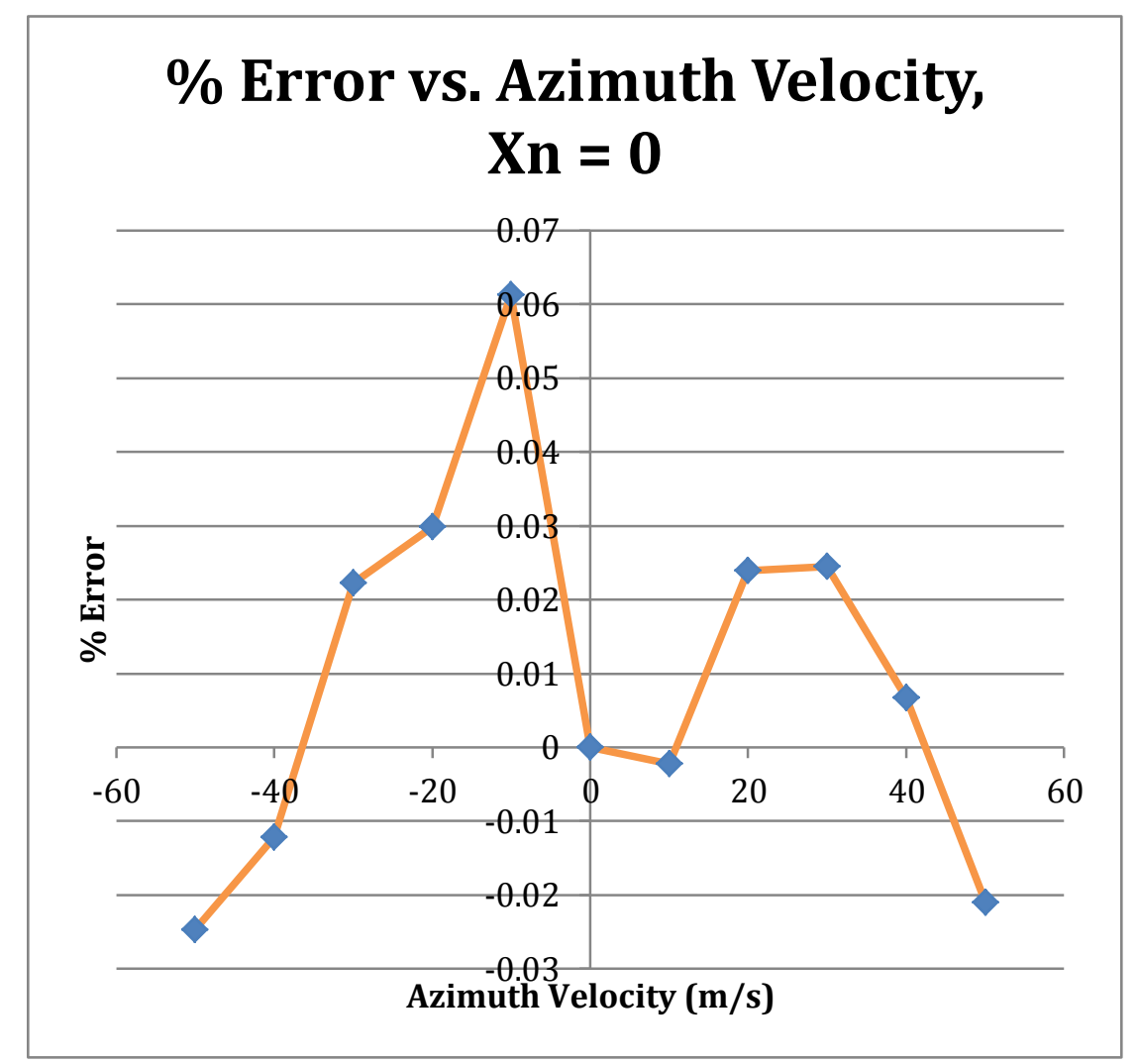

Figure 26: Percent Error of Range-Compressed Azimuth Velocity Calculations

The three graphs above shows that for the data points we have chosen above, the error does not exceed $1.5 \mathrm{~m} / \mathrm{s}$. The maximum $\%$ error in this data set is $6.125 \%$, which occurred when the target was moving at $-5 \mathrm{~m} / \mathrm{s}$ in the azimuth direction. Based on the above findings, it seems like the absolute error remains roughly constant across varying azimuth velocities. Therefore, it looks like a majority of the error is due to quantization of the signal into discrete azimuth and range bins. The main lobe to side lobe distance is always represented as an integer number of azimuth samples, contributing to the error. The peak positions themselves are represented by a specific azimuth bin, which may 
introduce error if the peaks of the main lobe or side lobe do not fall exactly on that azimuth location.

When Additive White Gaussian Noise (AWGN) is introduced into the system, the main lobe and side lobes of the Range Compressed signal waveform at the target's range bin become harder to determine. As an example, Figure 27 shows the Range Compressed Signal of a stationary target in the middle of the target space, when AWGN is not introduced in the system.

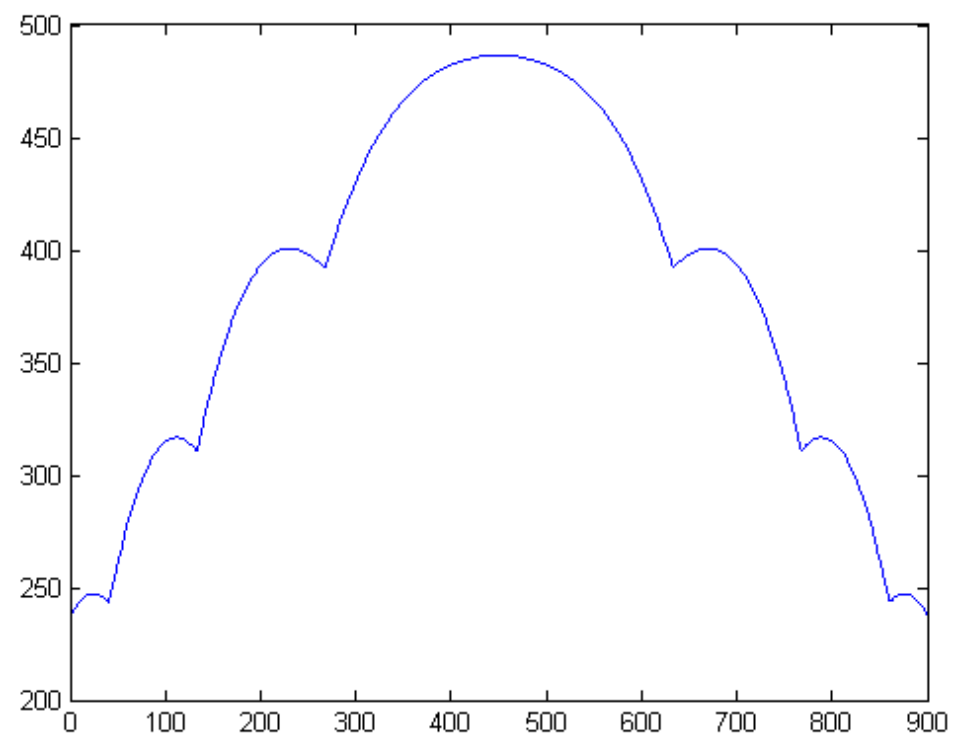

Figure 27: Range Compressed Signal vs. Azimuth Bin, no AWGN

When AWGN with a standard deviation of 0.75 is added, Figure 28 shows the resulting signal. 


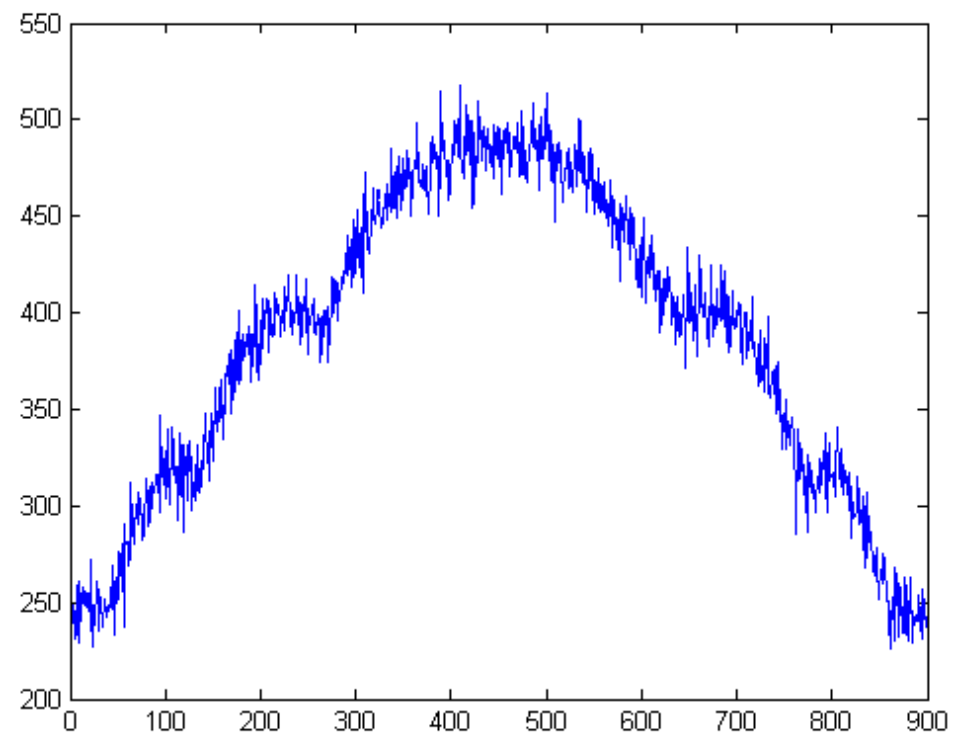

Figure 28: Range Compressed Signal vs. Azimuth Bin, AWGN added

The signal with the AWGN added will not be able to analyzed using the current MATLAB implementation of determining the location of the main and side lobes. In order for the algorithm to work, the signal will need to be processed to remove the noise in the signal and reveal the peaks of the main and side lobes.

When the point target is placed at different range locations in the target space, the shape of the Range-Compressed signal appears differently. There appears to be three variations of waveform shapes that the signal takes depending on the starting range location of the target. The following three examples are of a point target moving at an azimuth velocity of $-30 \mathrm{~m} / \mathrm{s}$. When the target is placed at $\mathrm{Xn}=58$ in the range direction, the waveform appears as Figure 29. 


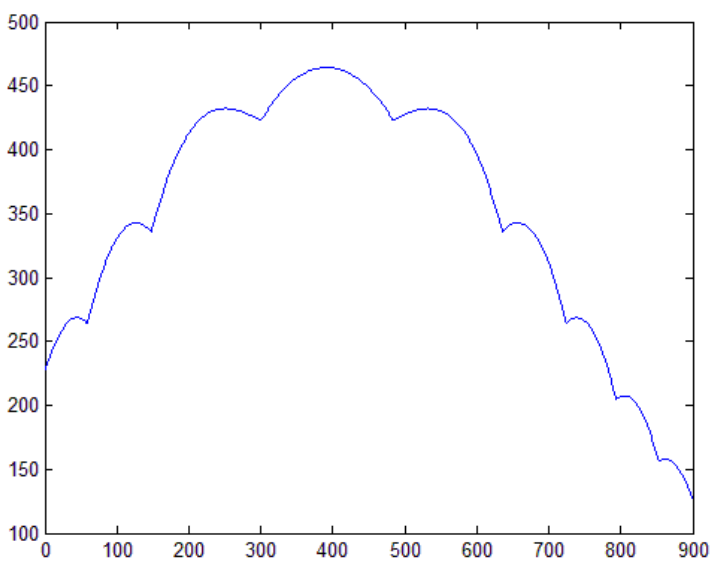

Figure 29: Range Compressed Signal vs. Azimuth Bin, $\mathrm{Xn}=58$

Compared to the waveform when the target started at the origin, the side lobes are higher, and the waveform is slightly compressed in the $y$ direction, causing the left and right side lobes to be positioned closer to the main lobe.

Figure 30 shows the Range-Compressed signal when the target is placed at $\mathrm{Xn}=59$ in the range direction.

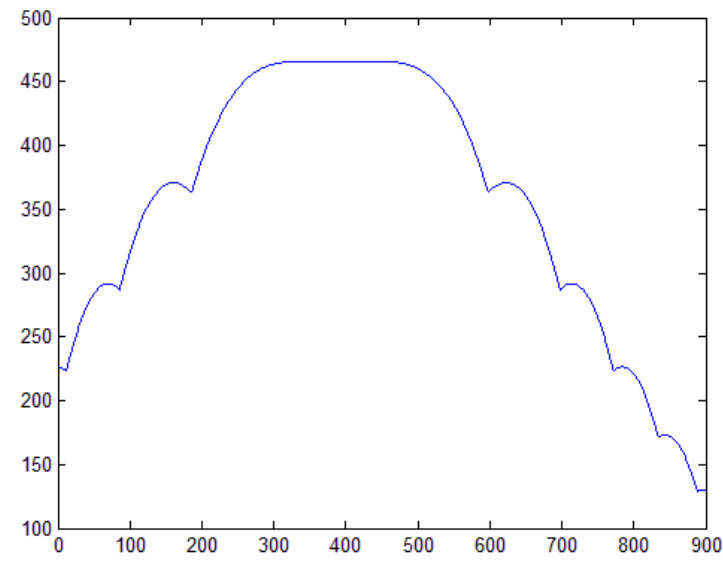

Figure 30: Range Compressed Signal vs. Azimuth Bin, $X n=59$

At this starting range direction, the waveform appears much different than the other two. The main lobe has been stretched into two lobes with a local minimum occurring between 
them. The waveform has also been stretched in the $y$ direction, causing the side lobes to occur farther from the main lobe.

For the third example, the target was placed at the $\mathrm{Xn}=60$ location. The resulting Range Compressed signal is shown as Figure 31.

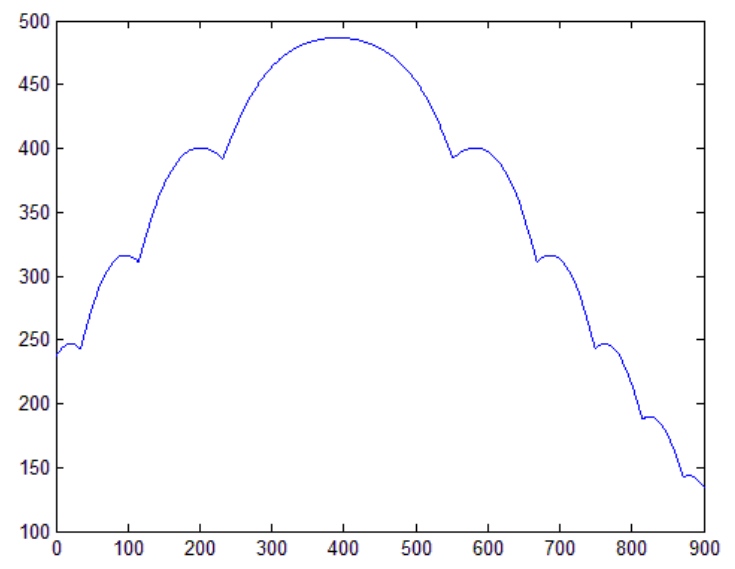

Figure 31: Range Compressed Signal vs. Azimuth Bin, $X n=60$

This waveform is the same shape as the one generated when the target was placed at the origin. The main lobe to side lobe distance is shorter than when at the origin, which is expected since the target is moving at $-30 \mathrm{~m} / \mathrm{s}$ in the azimuth direction.

Figure 32 shows the calculated peak distance for a stationary target as a function of the starting range position, from -10 to +10 range bins from the origin. This illustrates the effect that the three waveform shapes has on the algorithm. 


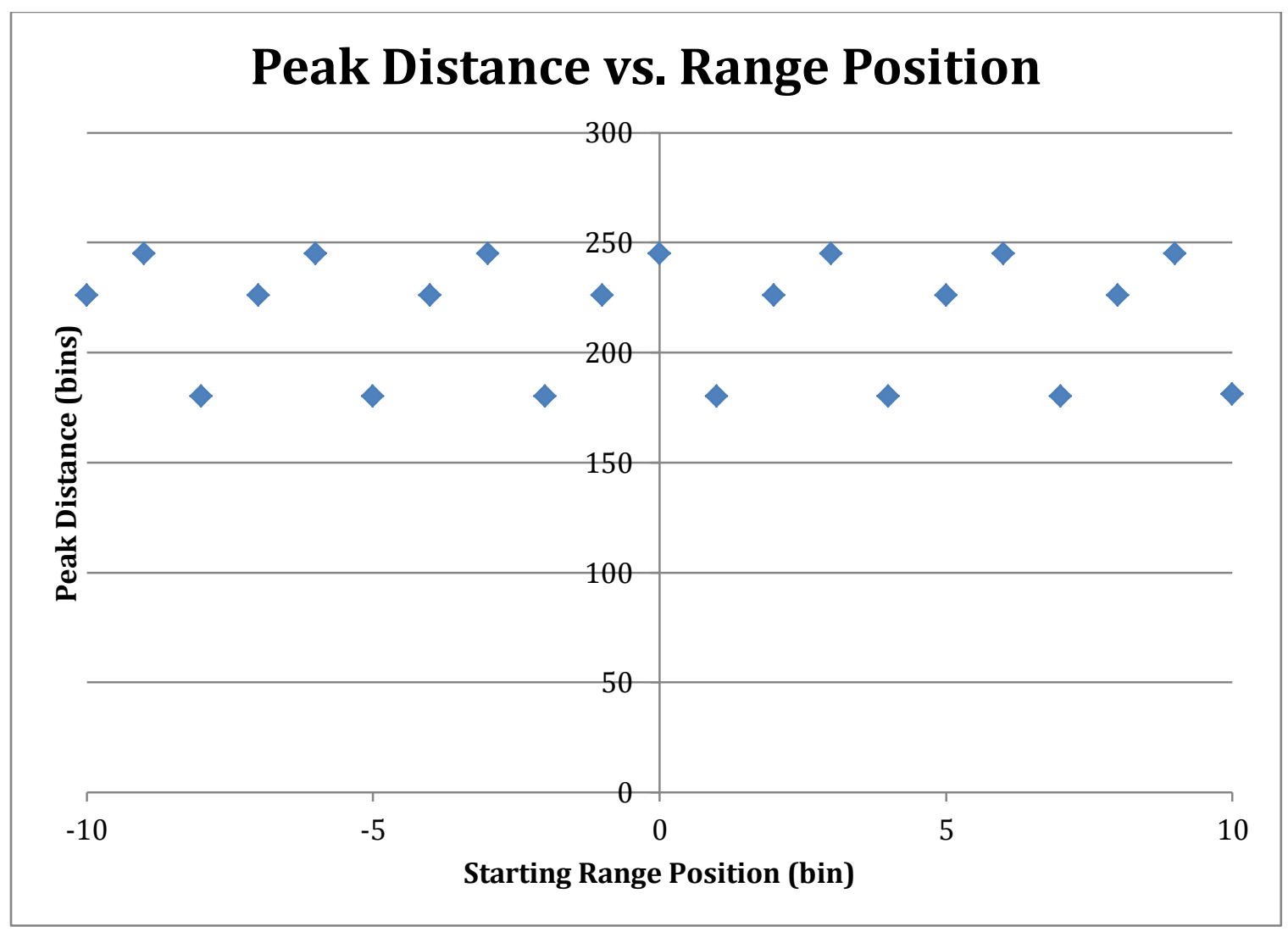

Figure 32: Measured Peak Distances vs. Starting Range Positions of Target

The peak distance is affected by the starting range position of the target. This causes the algorithm to determine the target's azimuth velocity incorrectly. 


\subsection{Range-Doppler Azimuth Velocity Detection Results}

As discussed in a previous section, the Range Doppler signal return of a target moving in the azimuth direction was characterized using two aspects: the peak value and the variance of the signal. Due to the nature of the Doppler Effect, we would expect a nonlinear correlation between the peak value and the target's azimuth velocity. In order to find the correlation between the target's azimuth velocity and the resulting RangeDoppler waveform, a point target was placed in the center of the target space. The target was then moved at a variety of azimuth velocities, and its Range-Doppler waveform was analyzed using MATLAB and its peak value recorded. This was implemented in code by comparing each range bin to find the local maximum in order to find the location of the streak in the range direction. Once the appropriate range was found, each azimuth bin at that range was analyzed to find the peak value. This allows the algorithm to accurately find the peak value if its location is not centered in the middle of the Range-Doppler plot due to the target's starting position.

Once the data was collected, the recorded peak values were plotted against the corresponding target azimuth velocity in Figure 33. 


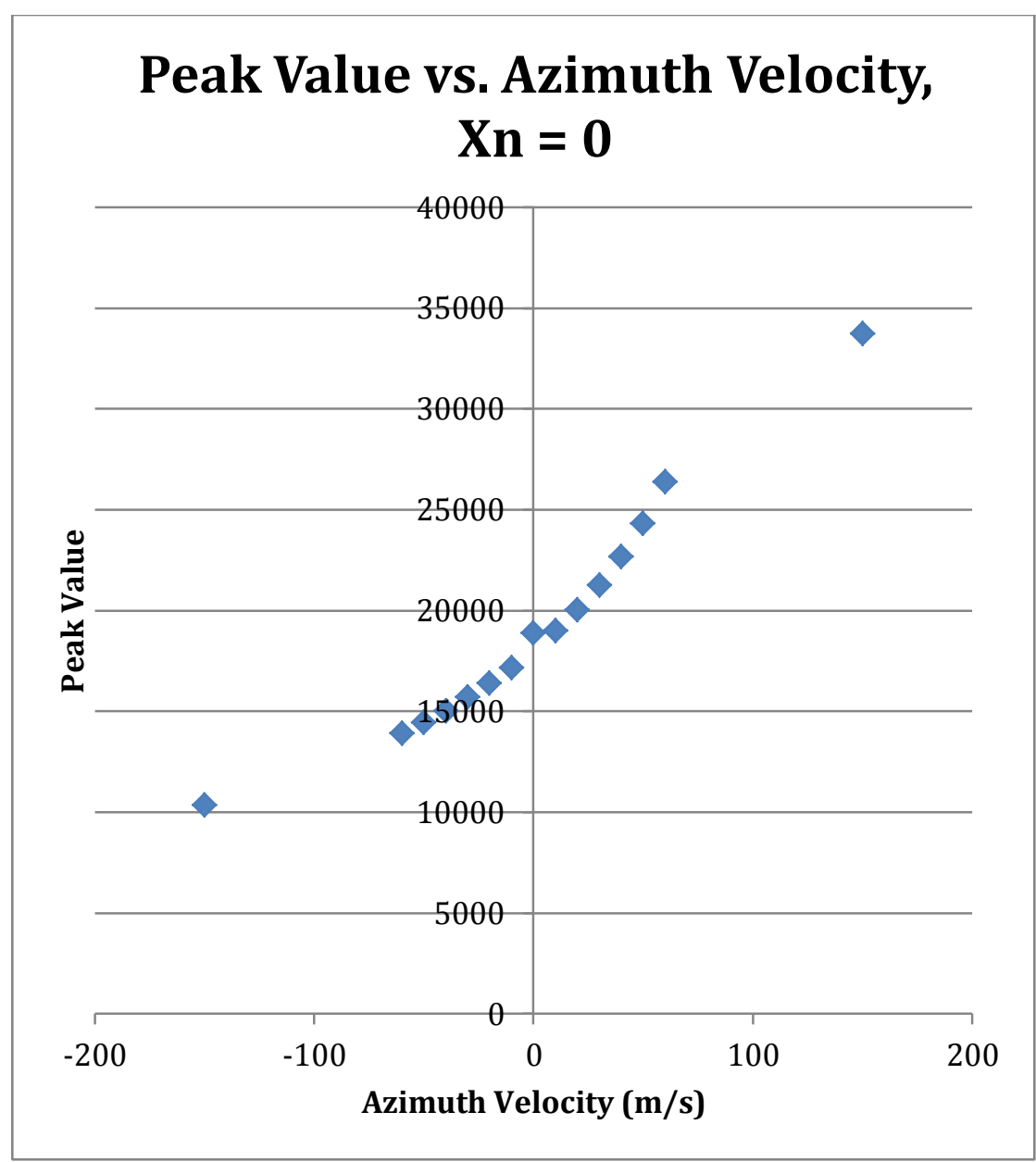

Figure 33: Range-Doppler Peak Values vs. Target Azimuth Velocities

From this data, it can be seen that the relationship between the Range-Doppler waveform's peak value and the target azimuth velocity is non-linear.

To determine the azimuth velocity that a particular peak value corresponds to, the inverse of Figure 33 was taken to show the azimuth velocity plotted against the peak value as Figure 34 . 


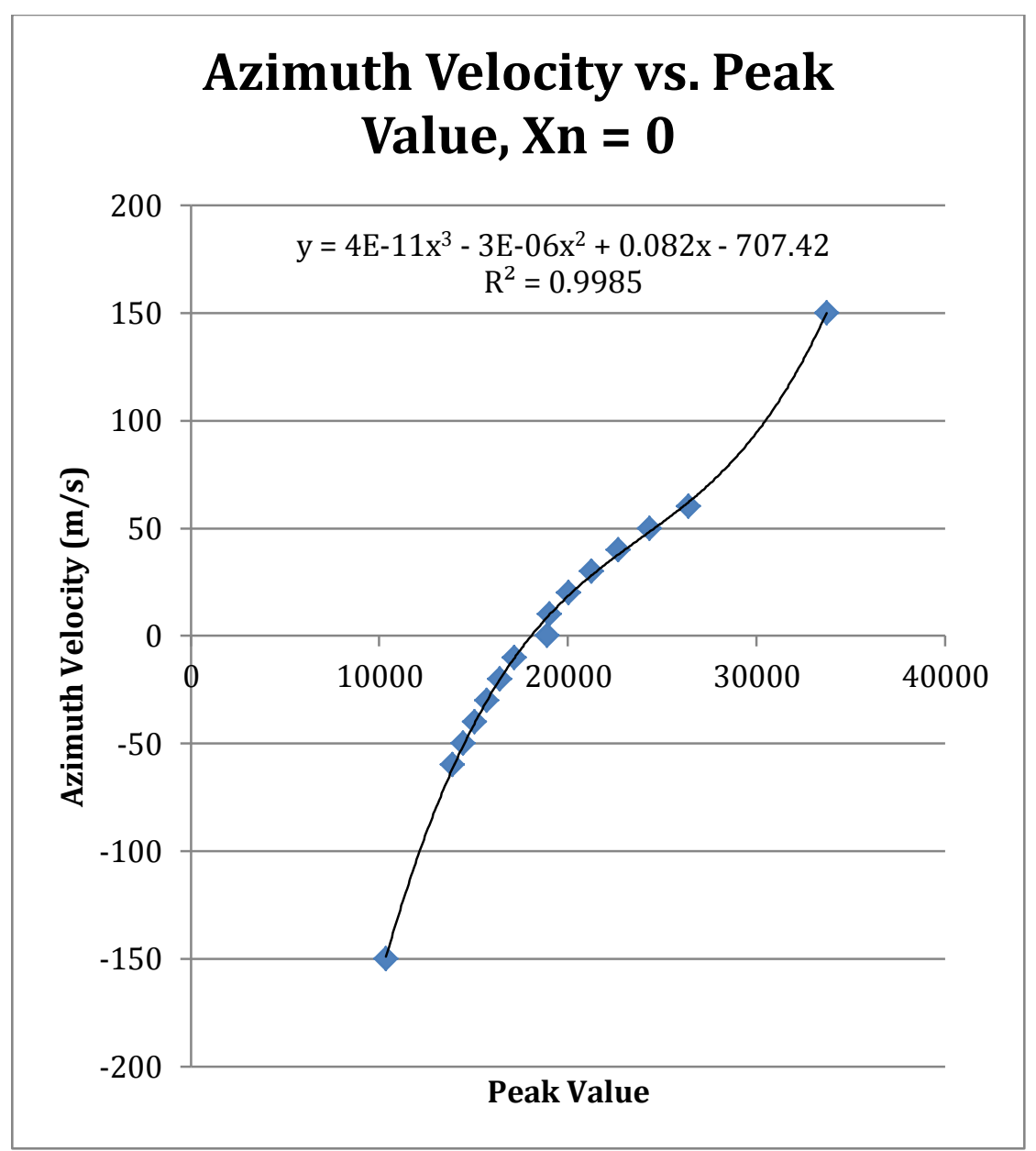

Figure 34: Target Azimuth Velocities vs. Range-Doppler Peak Values

Using the polyfit function in MATLAB on the above set of data results in Equation 15.

$$
v=4.0 * 10^{-11} x^{3}-3.0 * 10^{-6} x^{2}+0.082 x-707.42
$$

\section{Equation 15}

$v=$ estimated azimuth velocity

$x=$ peak value of the streak 
As seen in the Range Compressed azimuth velocity detection algorithm, the starting range location of the target affects the behavior of the target's signal return. Figure 35 is a graph of the peak value of the return signal as a function of the target's azimuth velocity.

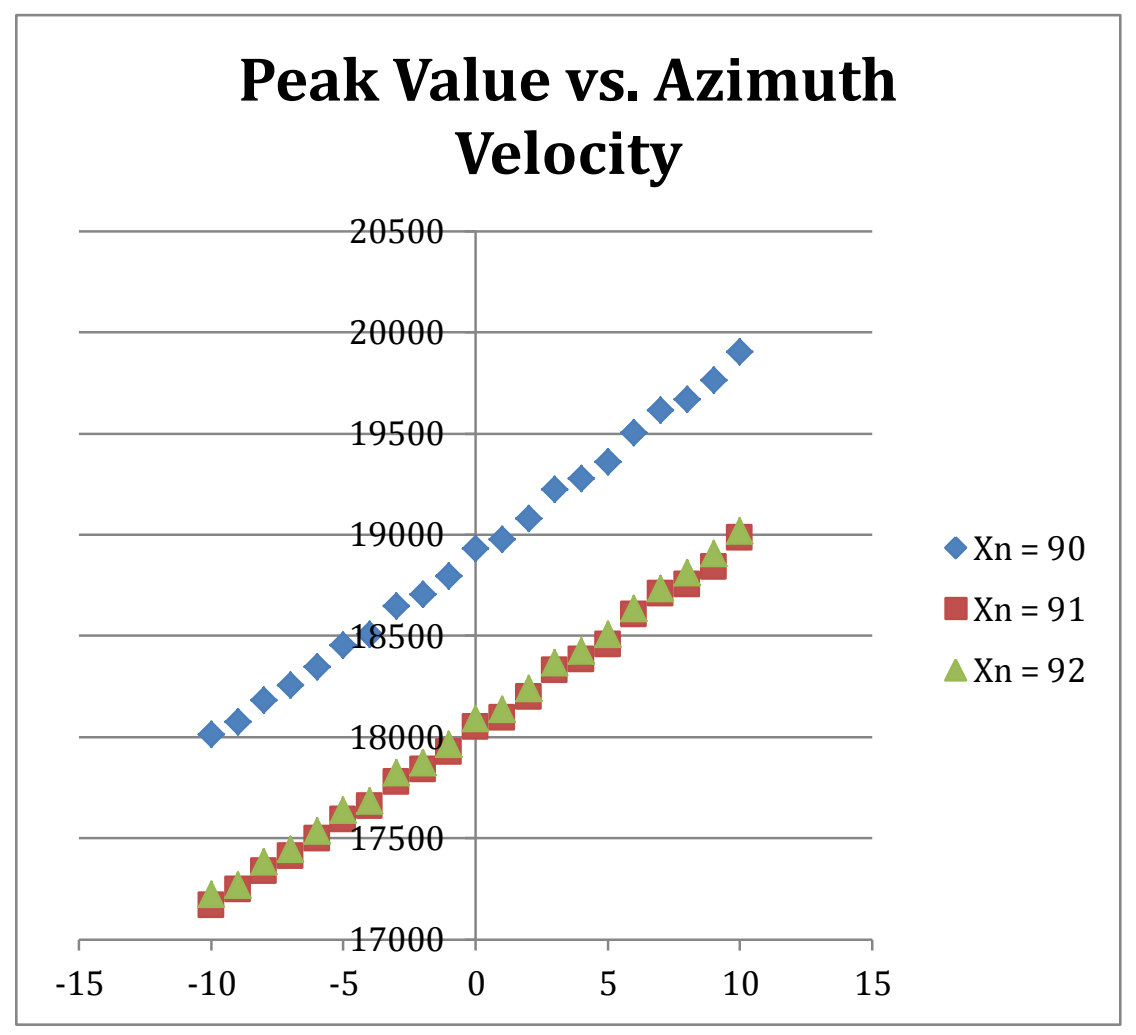

Figure 35: Range-Doppler Peak Values at Various Starting Range Positions

The different starting range positions of the target result in the peak value shifting significantly. A peak value of 19000 would correspond to an azimuth velocity of around $10 \mathrm{~m} / \mathrm{s}$ if the target started at range bin 91 or 92 , but only $1 \mathrm{~m} / \mathrm{s}$ if the target started at range bin 90 . This discrepancy significantly reduces the effectiveness of the velocity detection algorithm.

Another characteristic that can be used to distinguish the Range-Doppler streaks from each other is to compare the shape of the waveforms. A computationally efficient way to characterize this behavior is to take the standard distribution of each streak. Since a target 
moving with a negative azimuth velocity results in a smaller range of received frequencies, the shape of the waveform is compressed and with a tighter distribution. In contrast, a target moving with a positive velocity causes a larger range of received frequencies and a wider distribution. Therefore, the standard distribution of a particular streak should be enough information to determine the target's azimuth velocity.

Like the peak value measurements, the starting range location of the target affects the variance of the Range Doppler signal. The following are the three trends that seem to appear, depending on the starting range location of the target. A point target was placed in the target space, and its starting range position was varied from -40 to +40 , and the Range Doppler signal variance was plotted against the starting position. This experiment was performed 3 times, with the target having an azimuth velocity of $-30,0$, and $+30 \mathrm{~m} / \mathrm{s}$. Figure 36 shows the Range Doppler signal variances for various range starting positions when the target has an azimuth velocity of $-30 \mathrm{~m} / \mathrm{s}$.

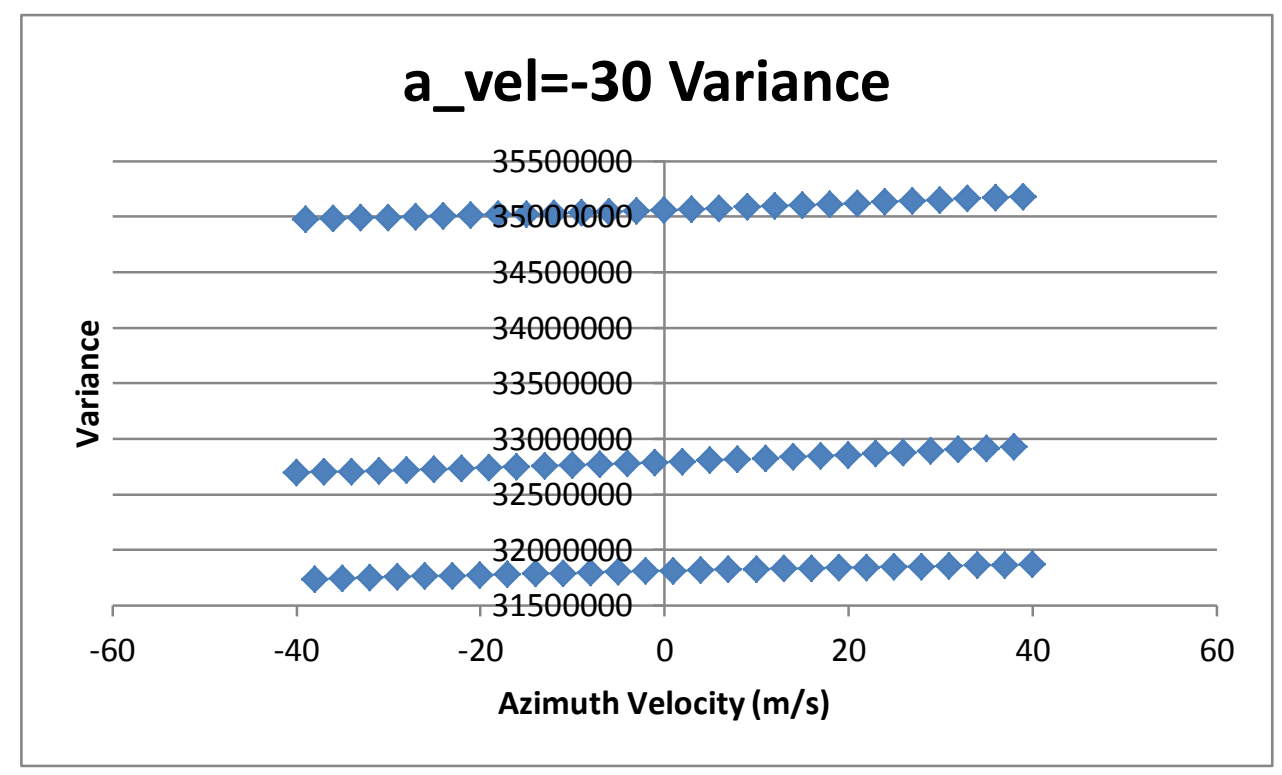

Figure 36: Signal Variance of Target With Azimuth Velocity of $-\mathbf{3 0} \mathbf{~ m} / \mathrm{s}$ 
The Range Doppler signal variances of a stationary target is shown as Figure 37.

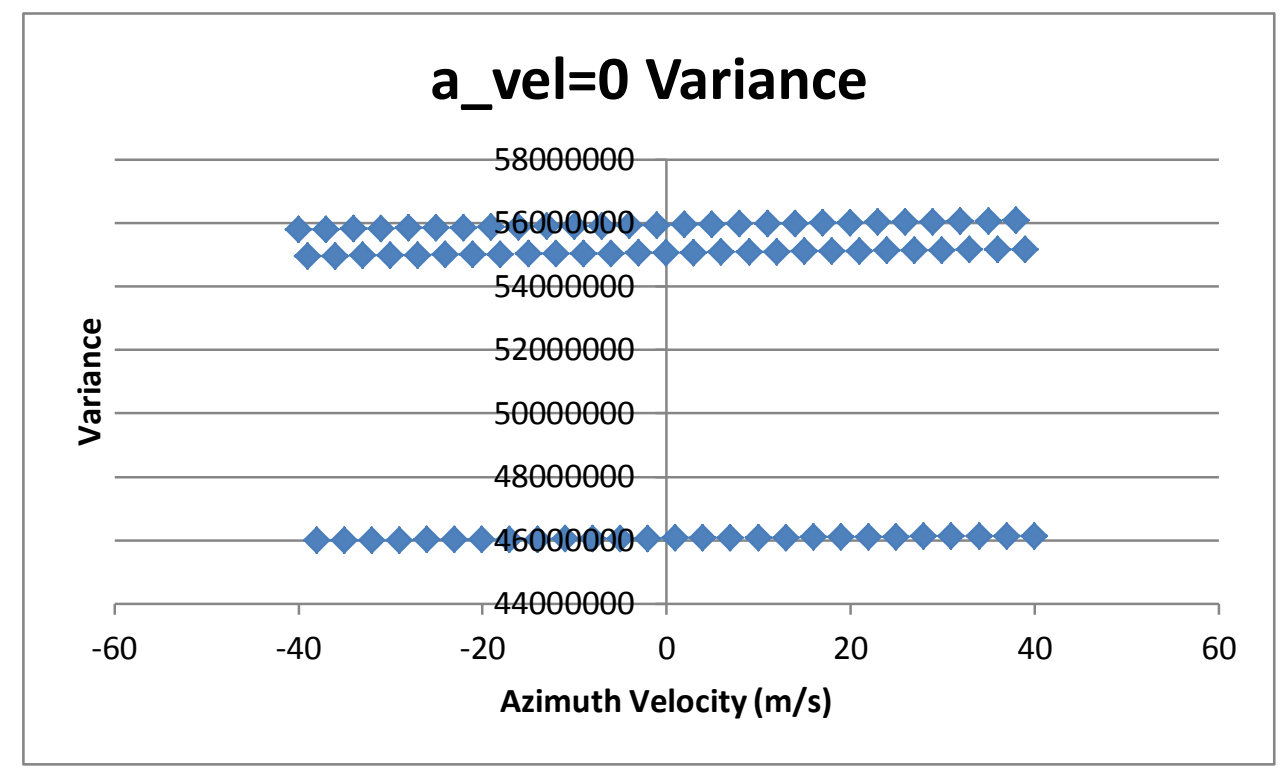

Figure 37: Signal Variance of Target With Azimuth Velocity of $0 \mathrm{~m} / \mathrm{s}$

Lastly, the Range Doppler signal variances of a target with an azimuth velocity of +30 $\mathrm{m} / \mathrm{s}$ is shown as Figure 38.

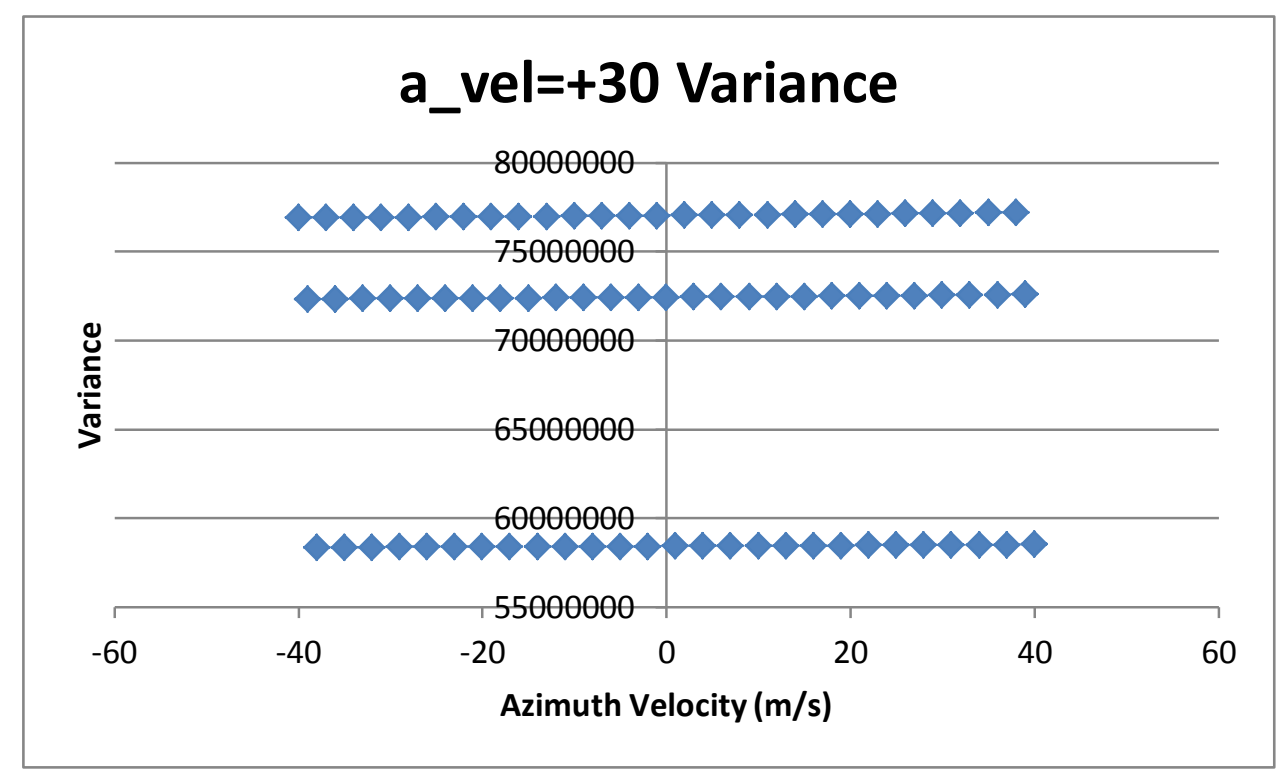

Figure 38: Signal Variance of Target With Azimuth Velocity of $+30 \mathrm{~m} / \mathrm{s}$ 
In all three cases, there appears to be 3 linear distributions that the variance values seem to lie on.

To characterize the variance of the target signal as the azimuth velocity changes, a point target was placed in the target space, and its azimuth velocity was changed from $-40 \mathrm{~m} / \mathrm{s}$ to $+40 \mathrm{~m} / \mathrm{s}$. The platform was moved across the target space each time, and the resulting Range Doppler signal returns were stored and analyzed. The variance of each signal was plotted against the target's azimuth velocity.

When the target is placed at $\mathrm{xn}=0$, the target is in the center of the target space. Figure 39 shows the effect the target's azimuth velocity has on the measured variance of the Range Doppler streak.

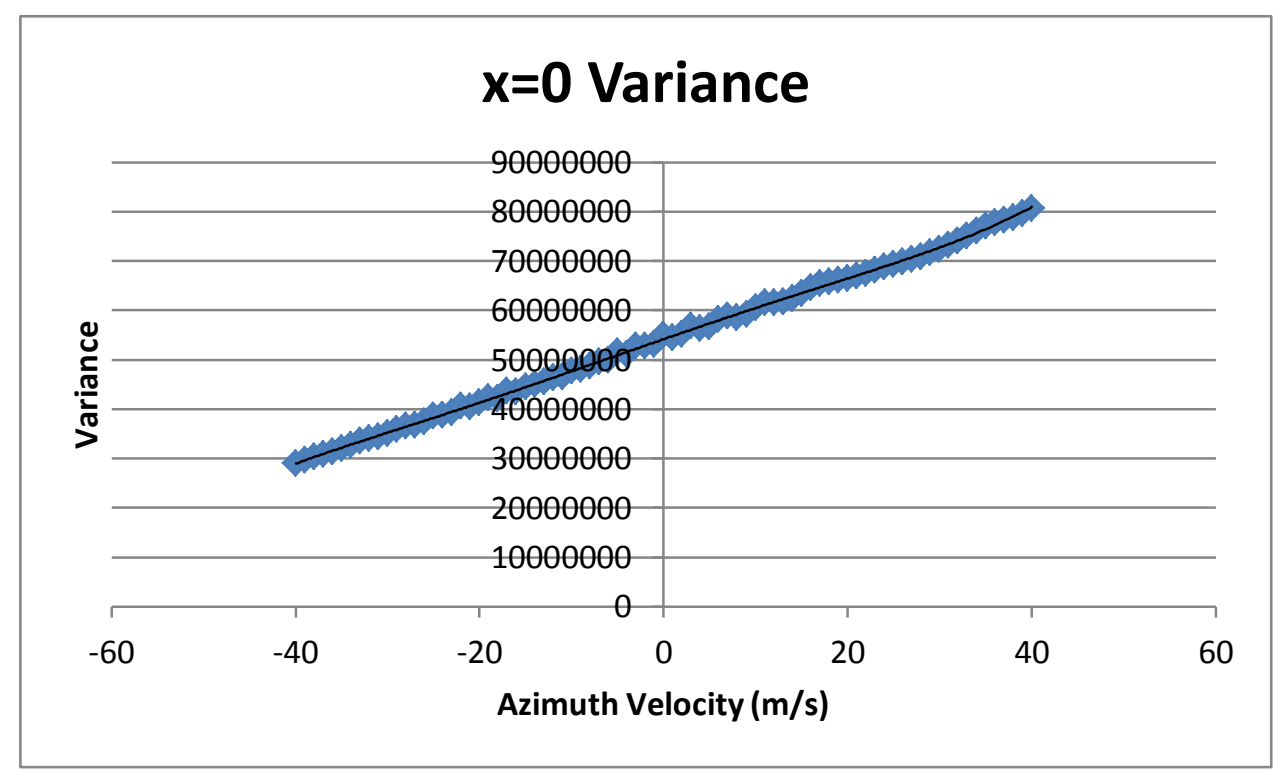

Figure 39: Signal Variance vs. Azimuth Velocity of Target Located At Xn = 0

Figure 40 is of the above data plotted as the measured variance versus the target's azimuth velocity. 


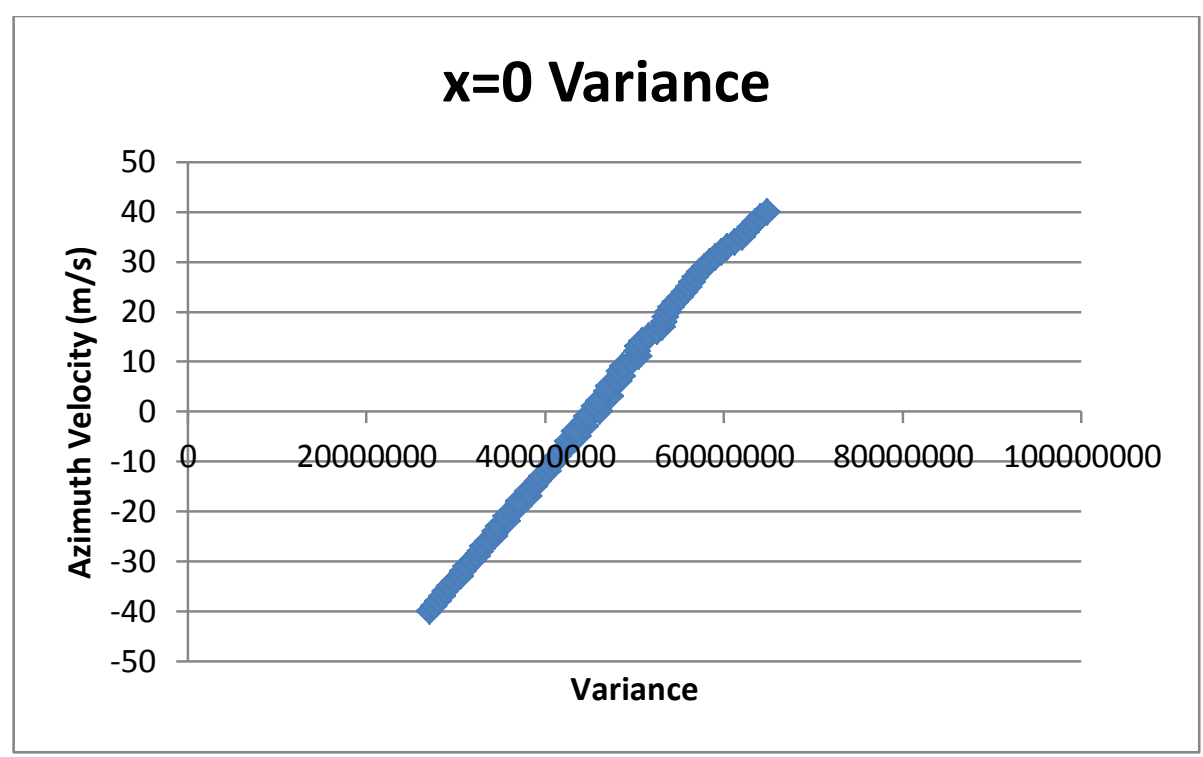

Figure 40: Azimuth Velocity vs. Signal Variance of Target Located At Xn = 0

The linear fit to this graph, as calculated by the MATLAB function polyfit, is shown as Equation 16.

$$
v=1.58 * 10^{-6} x-85.3633
$$

\section{Equation 16}

$\mathrm{v}=$ estimated azimuth velocity

$\mathrm{x}=$ variance

The second general trend occurs when the starting range location of the target is at $\mathrm{xn}=1$. Because of this change, the target has different Range Doppler signal variances for the same azimuth velocity, as shown in Figure 41. 


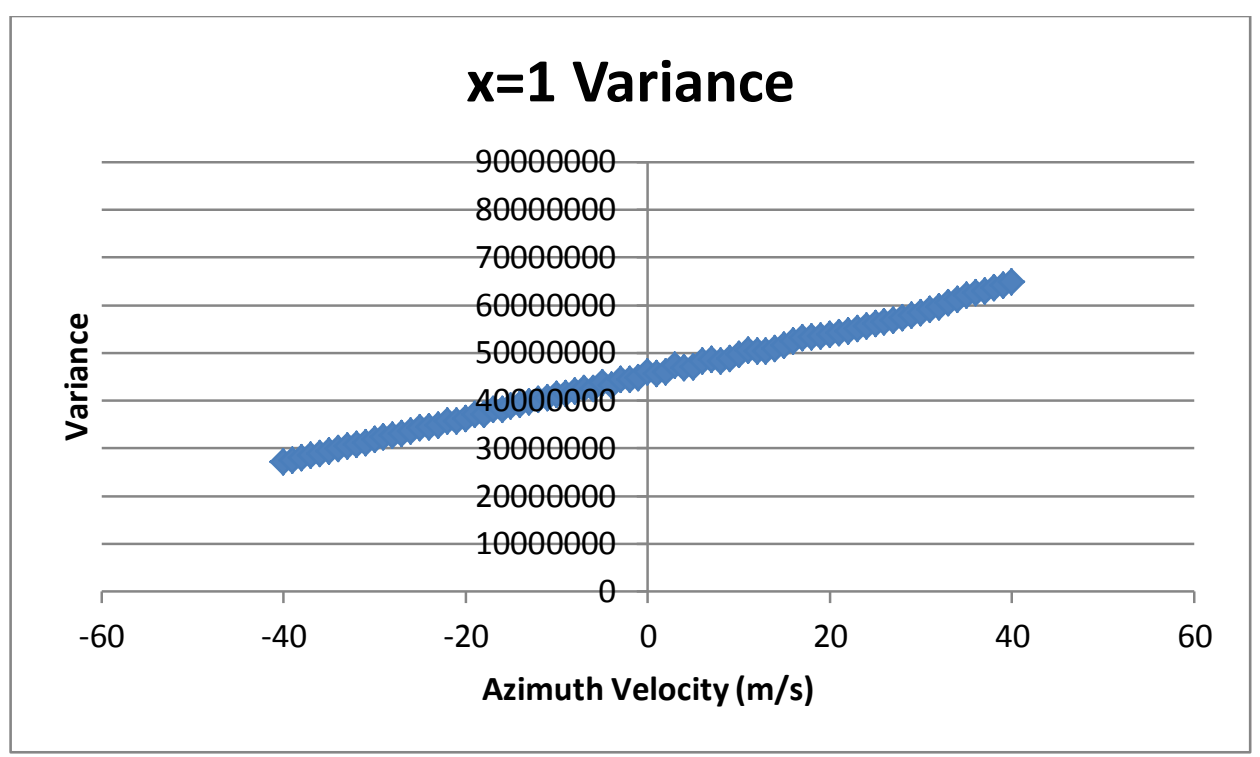

Figure 41: Signal Variance vs. Azimuth Velocity of Target Located At Xn = 1

The target's azimuth velocity as a function of the Range Doppler signal variance is shown in Figure 42.

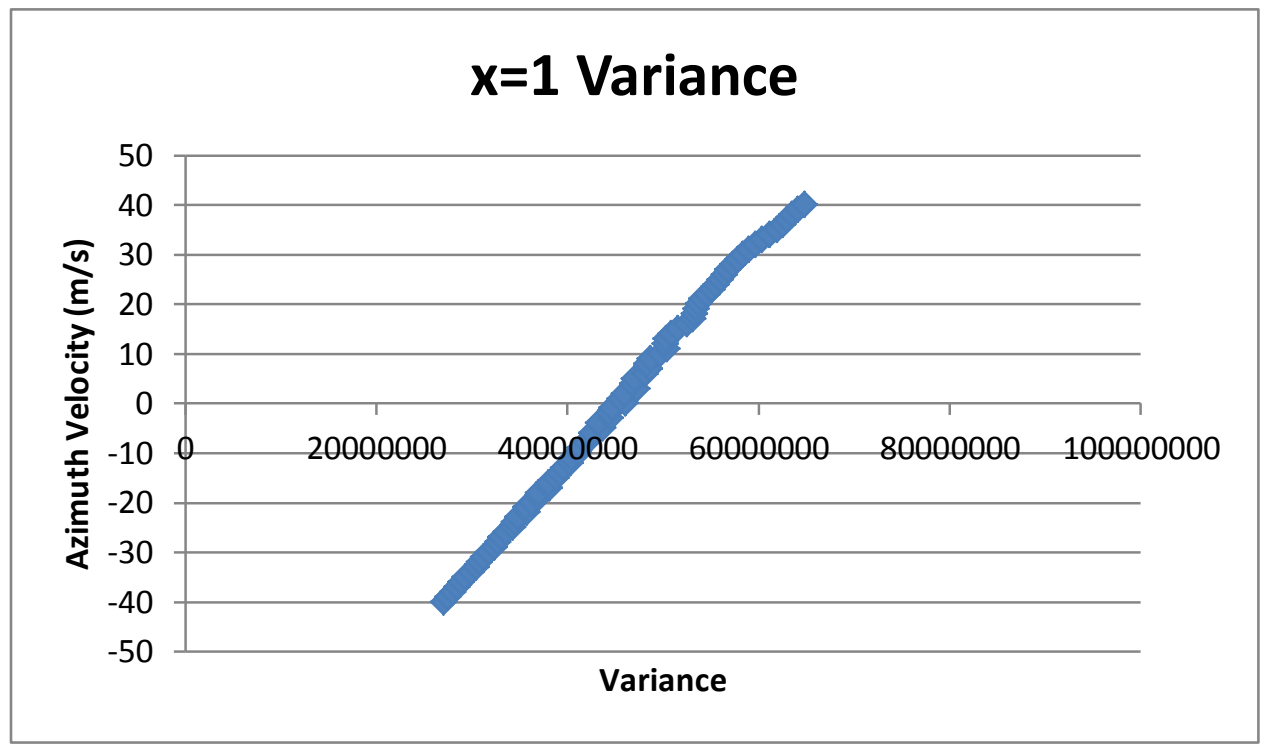

Figure 42: Azimuth Velocity vs. Signal Variance of Target Located At Xn = 1

When processed using polyfit, the linear fit equation for this relation ship is shown as Equation 17. 


$$
v=2.21 * 10^{-6} x-100.05
$$

\section{Equation 17}

$\mathrm{v}=$ estimated azimuth velocity

$\mathrm{x}=$ variance

The third general trend of Range Doppler signal variances can be seen when the target is placed at the range location $\mathrm{xn}=2$. With this configuration, the effect on the measured variance is shown as Figure 43.

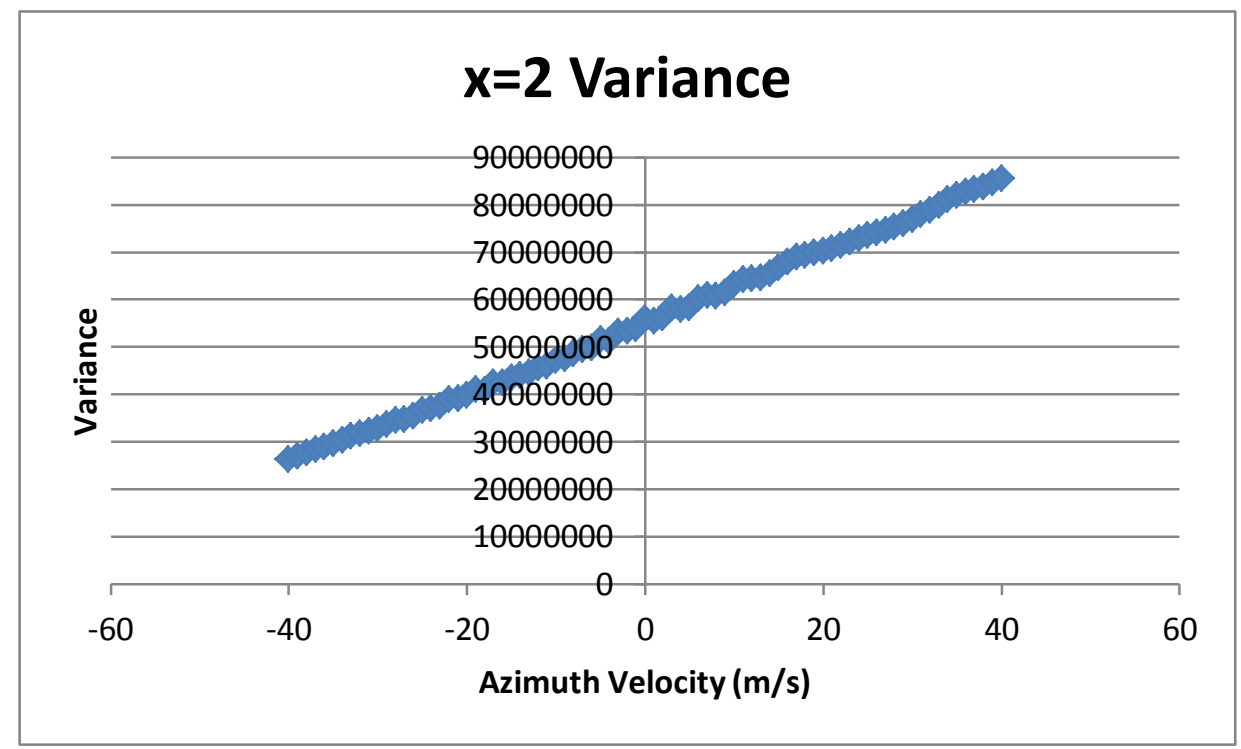

Figure 43: Signal Variance vs. Azimuth Velocity of Target Located At Xn $=2$

Again, the target's azimuth velocity is represented as a function of the measured Range Doppler signal variance, which is shown in Figure 44. 


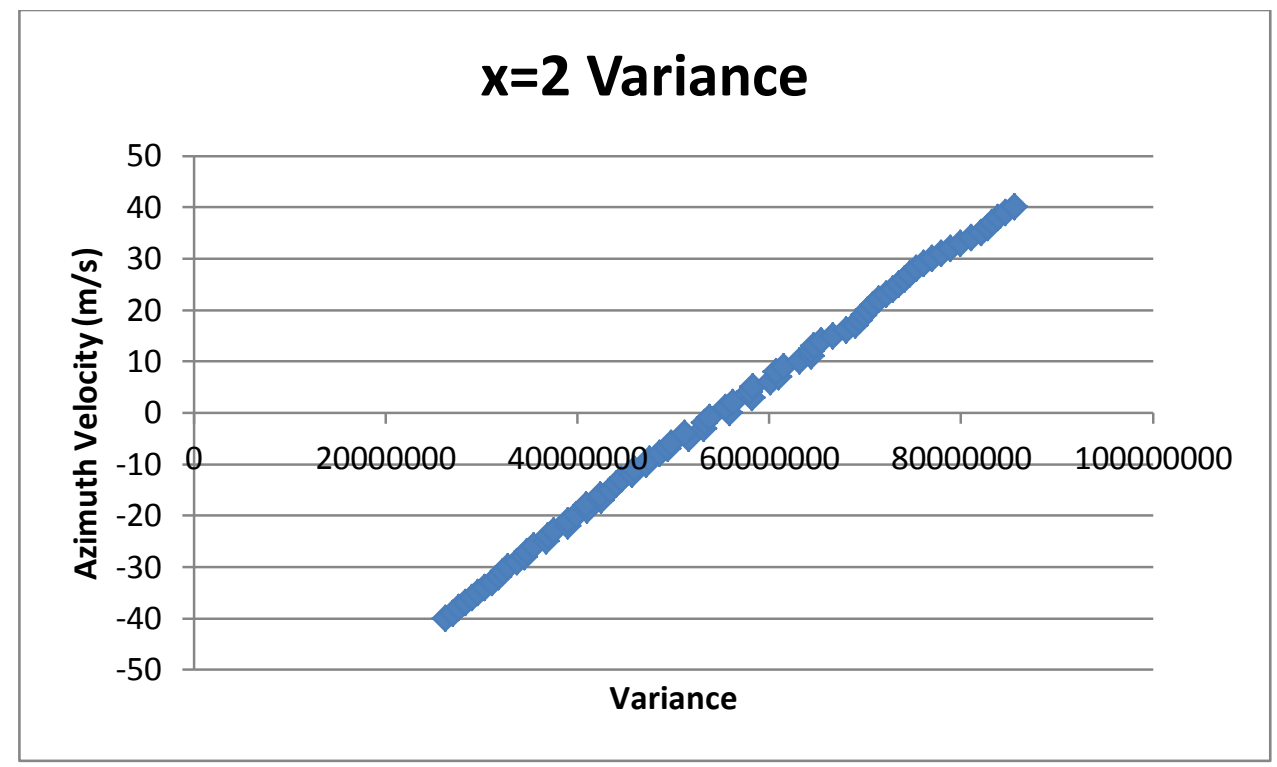

Figure 44: Azimuth Velocity vs. Signal Variance of Target Located At Xn = 2

The MATLAB function polyfit calculates the best-fit linear function of this set of data as Equation 18.

$$
v=1.34 * 10^{-6} x-73.8958
$$

\section{Equation 18}

$\mathrm{v}=$ estimated azimuth velocity

$\mathrm{x}=$ variance

To evaluate the performance of the azimuth velocity detection algorithm, the error between the target's azimuth velocity and the best-fit equation was plotted against the azimuth velocity. Each of the three equations were plotted separately to show the individual behavior of each best-fit line. Figures 45 and 46 show the absolute and percent error, respectively, that is seen at $\mathrm{xn}=0$. 


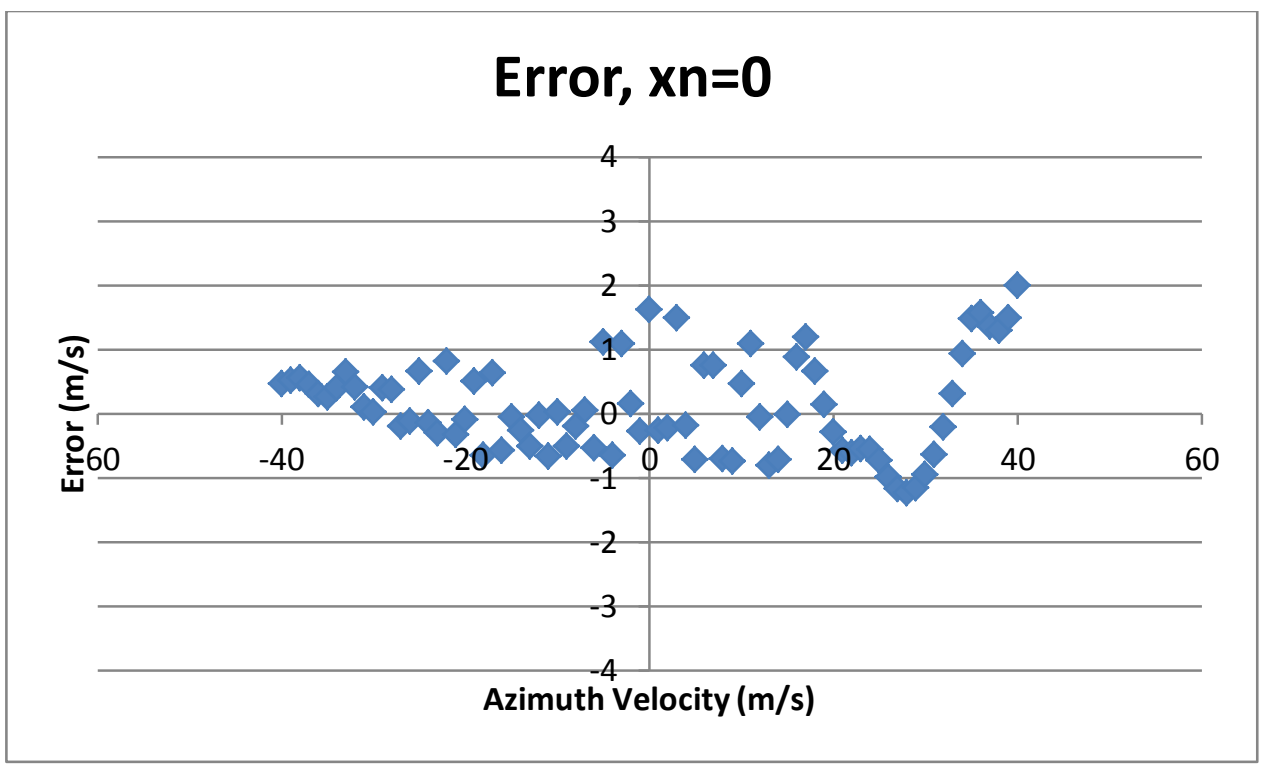

Figure 45: Absolute Error of Azimuth Velocities of Target Located at Xn = 0

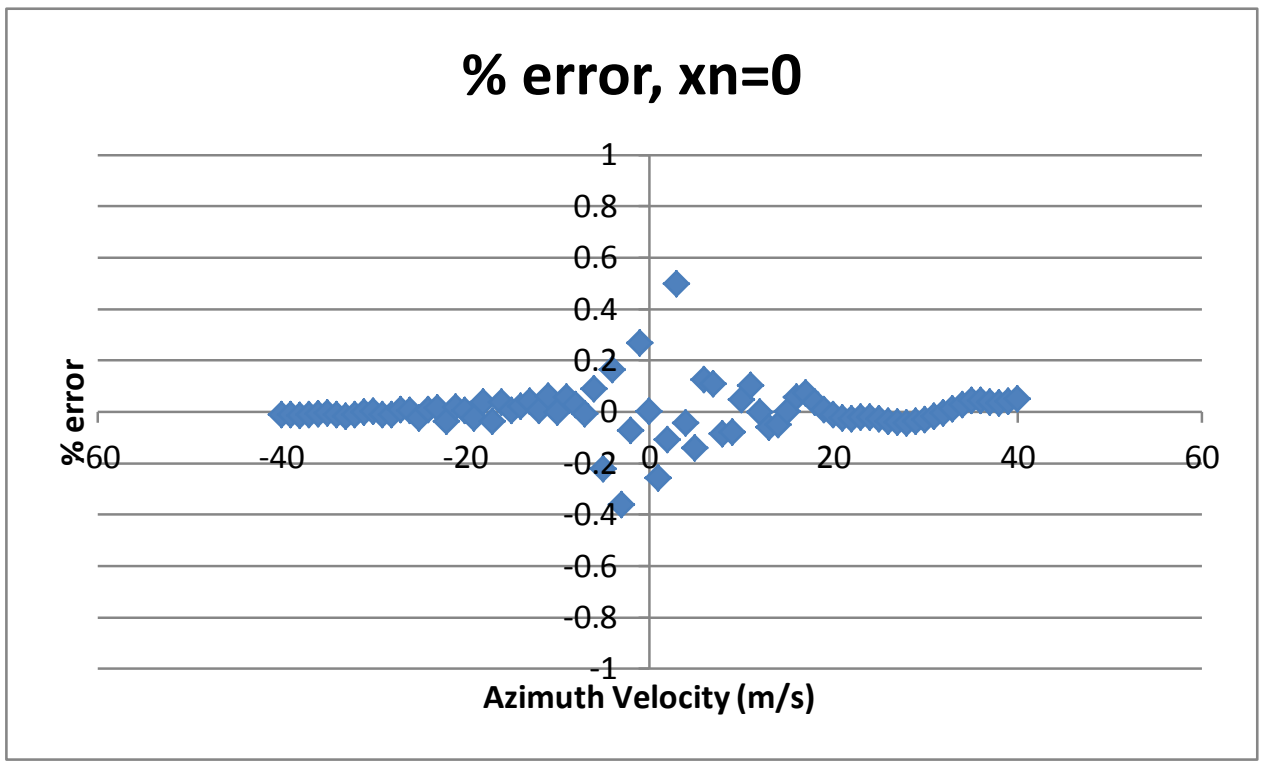

Figure 46: Percent Error of Azimuth Velocities of Target Located at Xn = 0

Figures 47 and 48 show the azimuth velocity error of a target placed at $x n=1$ at the beginning of the simulation. 


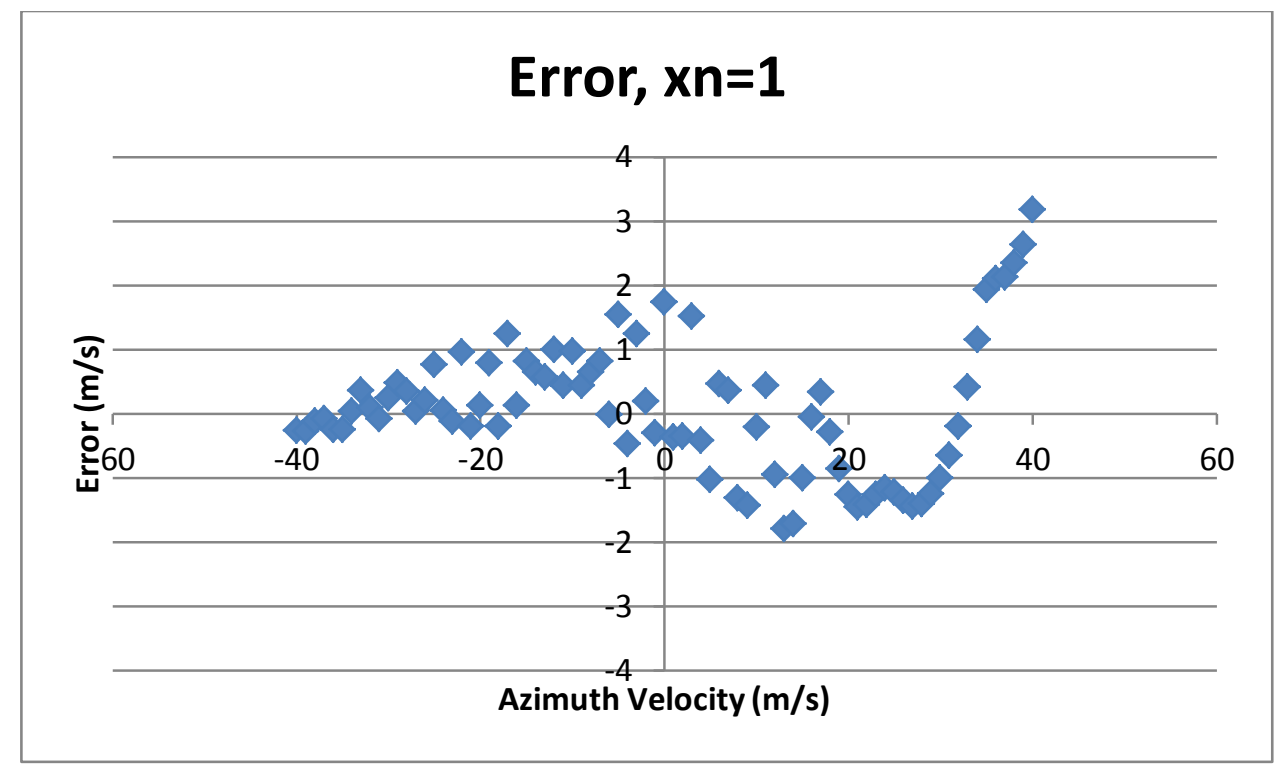

Figure 47: Absolute Error of Azimuth Velocities of Target Located at Xn = 1

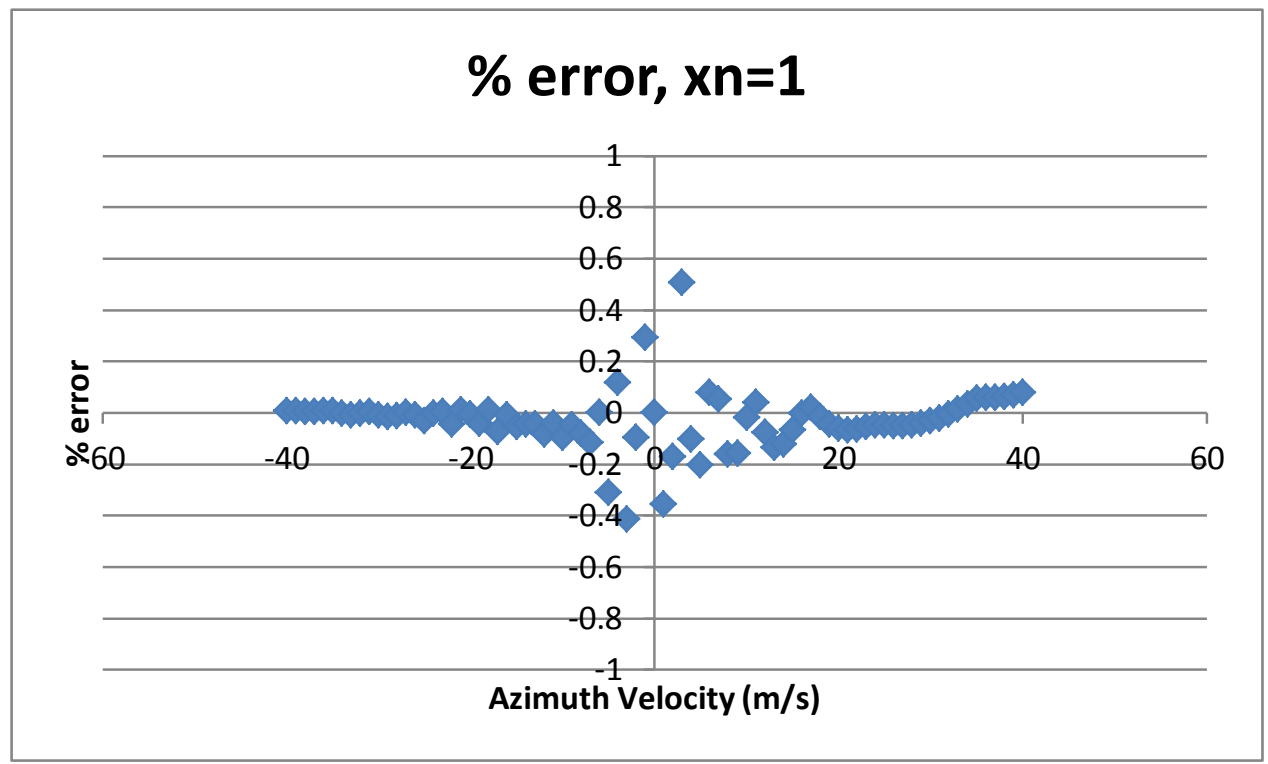

Figure 48: Percent Error of Azimuth Velocities of Target Located at Xn = 1

The error graphs for a target placed $\mathrm{xn}=2$ is shown as Figures 49 and 50 . 


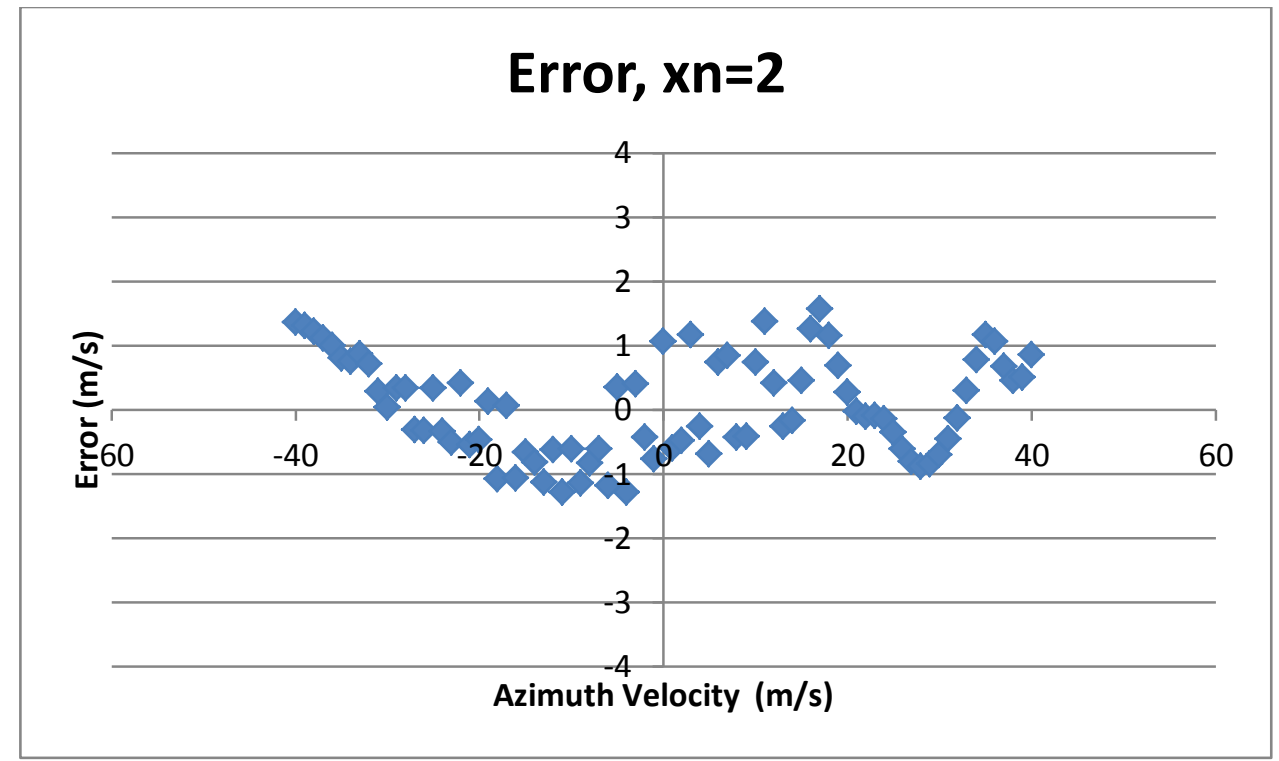

Figure 49: Absolute Error of Azimuth Velocities of Target Located at Xn = 2

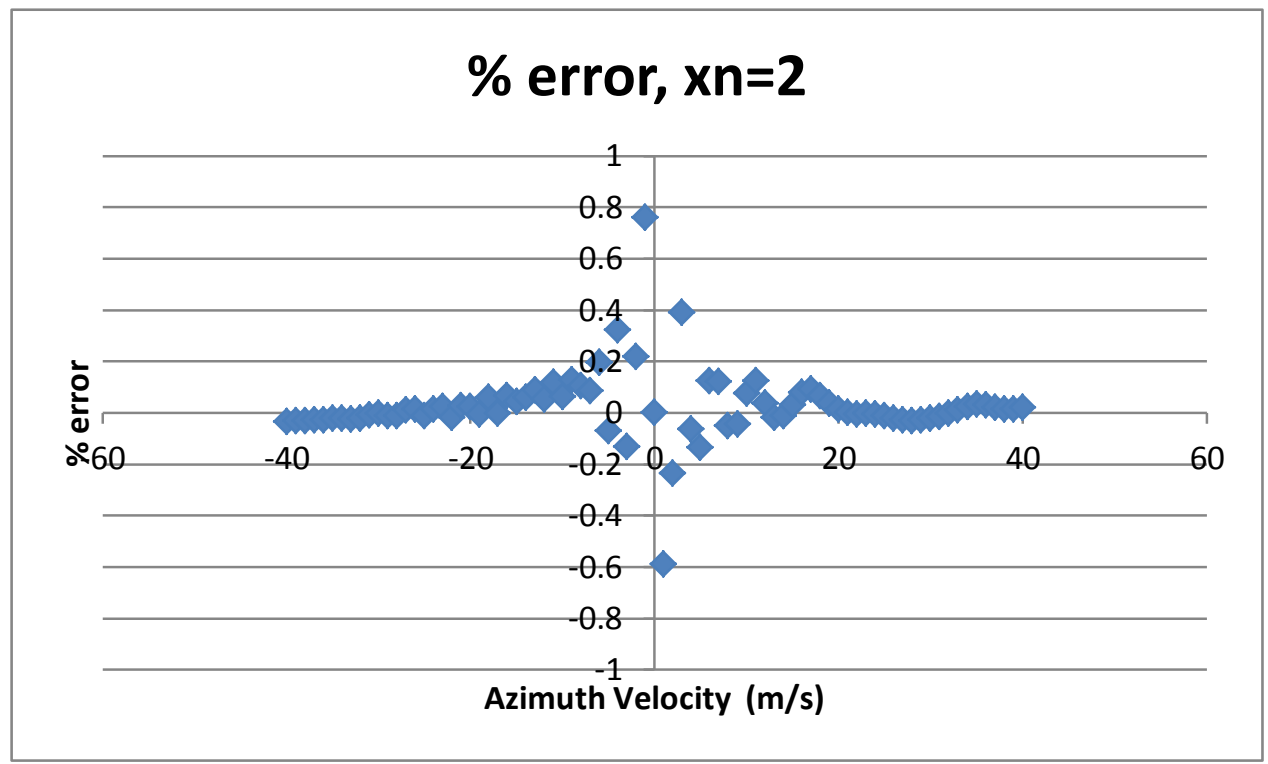

Figure 50: Percent Error of Azimuth Velocities of Target Located at Xn $=2$

From the above results, all three of the equations result in a similar \% error in the reported target azimuth velocity. There appears to be a $+/-2 \mathrm{~m} / \mathrm{s}$ error in the result, irrespective of the actual azimuth velocity. Therefore, the $\%$ error for the target moving at low azimuth velocities are much higher than when the target is moving at more than 15 $\mathrm{m} / \mathrm{s}$. 


\section{CONCLUSION / FUTURE WORK}

This paper explored several different approaches in determining the range and azimuth velocities of a point target in the early stages of the SAR signal processing. These strategies can reduce the computation time needed to determine the velocity of the point target.

The range velocity of a moving target is apparent by analyzing the Range Compressed signal returns, which is generated in the first step of the SAR processing algorithm. The slope of the return signature is proportional to the velocity of the target, and by calculating the slope accurately, the target velocity can be quickly determined with a percent error of less than $2 \%$ for the data set under test.

A moving target's azimuth velocity was characterized using multiple approaches. The first detection algorithm was done in the Range Compressed space, and attempted to correlate the distance between the main lobe and side lobes of the target at a single range bin. In the Range Doppler space, the signal return from the target could be used to get information about the azimuth velocity as well. The peak value and variance of the returned signal were two aspects that were investigated, and their behavior as the target azimuth velocity changes was characterized. Best-fit curves for all three algorithms were determined based on experimental results, and the target's azimuth velocity could be reliably determined when the target was located in a specific position. However, it was found that the starting range location (xn) of the target affected the values of the side lobe to main lobe distance in the Range Compressed signal, as well as the peak value and variance in the Range Doppler signal. In the case of the variance behavior, three different 
linear fit equations were used to more accurately determine the target's azimuth velocity at all starting range locations.

There are some opportunities for further work in range and azimuth velocity rapid detection. This paper focused on point targets moving in a constant velocity in the target space. Some research can be done on how a multi-point target moving in the range and azimuth direction will appear in the various stages of SAR processing, and if sufficient inforamtion can be gleaned from the intermediate signals to reliably determine the velocity of the target. In addition, this paper does not cover targets accelerating in any direction. One first step could be to investigate how a point target accelerating in the same direction as it is moving will affect the returned signal. This experiment could be expanded to include units changing its heading within the duration of the SAR sampling. An interesting problem would also be to analyze the effectiveness of each of these algorithms if the target disappears from the target space for a period of time due to camoflauge, the intersection of multiple units, or obstruction of view. 


\section{BIBLIOGRAPHY}

1) C. Yang, et al, "Velocity estimation of moving targets on the sea surface by azimuth differentials of simulated-SAR image," Geoscience and Remote Sensing Symposium,2009 IEEE International,IGARSS 2009 , vol.3, no., pp.III-809,III-812, $12-17,2009$

2) C. Yang, et al, "A simple maneuver indicator from target's range-doppler image," Information Fusion, 2007 10th International Conference on Digital Object Identifier, pp.1,8, 9-12, 2007

3) Yue-Feng Tan; Zhu Xueyong; Wen-Qin Wang, "Impacts of velocity deviation on spaceborne high-resolution DPCA SAR," Computational Problem-Solving (ICCP), 2010 International Conference on, vol., no., pp.442,445, 3-5 Dec. 2010

4) Radius, A.; Solimini, D., "A velocity vector estimation algorithm tested on simulated SAR raw data," Geoscience and Remote Sensing Symposium, 2007. IGARSS 2007. IEEE International, vol., no., pp.563,566, 23-28 July 2007

5) Fienup, J.R., "Detecting moving targets in SAR imagery by focusing," Aerospace and Electronic Systems, IEEE Transactions on , vol.37, no.3, pp.794,809, July 2001

6) Kawalec, A.; Serafin, P., "Estimation Of Target Movement Parameters In Synthetic Aperture Radar," Radar Symposium, 2006. IRS 2006. International, vol., no., pp.1,4, 24-26 May 2006 
7) B. Mahafza and A. Elsherbeni, MATLAB Simulations for Radar Systems Design. Boca Raton, FL: CRC/Chapman \& Hall, 2004.

8) G. Stimson, Introduction to Airborne Radar. Stevenage: IEEE, 1998.

9) M. Connor, et al, "Multiple Target Tracking," Worchester Polytechnic Institute, Worchester, NY, 2008.

10) G. Newstadt, et al, "Detection/Tracking of moving targets with synthetic aperture radars,” Dept. Elect. Comput. Sci., Univ. Michigan, Michigan, 2010.

11) M. Schlutz, "Synthetic Aperture Radar Imaging Simulated in Matlab," M.S. thesis, Dept. Elect. Eng., California Polytechnic State Univ., San Luis Obispo, 2009.

12) M. Soumekh, Synthetic Aperture Radar Signal Processing with MATLAB Algorithms. New York, NY: Wiley \& Sons, Inc., 1999.

13) I. Cumming, et al, Digital Processing of Synthetic Aperture Radar Data: Algorithms and Implementations. Norwood, MA : Artech House, Inc., 2005.

14) Saghri, John A. Private conversations. 


\section{APPENDIX A: MATLAB CODE USER GUIDE}

\section{$\underline{\text { Overview }}$}

There are two MATLAB files used to run simulations as presented in the paper. Moving_vs_stationary. $m$ is the simulation file that creates multiple point targets, simulates Synthetic Aperture Radar samples, and then applies the various velocity detection algorithms in an attempt to accurate determine the range and azimuth velocities of the target. Constant_azimuth_velocity.m runs the same set of simulations, but allows multiple iterations of the simulation to be run, modifying system parameters each time. This allows trends across multiple velocities or starting positions to be characterized. Ftx.m, fty.m, iftx.m, and ifty.m are MATLAB functions that perform FFTs and inverse FFTs on the azimuth and range bins, which are required to generate the SAR images. The simulation environment included in the MATLAB files included with this document was developed by Brian Zaharris. The code snippets shown in sections Determining Range Velocity, Determining Azimuth Velocity with the Range-Compressed Signal, and Determining Azimuth Velocity with the Range-Doppler Signal are my contributions to the MATLAB functions. 


\section{Parameter Setup}

The following lines of code establish the various parameters used in the simulation:

$\%$ Tracking Parameters

X_movement $=0$;

y_movement $=0$;

$\%$ General Parameters

focus $=1 ; \quad \%$ Set this flag to focus moving targets

track $=1 ; \quad \%$ Set this flag to track moving targets

noise $=0 ; \quad \%$ Set this flag to add noise to signal

ntarget $=1 ; \quad \%$ Set the number of targets

$\mathrm{cj}=\operatorname{sqrt}(-1)$;

pi2 $=2 *$ pi;

$\mathrm{c}=3 \mathrm{e} 8 ; \quad \%$ Propagation speed

$\mathrm{fc}=4.5 \mathrm{e} 9 ; \quad \%$ Carrier frequency $(4.5 \mathrm{GHz})$

$\mathrm{wc}=\mathrm{pi} 2 * \mathrm{fc}$;

lambda=c/fc; $\quad \%$ Wavelength $(60 \mathrm{~cm})$

$\mathrm{k}=\mathrm{pi} 2 / \mathrm{lambda} ; \quad \%$ Wavenumber

$\mathrm{vp}=200 ; \quad \%$ Velocity of platform

$\mathrm{PRF}=300 ; \quad \%$ Pulse repitition frequency

dur=30; $\quad \%$ Time of Flight

eta=linspace(0,dur,PRF*dur); \% Slow Time Array

eta $=$ transpose $($ eta);

std_dev=.75; $\quad \%$ Standard Deviation of Noise

$\mathrm{Fn}=1 ; \quad \%$ Reflectivity

$\%$ range parameters

$\mathrm{Xc}=20000 ; \quad \%$ Range distance to center of target area

$\mathrm{X} 0=200 ; \quad \%$ Target area in range is within [Xc-X0, $\mathrm{Xc}+\mathrm{X} 0$ ]

$\mathrm{Tp}=.25 \mathrm{e}-5 ; \quad \%$ Chirp pulse duration;

B0=100e6; $\quad \%$ Baseband bandwidth is plus/minus B0

alpha=B0/Tp; $\quad \%$ Range Chirp Rate

$\%$

$\mathrm{dx}=\mathrm{c} /(2 * \mathrm{~B} 0) ; \quad \%$ Range resolution $(1.5 \mathrm{~m})$

$\mathrm{dt}=1 /(2 * \mathrm{~B} 0) ; \quad \%$ Time Domain Sampling Interval

$\%$

$\mathrm{rsr}=1 / \mathrm{dt} ; \quad \%$ Range Sampling Rate

$\mathrm{Ts}=(2 *(\mathrm{Xc}-\mathrm{X} 0)) / \mathrm{c} ; \quad \%$ Start time of sampling

$\mathrm{Tf}=(2 *(\mathrm{Xc}+\mathrm{X} 0)) / \mathrm{c}+\mathrm{Tp} ; \quad \%$ End time of sampling

$\%$ Azimuth Parameters

$\mathrm{La}=2 ; \quad \%$ Actual length of Antenna 


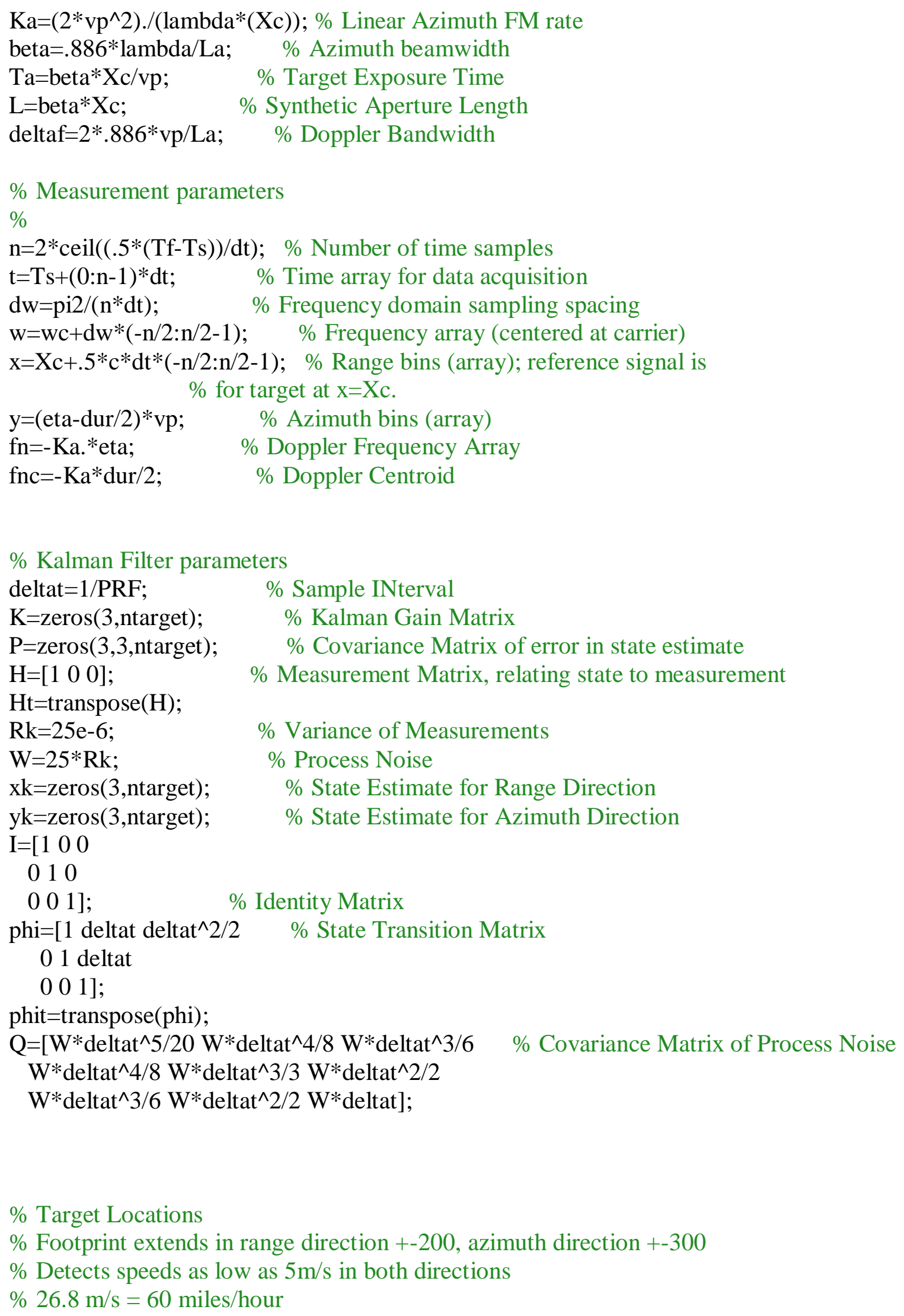




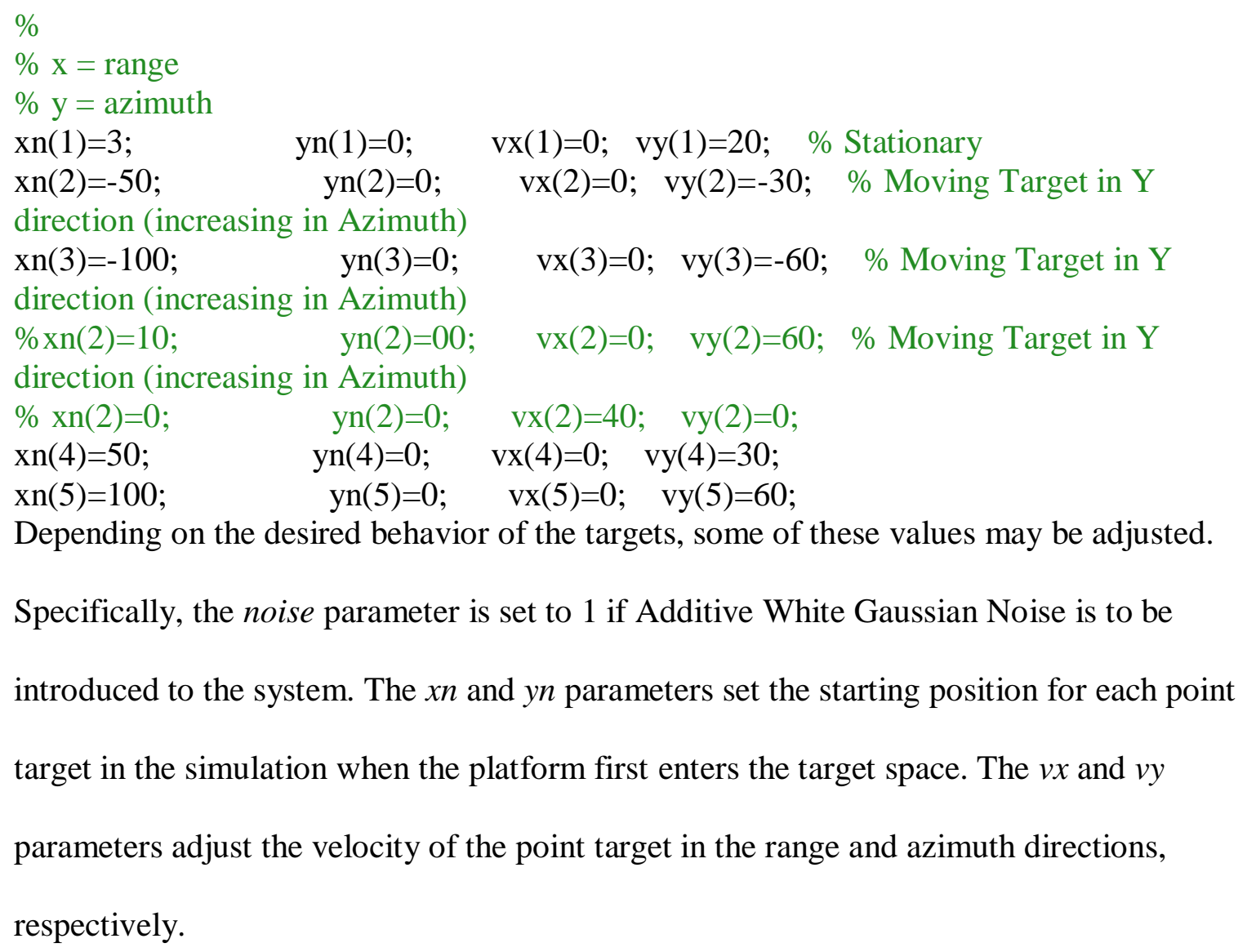




\section{Determining Range Velocity}

The range velocity is determined using the following MATLAB code:

if $\left(\mathrm{x} \_\right.$movement $\left.==1\right)$

$\%$ X Velocity Calculations

$\%$ Determine values at center of line, offsets from center

xcenter_index $=$ ceil $\left(\mathrm{PRF}^{*}\right.$ dur/2);

[xcenter_value, $x$ center $]=\max ((\operatorname{smb}($ xcenter_index,$:)))$;

xcenter

$\%$ xcenter

$\%$ Find values at $+/-100,200$ from center

xcenter_left_100_index $=$ ceil $\left(\mathrm{PRF}^{*}\right.$ dur/2)-100;

[xcenter_left_100_value,xcenter_left_100] $=\max ((\operatorname{smb}($ xcenter_left_100_index,:) $))$;

xcenter_right_100_index $=$ ceil $\left(\mathrm{PRF}^{*} \mathrm{dur} / 2\right)+100$;

[xcenter_right_100_value,xcenter_right_100] =

$\max \left(\left(\operatorname{smb}\left(x c e n t e r \_r i g h t \_100 \_i n d e x,:\right)\right)\right)$;

$\%$ xcenter_left_100

$\%$ xcenter_right_100

xcenter_left_200_index $=$ ceil $\left(\mathrm{PRF}^{*}\right.$ dur/2)-200;

[xcenter_left_200_value,xcenter_left_200] $=\max ((\operatorname{smb}($ xcenter_left_200_index,:)) $)$;

xcenter_right_200_index $=$ ceil $\left(\mathrm{PRF}^{*}\right.$ dur/2) +200 ;

[xcenter_right_200_value, xcenter_right_200] =

$\max \left(\left(\operatorname{smb}\left(x c e n t e r \_r i g h t \_200 \_i n d e x,:\right)\right)\right)$;

$\%$ Calculate slopes

left_slope_100 $=($ xcenter - xcenter_left_100 $) / 100$

right_slope_100 $=($ xcenter_right_100 - xcenter $) / 100$

left_slope_200 $=($ xcenter - xcenter_left_200 $) / 200$;

right_slope_200 $=($ xcenter_right_200 - xcenter $) / 200$;

$\%$ Find average slope

avg $=($ left_slope_100+left_slope_200+right_slope_100+right_slope_200)/4; \%

Change in range sample per change in azimuth sample

$\mathrm{x} \_$velocity $=\operatorname{avg} * \mathrm{dx} /(\mathrm{vp} / \mathrm{PRF}) * 100$

end

If the range velocity detection is enabled by setting $\mathrm{x} \_$movement to 1 , the rest of the

algorithm will run. The five points used in the algorithm are first determined by storing

the coordinates at various points on the streak. The points are then used to generate an

average slope value, which is then converted to the approximate range velocity according

to the algorithm. 


\section{Determining Azimuth Velocity with the Range-Compressed Signal}

The algorithm is implemented in code below:

$\%$ EXAMINING AZIMUTH SIGNAL

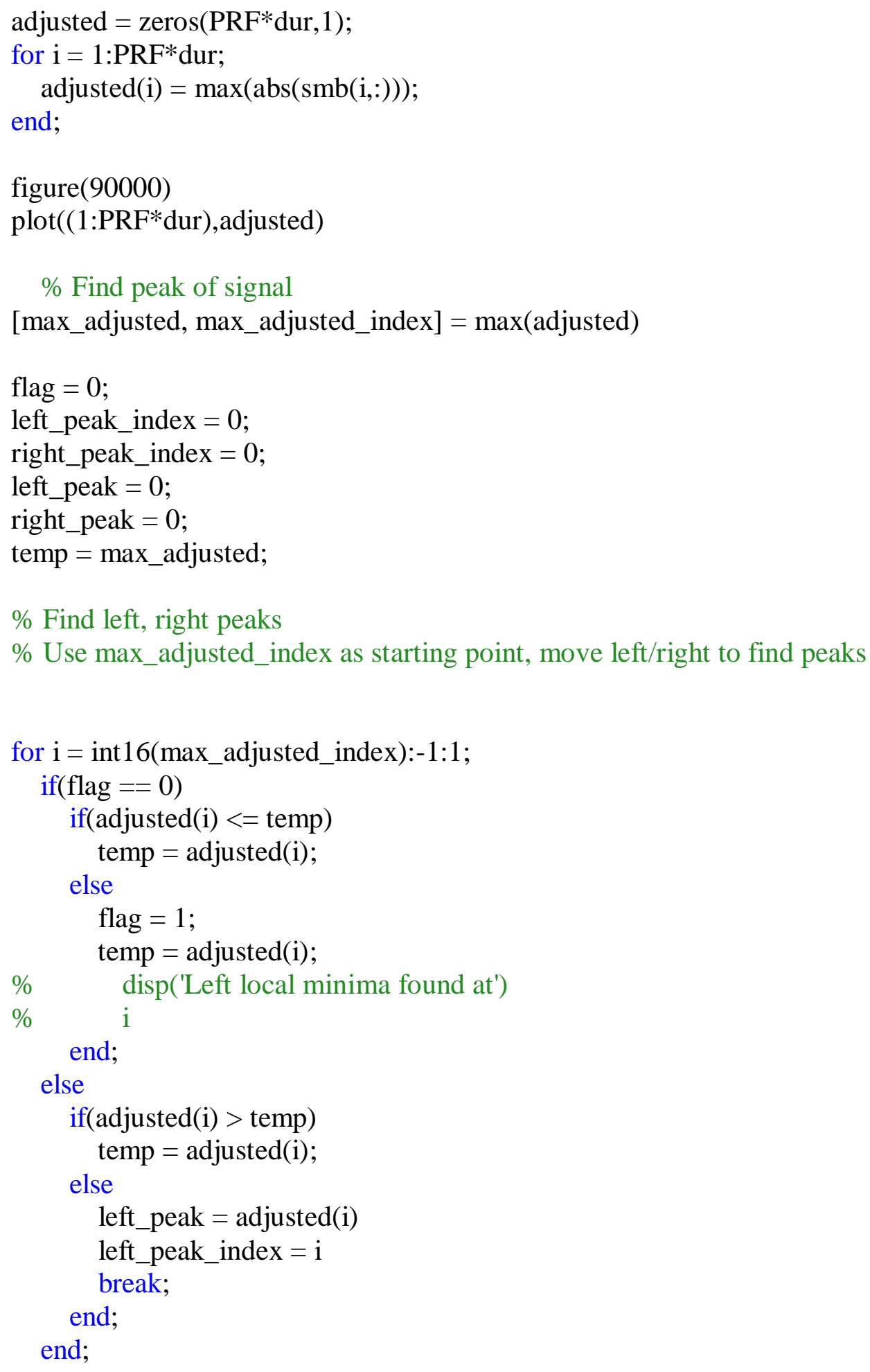


end;

temp $=$ max_adjusted;

flag $=0$;

for $\mathrm{i}=$ int 16(max_adjusted_index):PRF*dur;

if(flag $==0) \quad \%$ Local minima not found yet, expect decreasing values

if(adjusted(i) <= temp) \% left peak not reached yet temp $=$ adjusted $(\mathrm{i})$;

else $\quad \%$ left peak reached, record position and set left flag

flag $=1$;

temp $=$ adjusted(i);

$\% \quad$ disp('Right local minima found at')

$\% \quad$ i

end;

else $\quad \%$ Local minima found, expect increasing values

if(adjusted(i) $>$ temp)

temp $=\operatorname{adjusted}(\mathrm{i})$;

else

right_peak = adjusted(i)

right_peak_index $=\mathrm{i}$

break;

end;

end;

end;

$\%$ Calculate azimuth velocity

if $(\bmod (\operatorname{xn}(1), 3)==0)$

$\operatorname{disp}(' 0$ ')

$\mathrm{x}=$ double $($ max_adjusted_index - left_peak_index $)$;

left_estimate $=-0.0036581 * x^{\wedge} 2+2.55643347 * x-386.6495116$;

$\mathrm{x}=$ double(right_peak_index - max_adjusted_index);

right_estimate $=-0.0036581^{*} \mathrm{x}^{\wedge} 2+2.55643347^{*} \mathrm{x}-386.6495116$;

elseif $(\bmod (x n(1), 3)==1)$

$\operatorname{disp}(' 1$ ')

$\mathrm{x}=$ double(max_adjusted_index - left_peak_index $)$;

left_estimate $=-0.0065613^{*} \mathrm{x}^{\wedge} 2+3.41011545^{*} \mathrm{x}-381.76885$;

$\mathrm{x}=$ double(right_peak_index - max_adjusted_index);

right_estimate $=-0.0065613 * x^{\wedge} 2+3.41011545^{*} \mathrm{x}-381.76885$;

else

$\operatorname{disp}(' 2 ')$

$\mathrm{x}=$ double(max_adjusted_index - left_peak_index $)$;

left_estimate $=-0.0047653 * x^{\wedge} 2+2.96469009 * x-405.68232$;

$\mathrm{x}=$ double(right_peak_index - max_adjusted_index);

right_estimate $=-0.0047653^{*} \mathrm{x}^{\wedge} 2+2.9646900{ }^{*} \mathrm{x}-405.68232$;

end; 


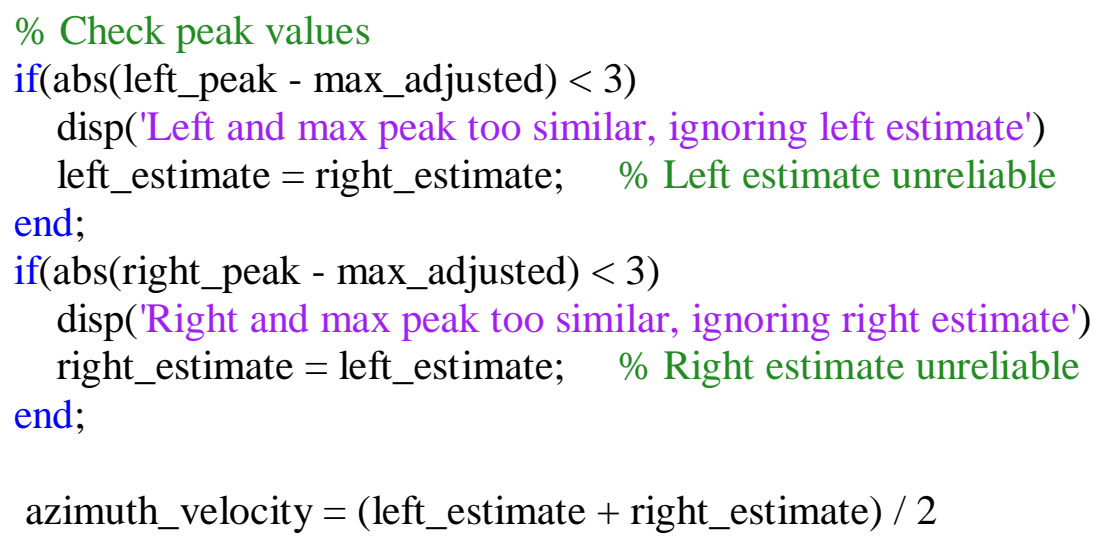

The location of the main lobe is found first, using the $\max$ function in MATLAB. The first two for statements iterate through the waveform and determine the peaks of the two side lobes on either side of the main lobe. Beginning from the index of the main lobe, the algorithm successively checks each point on the waveform for ever-decreasing values. Once a value is found which is larger than the previous value, the local minima is found and a flag is set. Once this occurs, the algorithm now checks for ever-increasing values. A value that is not larger than the previous value is determined to be the local maxima, and that index is considered the position of that side lobe.

Once the locations of both side lobes are determined, the side lobe to main lobe distances are calculated, and the curve-fit equations are applied to calculate the azimuth velocity. The exact equation to be used is dependent on the starting location. The two azimuth velocities are then averaged. If one of the lobes could not be detected correctly, the associated velocity result is disregarded. 


\section{Determining Azimuth Velocity with the Range-Doppler Signal}

Below is the implementation of the Range-Doppler azimuth velocity detection algorithm:

$\%$ Determine Azimuth velocity

if $\left(y \_\right.$movement $\left.==1\right)$

$\%$ Find peak values

$\%$ For testing purposes:

$\%$ xvel $=-60: \mathrm{y}=-100$, index $=385$

$\%$ xvel $=0: \quad \mathrm{y}=0$, index $=518$

$\%$ xvel $=+60: y=+100$, index $=651$

$\%$ Detect azimuth movement, store "peak" locations

flag $=1$;

num_azimuth_target $=0$;

current_max $=0$;

sums = zeros $(1$, ntarget $)$;

azimuth_target $=$ zeros $(1$, ntarget $)$;

mean $=$ zeros $(1$, ntarget $)$;

variance $=$ zeros $(1$, ntarget $)$;

for $\mathrm{k}=1: \mathrm{PRF}^{*} \mathrm{dur}$

$\%$ Keep track if current position is in the trail

$\%$ Use threshold of 5000 to determine location of tracks

if flag == $0 \& \& \operatorname{abs}\left(\mathrm{fsmb}\left(\mathrm{PRF}^{*} \mathrm{dur} / 2, \mathrm{k}\right)\right)>5000$

$\%$ Found a track

flag $=1$;

num_azimuth_target $=$ num_azimuth_target +1 ;

current_max $=\operatorname{abs}\left(\mathrm{fsmb}\left(\mathrm{PRF}^{*} \mathrm{dur} / 2, \mathrm{k}\right)\right)$;

$\% \operatorname{sums}($ num_azimuth_target $)=\operatorname{abs}\left(\mathrm{fsmb}\left(\mathrm{PRF}^{*} \mathrm{dur} / 2, \mathrm{k}\right)\right)$;

azimuth_target(num_azimuth_target) $=\mathrm{k}$;

elseif flag $==1 \& \& \operatorname{abs}\left(\operatorname{fsmb}\left(\mathrm{PRF}^{*}\right.\right.$ dur $\left.\left./ 2, \mathrm{k}\right)\right)>5000$

$\%$ Continuing on a track

if $\operatorname{abs}\left(\operatorname{fsmb}\left(\mathrm{PRF}^{*} \mathrm{dur} / 2, \mathrm{k}\right)\right)>$ current_max

current_max $=\operatorname{abs}\left(\mathrm{fsmb}\left(\mathrm{PRF}^{*} \mathrm{dur} / 2, \mathrm{k}\right)\right)$;

azimuth_target(num_azimuth_target) = k;

end

elseif flag $==1 \& \& \operatorname{abs}\left(\mathrm{fsmb}\left(\mathrm{PRF}^{*}\right.\right.$ dur $\left.\left./ 2, \mathrm{k}\right)\right)<=5000$

$\%$ Left the track, reset flag, variable

flag $=0$;

current_max $=0$;

elseif flag $==0 \& \& \operatorname{abs}\left(\right.$ fsmb $\left(\mathrm{PRF}^{*}\right.$ dur $\left.\left./ 2, \mathrm{k}\right)\right)<=5000$

$\%$ Off the track, do nothing

end

end 


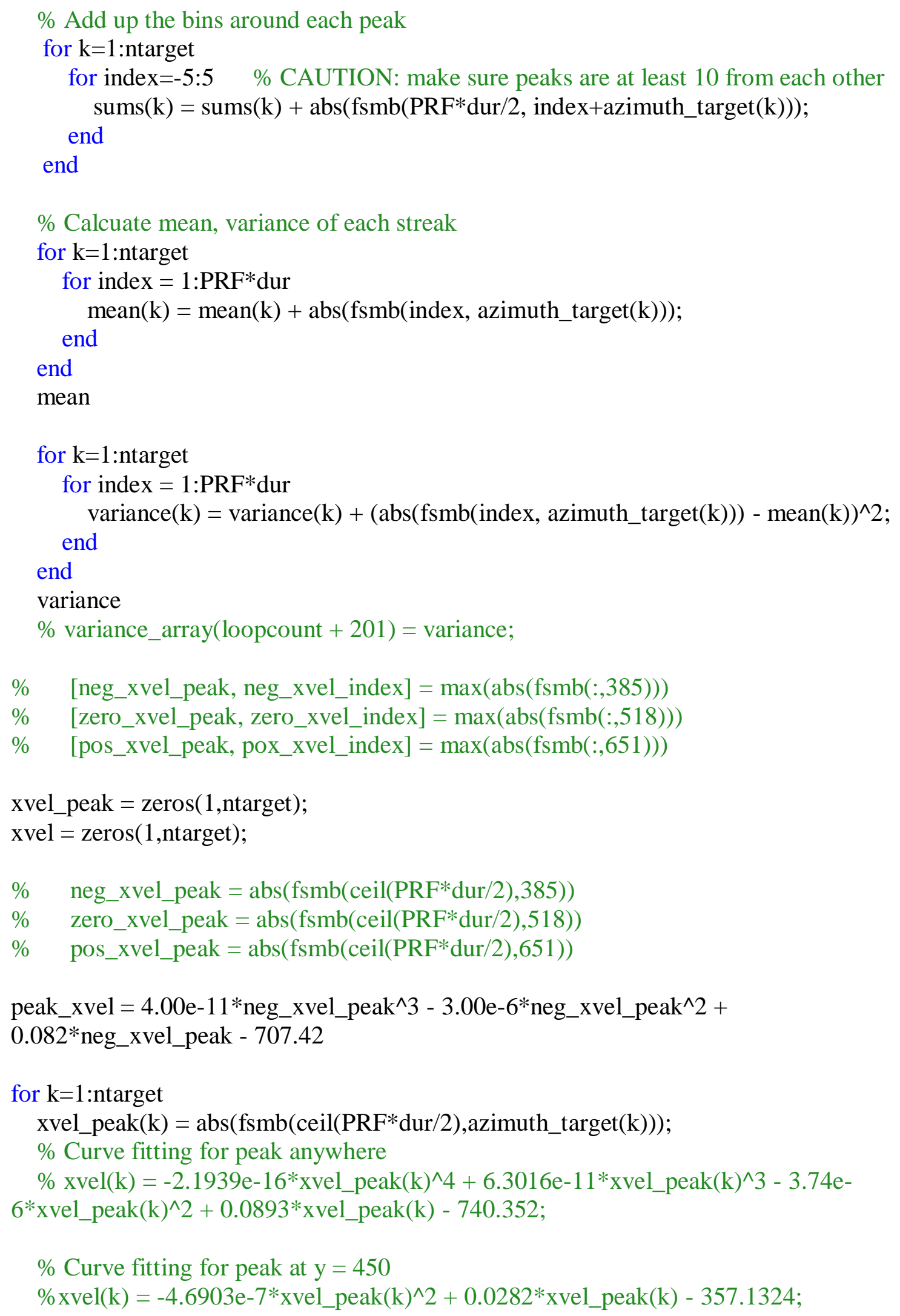




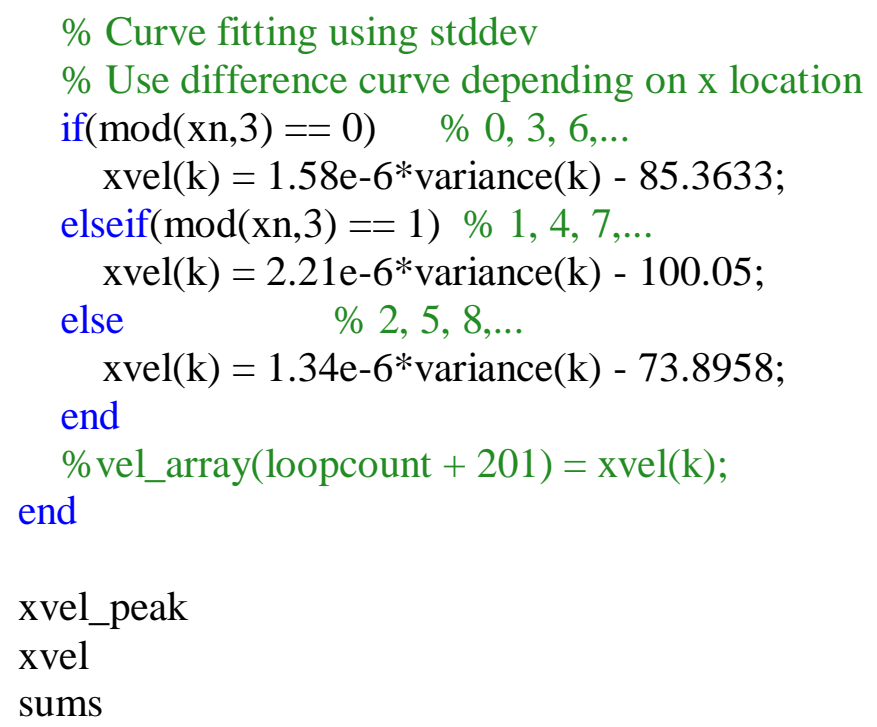

The first for statement scans the Range-Doppler image for signal returns, signifying a target. When one is found, the average and variance of each track is calculated. This data is then used to estimate the azimuth velocity of the target, using the two methods as described in the paper. 